\title{
Design and development of a prototype of dual modality PET/ optical breast cancer margin specification imager
}

\author{
Krishna Nandanavanam \\ West Virginia University
}

Follow this and additional works at: https://researchrepository.wvu.edu/etd

\section{Recommended Citation}

Nandanavanam, Krishna, "Design and development of a prototype of dual modality PET/optical breast cancer margin specification imager" (2010). Graduate Theses, Dissertations, and Problem Reports. 2176. https://researchrepository.wvu.edu/etd/2176

This Thesis is protected by copyright and/or related rights. It has been brought to you by the The Research Repository @ WVU with permission from the rights-holder(s). You are free to use this Thesis in any way that is permitted by the copyright and related rights legislation that applies to your use. For other uses you must obtain permission from the rights-holder(s) directly, unless additional rights are indicated by a Creative Commons license in the record and/ or on the work itself. This Thesis has been accepted for inclusion in WVU Graduate Theses, Dissertations, and Problem Reports collection by an authorized administrator of The Research Repository @ WVU. For more information, please contact researchrepository@mail.wvu.edu. 


\title{
Design and development of a prototype of dual modality PET/optical breast cancer margin specification imager
}

\author{
Krishna Nandanavanam
}

Thesis submitted to the

College of Engineering and Mineral Resources at West Virginia University

in partial fulfillment of the requirements for the degree of

\author{
Master of Science \\ in \\ Mechanical Engineering \\ Larry E. Banta, Ph.D., Chair \\ Sam Mukdadi, Ph.D. \\ Stanislaw Majewski, Ph.D. \\ Department of Mechanical and Aerospace Engineering \\ Morgantown, West Virginia \\ 2010
}

Keywords: Breast Cancer Imager, 3D Reconstruction, Image Processing 


\section{Abstract \\ Design and Development of a Prototype of Dual Modality PET/Optical Breast Cancer Margin Specification Imager}

\section{Krishna Nandanavanam}

This thesis is an attempt to develop a prototype of a breast cancer imager to assess the margins of excised breast tissue. This device is proposed to be used to assure that the entire cancerous lesion has been removed by imaging the intensity of a radio labeled tracer and inspecting for a noncancerous margin in the volume of the tissue sample. The dual modality PET/optical breast cancer specification imager uses a pair of small PET detectors to measure the bioactivity of an excised sample of tissue. A co-registered optical image is taken to allow the surgeon to visually correlate the position of the lesion images obtained with the PET imager within the physical limits of the specimen, and to verify if margins are sufficient. This assists the surgeon in deciding if additional tissue removal is required. The hardware and software required to operate the optical modality was designed and developed as a part of this thesis. Various phantoms were tested and it was demonstrated that 3D reconstruction of volume can be calculated for objects with perfect convex surfaces and irregular convex surfaces of different sizes using the optical modality. By performing error analysis it was found out that the 3D surface can be reconstructed with an accuracy of \pm 1 $\mathrm{mm}$. A calibration procedure was followed to merge the PET and optical volumes. Fusion of PET and optical volumes and margin evaluation of the merged volume was successful. It is proved that dual modality imager can be used for margin analysis and is potential enough for further research in this direction. 


\section{Dedication}

To my beloved parents Sri Latha and Mohan Rao... 


\section{Acknowledgements}

I take this opportunity to appreciate all the individuals who have been with me throughout this journey and have helped me to successfully complete my thesis. I am greatly thankful to Dr. Larry E. Banta for his constant support, guidance and being my research advisor. I am also grateful to Dr. Stanislaw Majewski for his financial support to the project and being my committee member. I extend my thanks to Dr. Sam Mukdadi for his valuable suggestions and for being on my committee. I thank my team Dr. Alexander Stolin and Pete Martone for their valuable suggestions.

Additionally, I would like to deeply thank my family for their constant support and patience. I also thank my friend Shadik Ali for being with me and believing in me that I can accomplish the job. And lastly thanks to all my awesome friends in Morgantown Jyothi, Raghu, Savan, Kartheek, Spoorthi, Mohita, Jagadish, Srikanth and Eswar for making my graduate experience wonderful. 


\section{TABLE OF CONTENTS}

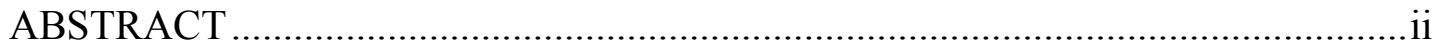

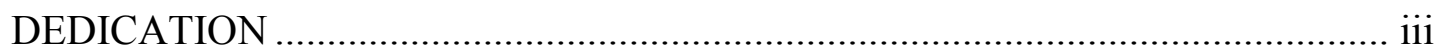

ACKNOWLEDGEMENTS ............................................................................ iv

LIST OF FIGURES............................................................................... viii

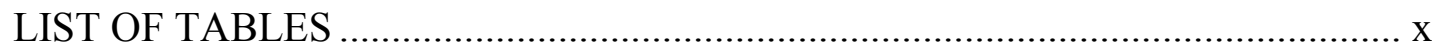

1 GENERAL INTRODUCTION .............................................................. 1

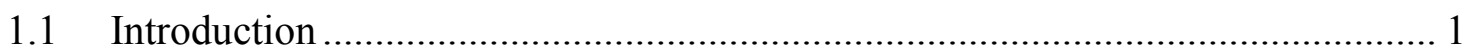

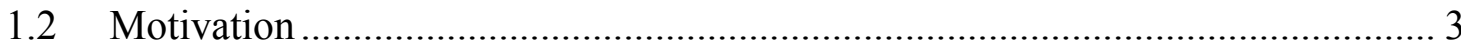

1.3 Proposed Novel Approach ......................................................................... 4

1.4 Objectives and Contributions ................................................................ 5

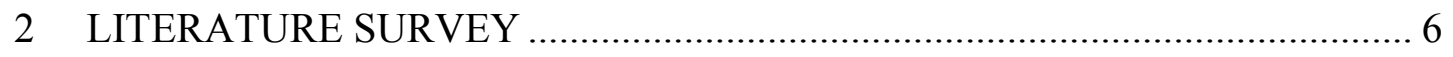

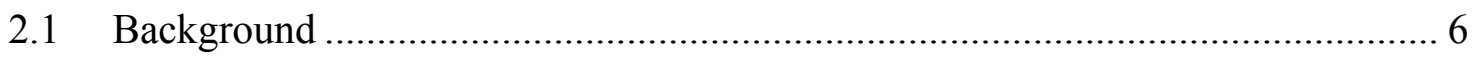

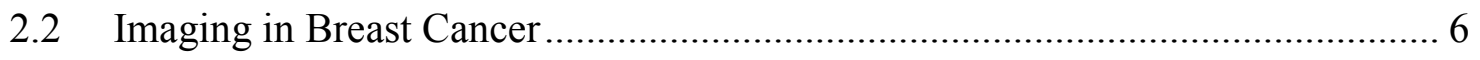

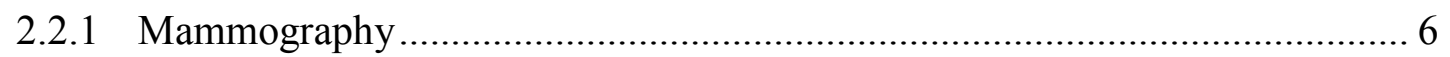

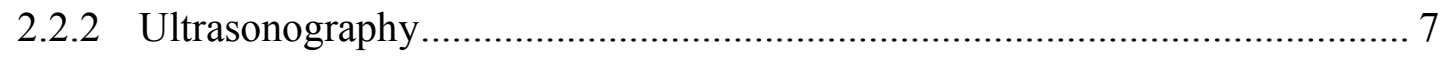

2.2.3 Magnetic Resonance Imaging ........................................................ 7

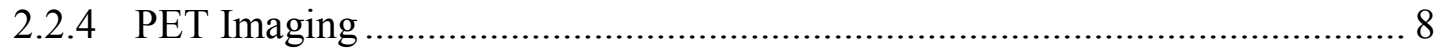

2.3 Justification of PET .............................................................................. 13

2.4 Margin Assessment Methods .............................................................. 14

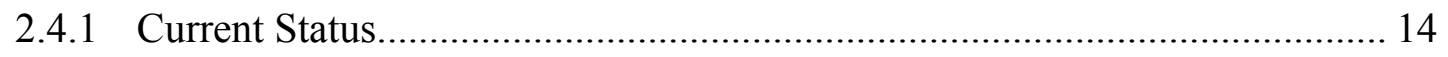

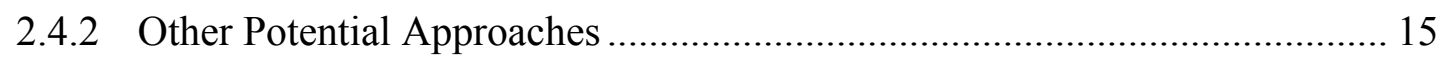

3 MATERIALS AND METHODS ................................................................ 17

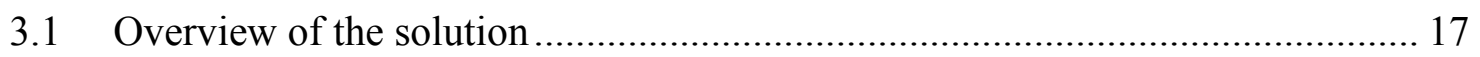




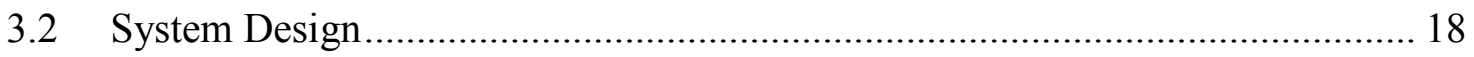

3.2.1 Key components of the Dual Modality PET/Optical Imager.......................... 18

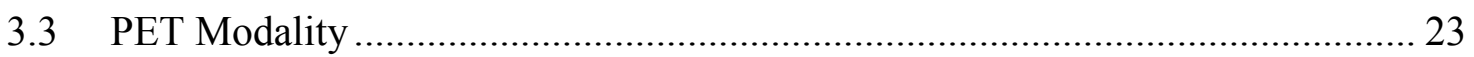

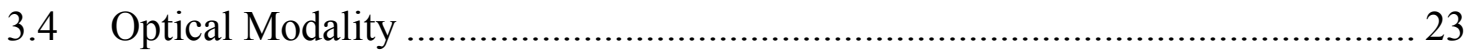

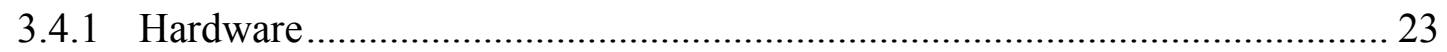

3.4.2 Technical Approach, Optical 3D Reconstruction ......................................... 24

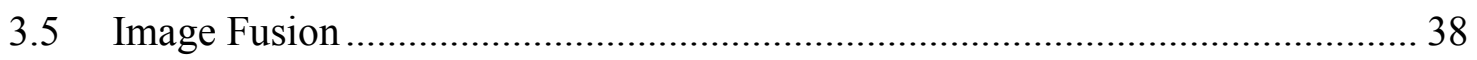

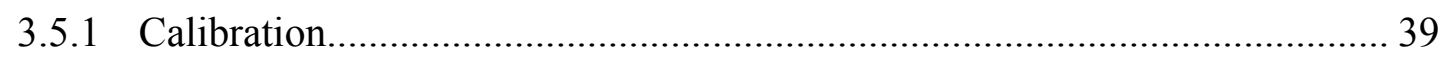

3.5.2 Homogeneous Coordinate Transformation..................................................... 43

3.5.3 Image Fusion with Phantom …………………………………................ 50

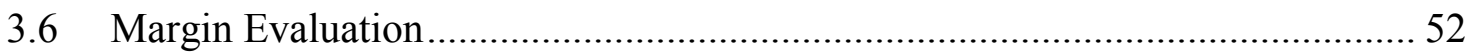

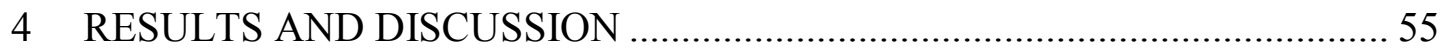

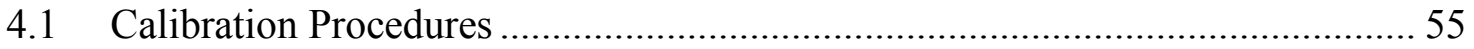

4.1.1 Pixel to Millimeter Calibration ................................................................... 55

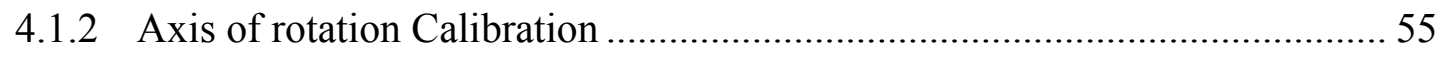

4.1.3 Calibration for Image Fusion ................................................................. 55

4.2 Optical 3D Reconstruction and Slice Generation .............................................. 57

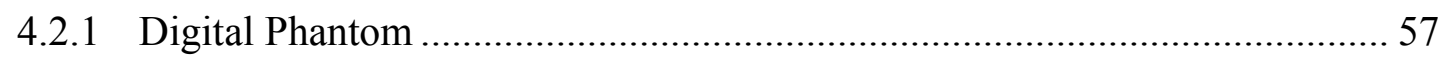

4.2.2 Perfect Convex object: Squash Ball........................................................ 59

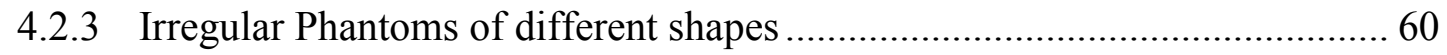

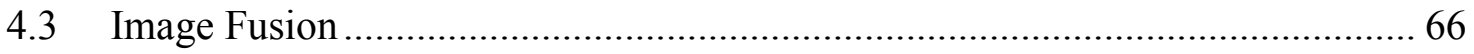

4.3.1 Image Fusion with Phantom ....................................................................... 66

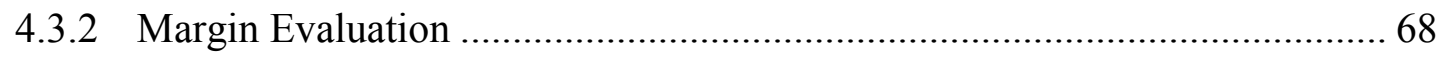

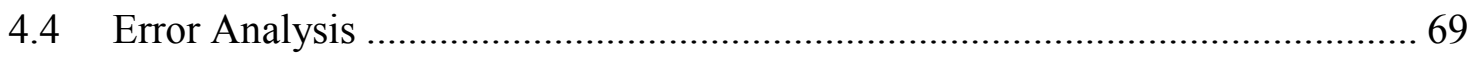

5 CONCLUSIONS AND FUTURE STUDY …………................................... 72 
5.1 Conclusion from the Experimental Results................................................ 72

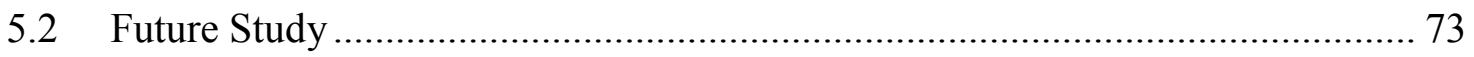

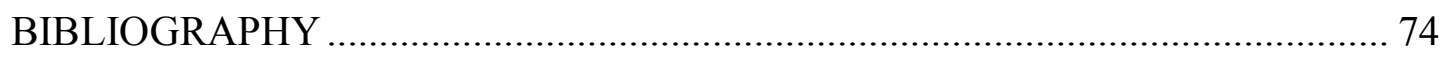

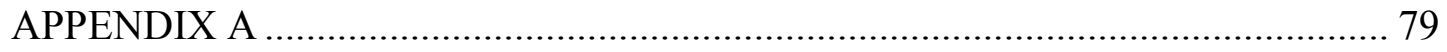

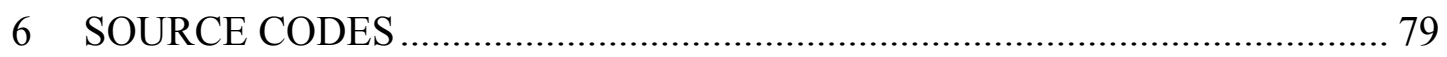

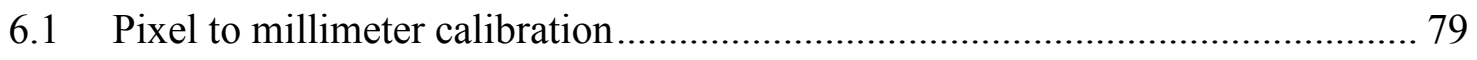

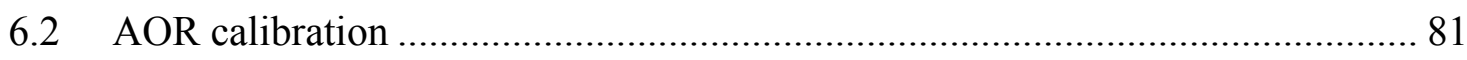

6.3 Image Fusion Calibration ....................................................................... 83

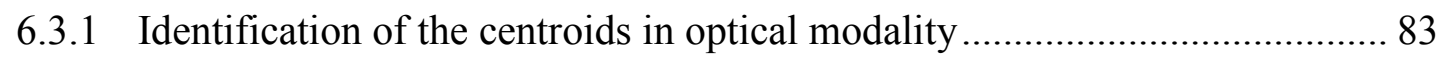

6.3.2 Identification of the centroids in PET modality......................................... 95

6.3.3 Homogeneous transformation Matrix ................................................... 98

6.4 Optical Slice Generation, Merging and Margin Evaluation........................... 100

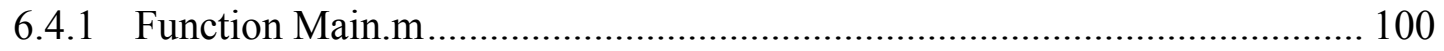

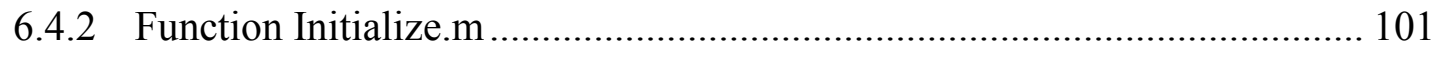

6.4.3 Function Optical_Coordinates.m ......................................................... 103

6.4.4 Function Pet_Coordinates.m.............................................................. 106

6.4.5 Function Slice_Generation.m .......................................................... 108

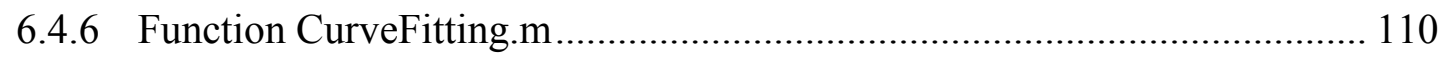

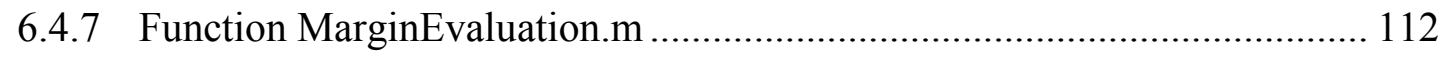




\section{List of Figures}

Figure 1: PET scan showing locally advanced right breast cancer with axillary metastasis

Figure 2: FDG-PET detecting liver metastasis.. ............................................ 12

Figure 3: Images obtained with the portable gamma camera. ............................... 16

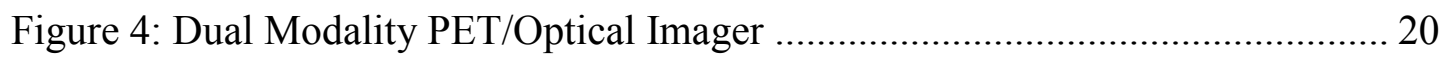

Figure 5: Schematic Diagram of the Top View of the PET/ Optical Imager............. 21

Figure 6: (a) Rotary Table (b) Smart Motor........................................................... 21

Figure 7: Components of the PET/Optical imager................................................. 22

Figure 8: Images of the PET/Optical Imager. (a) Front View (b) Top View (c) Back

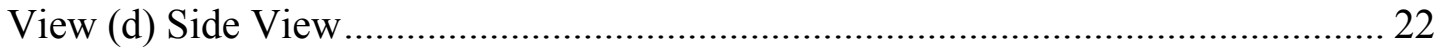

Figure 9: Top view of the DMI showing the object coordinate system.................... 24

Figure 10: Multiple view silhouettes of the specimen taken at different angles........ 25

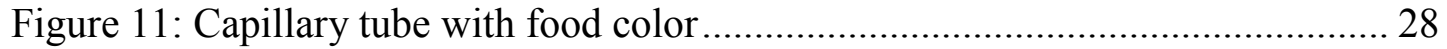

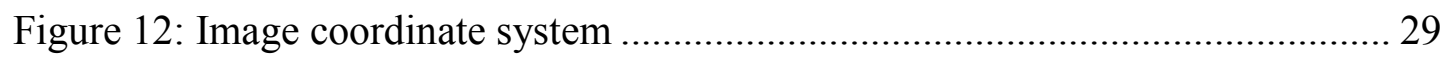

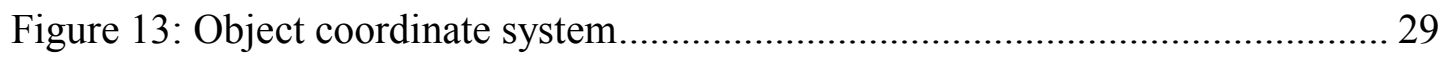

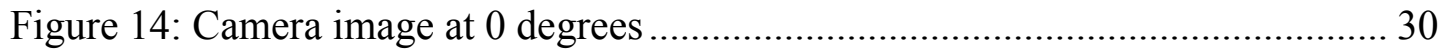

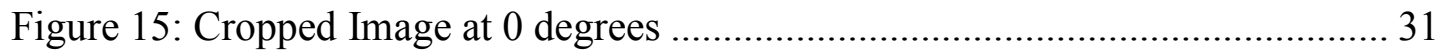

Figure 16: Edges identified for images taken at different angles........................... 32

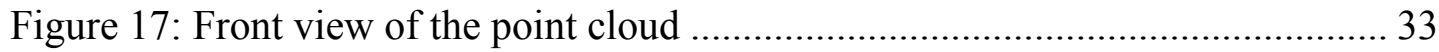

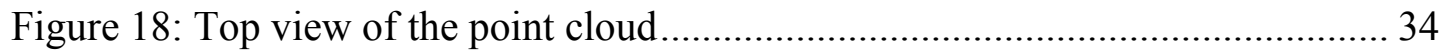

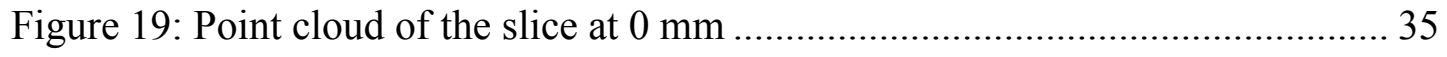

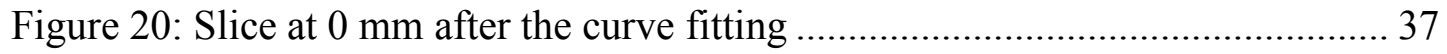

Figure 21: Image of the slice at $0 \mathrm{~mm}$ after the curve fitting ................................ 38

Figure 22: Calibration Phantom for Image Fusion .......................................... 40

Figure 23: Edges of the four spheres identified in the reference image.................... 41

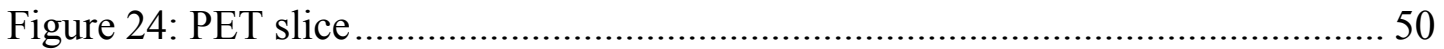

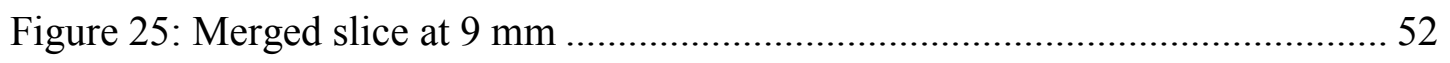

Figure 26: Margins in an excised breast tissue ............................................... 53

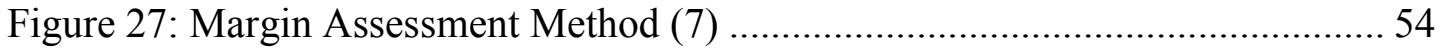

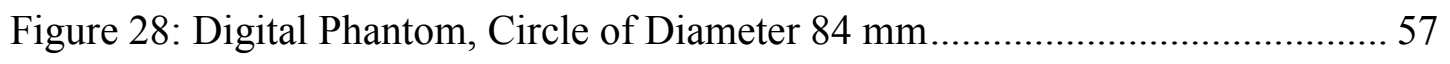


Figure 29: Reconstructed sphere from the point cloud generated from the camera

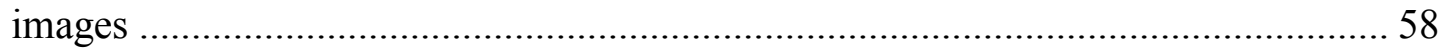

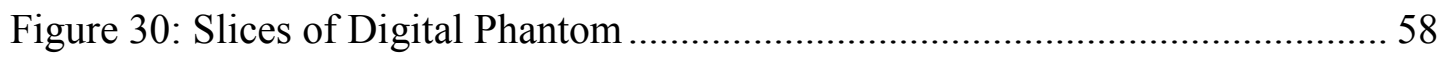

Figure 31: Double Yellow Squash Ball ................................................................ 59

Figure 32: Slices of Squash Ball ........................................................................ 59

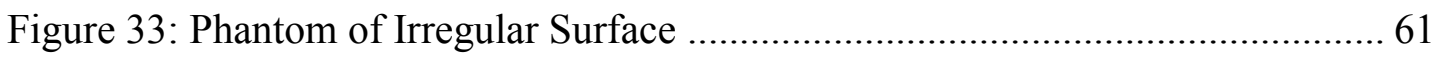

Figure 34: 3D surface plot of the reconstructed Point Cloud........................................ 61

Figure 35: Slices of Irregular Phantom Shown in Figure 31 ...................................... 62

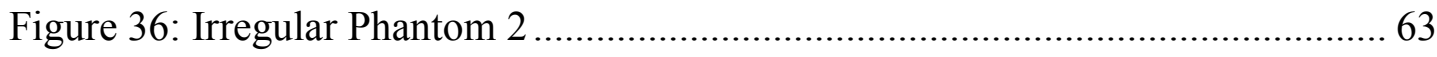

Figure 37: 2D Plot of the 3D reconstructed point Cloud of Phantom 2..................... 63

Figure 38: Slices of Phantom 2 with Irregular Surface shown in Figure 34 .............. 64

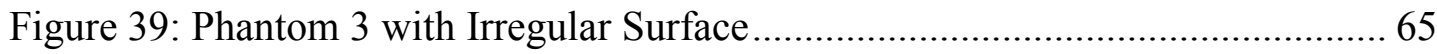

Figure 40: 2D Plot of 3D Reconstructed Point Cloud of Phantom 3 ......................... 65

Figure 41: Slice of Phantom 3 with Irregular Surface shown in Figure 37 ............... 66

Figure 42: Phantom used for Image Fusion ............................................................... 67

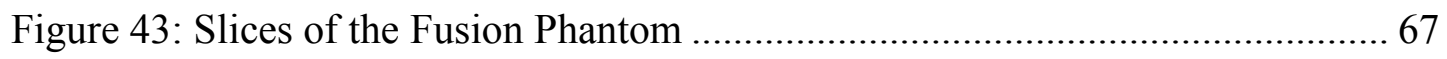

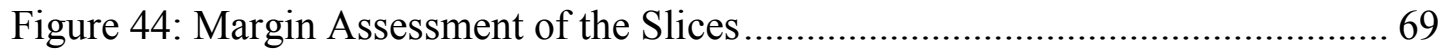

Figure 45: Error analysis based on degree of rotation ............................................. 70

Figure 46: Angle of Rotation versus Total time and Percentage Error ....................... 71 


\section{List of Tables}

Table 1: Axillary Lymph Node Staging in Patients with Early Stage Breast Cancer

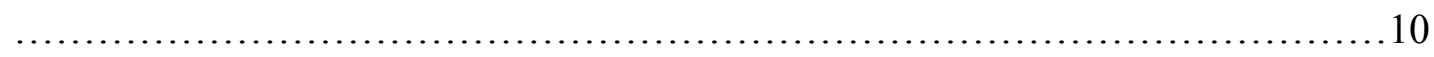

Table 2: Use of PET in Breast Cancer Patients with Distant Metastasis. (16). ......... 12

Table 3: Acceptable margins for DCIS and invasive carcinoma by surgeons (33) ... 14

Table 4: $(\mathrm{X}, \mathrm{Y}, \mathrm{Z})$ coordinates of the centroids of spheres in optical and PET

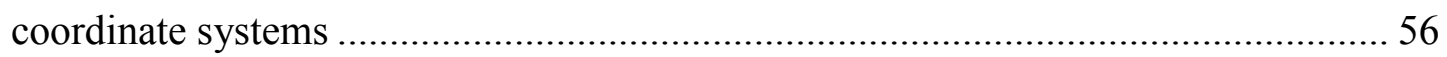

Table 5: Error Analysis for the intervals of Degree of Rotation.............................. 70 


\section{General Introduction}

\subsection{Introduction}

Breast Conservation Therapy has become a much sought alternative to mastectomy in the treatment of smaller cancerous lesions as both the procedures are equally effective. Lumpectomy is a breast conserving technique where in the surgeon removes the cancerous tumor from the breast surrounded by healthy tissue. Major clinical trials have demonstrated similar survival rates for stage II breast cancer patients undergoing breast conservation therapy (lumpectomy followed by radiation therapy) and mastectomy (1). Also there is no increased risk of developing second malignancies in patients undergoing lumpectomies compared with mastectomy (2).

There have been huge advances in the technologies used for breast cancer imaging. Mammography has been the gold standard in identifying breast cancer due to the factors associated with performance, reliability and cost effectiveness. Mammogram uses X-rays to examine and is the most effective technology available for breast cancer diagnosis. Constant effort is being made to provide more information to the surgeon about the lesion localization, to decrease the intensity of radiation used in mammography; to improve the image quality and to develop techniques to interpret these images. Digital Mammography increases the sensitivity and optimizes the contrast between the malign and benign tissues. Digital Mammographic Imaging Screening Trials (DMIST) conclude that more accurate detection of cancer is possible with digital mammography for pre- or perimenopausal women under the age of 50 or women of any age with dense breasts. Patients are exposed to reduced doses of radiation in digital mammograms than film mammograms (3).

Computer-aided Detection (CAD) systems may be used to improve the accuracy of conventional and digital mammograms. Ultrasonography is an adjunct technology to mammography which can be used in improving the overall sensitivity of the system. This is painless and involves no radiation but the results obtained are not consistent in many cases as it depends mainly on the operator's skill(4). The usage of ultrasound in breast diagnosis is still under investigation. Magnetic Resonance Imaging (MRI) is used to determine the extent of cancer in the body after diagnosis. According to the American Cancer Society MRI is 
used for diagnosing patients who have more than $20 \%$ life time risk of developing cancer. MRI may also be useful in imaging dense breast tissues (3).

However mammogram images can be ambiguous in many cases. This makes it difficult to localize the presence of the tumor and might lead to complete resection of the breast. Positron Emission Tomography (PET) is an effective solution in this direction. It has the potential to identify the presence of cancer even when the other imaging techniques give normal results. It is especially helpful in the cases where a mammogram is not able to provide clear information. Advances in nuclear medicine have introduced new techniques like radionuclide imaging. F-18 Flouro-deoxyglucose (FDG) is the most widely used radiopharmaceutical for imaging breast cancer. The cancer cells demonstrate increased metabolic rate compared to normal tissue, and the FDG can be imaged using a PET imager (5).

Once the location of the cancerous tissue is identified the surgeon removes the cancerous lesion under the guidance of the mammogram images. The excised breast specimen is delivered to pathology with the edges of the specimen marked. The marked edges of the specimen are called margins. The success of the surgery lies in achieving negative margins which means adequate healthy tissue is surrounding the cancer cells. If the margins are positive then re-excision is to be done and this entire process may extend up to an hour. Through this entire period of time the patient is held under anesthesia with an open incision. In as many as $25-50 \%$ of cases a follow up surgery is necessary to remove residual cancerous tissue left during the first procedure.

The 'Dual Modality PET/optical Imager' is an attempt to determine these margins of the healthy tissue around the cancerous lesion thereby assisting the surgeon in achieving negative margins. This may potentially avoid the necessity of an additional surgery to remove the cancerous lesions and reduce the waiting time. 


\subsection{Motivation}

The challenge associated with breast conservation procedure in breast cancer treatment is adequate margins while preserving the healthy tissue as much as possible. In most of the cases after the cancerous lesion is removed surgically the excised tissue is sent to the pathology for margin assessment. This process might take 20-30 minutes or more to get a confirmation that adequate margins are achieved. The surgeon and the patient must wait to hear from pathology. The waiting time may further be prolonged if adequate margins are not achieved as expected and this process may be repeated several times before the surgery is concluded. Through this entire process the patient is held with an open incision and exposed to anesthesia which can result in increased morbidity and adverse neurological effects of anesthesia. Extended waiting time means an increase in the cost of the surgical procedure to the patients.

Various reports say that positive margins occur in $20-70 \%$ of the patients leading to a second surgery (6). Positive margins can lead to multiple re-excisions which mean additional costs and anxiety to the patients, a compromise in the cosmetic outcome or can possibly result in mastectomy. The benefits associated with the decrease in the rate of positive margins have encouraged many studies to investigate and find a solution to this problem with margin analysis being a step in that direction. Surgical margin analysis has proved to be a critical component of breast conservation therapy in spite of various issues being controversial (6). Intra-operative margin assessment has been investigated as a tool to reduce the rate of positive margins as reexcision is done at the same time, thereby eliminating the need for a second surgery. This importance raises a need to find a standardized procedure to calculate the margins and to help the patient by reducing the need for multiple re-excisions and also help the surgeon in making treatment decisions. The margin assessment methods may influence the final pathological results and ultimately the treatment decisions taken by the surgeons (7).

Various margin analysis techniques like frozen section analysis, permanent section analysis and multicolor inking systems have been studied. While all these techniques may reduce the rate of positive margins, the problem still persists. Reports show that at least $20 \%$ of the patients return to the operating room for re-excision. Despite the best efforts of the surgeons the rates of re-excisions continue to be high (4). From these numbers it is understood that there is a dire need to investigate new technologies which can reduce the rates of positive margins. 


\subsection{Proposed Novel Approach}

The dual modality PET /Optical imager (DMI) is a table top or mobile $3 \mathrm{~d}$ imaging system, located in the operating room (OR), and providing fast in-situ determination of tumor resection and the adequacy of surgical margins. DMI uses PET and optical modalities to evaluate the margins of an excised sample of tissue, typically a cancerous lesion surgically removed from a patient, with an appropriate healthy tissue margin. The Positron Emission Tomography (PET) Tissue Sample system uses a pair of small PET detectors to identify the cancerous lesion by imaging the intensity of a radio labeled tracer in the excised breast tissue while the optical modality uses a camera to give the physical outline of the same.

The excised specimen is placed on a specimen holder which is present on a rotary table. For the optical reconstruction the specimen is rotated from 0 to 350 degrees and an image is captured by the camera every $10^{\circ}$. PET images are taken at the same time. Optical 3D reconstruction of the surface of the excised breast tissue is done from the 36 projections taken by the camera while the PET process produces images of only the cancerous tissue. 3D volumes obtained from both the modalities are merged and the margins are evaluated. If there are any positive margins the surgeon can go back to the patient and re-excise to obtain negative margins. The processing time of the imager for margin assessment is anticipated to be 15 minutes.

Such an instrument can reduce the overall surgery time and the number of iterations required for adequate margins although the final margin confirmation may have to be obtained from the pathology. The DMI may also reduce the number of follow-up surgeries required. 


\subsection{Objectives and Contributions}

The objective of this research is to develop the optical modality of the prototype of a dual modality PET/Optical imager and perform the margin assessment by merging the reconstructed PET and optical images. The scope of the work is as follows:

- Add the optical modality to the existing PET prototype.

- Develop software to do the 3D reconstruction and slice generation of the surface of the excised specimen from the optical data.

- Mathematically merge the PET and optical volumes.

- Evaluate the margins of the merged volume to determine the adequacy of the margins obtained.

- Develop calibration fixtures and procedures for the DMI.

- Test and demonstrate the system. 


\section{Literature Survey}

\subsection{Background}

Breast cancer is the second major cause of deaths due to cancer in women, after lung cancer and more than 184,000 patients were diagnosed with breast cancer in 2008. In 2008 more than 40,480 women died from breast cancer in the USA. Breast cancer can be treated if it is diagnosed early. More than $90 \%$ of the patients have localized disease during diagnosis in western countries. One in eight of the North American women (who live up to the age of 85) have a lifetime risk of developing breast cancer(3).

\subsection{Imaging in Breast Cancer}

Technological development in various imaging modalities has contributed to advances in breast cancer management and has led to a decrease in the mortality rate due to breast cancer. New evolving diagnostic technologies look promising in detecting cancer at an early stage and in improving the breast cancer survival rate. Early detection of cancer is the key factor in increasing the survival rate from breast cancer.

\subsubsection{Mammography}

Mammographic screening helps in early diagnosis and has contributed to a $25 \%$ to $30 \%$ decrease in mortality rate (3). It has been the gold standard for breast cancer detection and is found to be the best method to decrease the mortality rate due to breast cancer. Accuracy of mammography is influenced by factors like age and dense breasts. Sensitivity varies from $62.9 \%$ in women with extremely dense breasts to $87.0 \%$ in women with almost entirely fatty breasts (8). In the case of dense breasts the sensitivity is as low as 30-48\%(9). Sensitivity is also influenced by age and increases from $68.6 \%$ in women 40 to 44 years of age to $83.3 \%$ in women 80 to 89 years of age (8). These factors along with a family history of breast cancer and long intervals between mammograms may lead to false positive results in mammography. Mammography has limitations in imaging dense and augmented breasts and plays a very limited role in axillary lymph node metastasis. 


\subsubsection{Ultrasonography}

Screening mammography is the best available technique for breast cancer detection, yet some breast cancers cannot be identified by mammography. Ultrasound is an adjunct technique to mammography for breast cancer diagnosis. Ultrasonography (US) can be used for additional information when mammograms are ambiguous. This technique is especially helpful in diagnosing young, pregnant and lactating women, women with dense breasts and those at a high risk of developing cancer. US may detect 3-4 cancers additionally compared to mammography per 1000 women in the high risk category(10). The role of US in breast cancer detection is still being studied in a large randomized trial, conducted by the American College of Radiologists Imaging Network (ACRIN). Use of US requires highly experienced operators but the benefits are that the process is painless and requires no radiation (11).

\subsubsection{Magnetic Resonance Imaging}

Various studies conducted from the mid to late 1990s reported significantly higher sensitivity in breast cancer diagnosis for MRI compared with the other modalities. American Cancer Society guidelines recommend MRI screening as an adjunct to mammography for women who have a lifetime risk of $20-25 \%$ or greater. It is demonstrated by several studies that MRI has higher sensitivity in determining small tumors compared with mammography (12). The exceptional ability of MRI in detecting cancers and in identifying small tumors compared to other modalities is the reason for the increasing popularity of this technology as a supplement in breast cancer diagnosis. MRI screening is limited only for the patients with high risk of breast cancer because of its high cost and the requirement for intravenous contrast injection. Claustrophobia also reduces the tolerance of the patients to imaging process(13). 


\subsubsection{PET Imaging}

Positron Emission Tomography is a radionuclide imaging technique used in management of various malignancies and images the tissue based on its metabolic activity. It was initially developed to observe the functional changes in the brain by looking at the brain's biochemical activities. Lately PET is being used to diagnose various malignancies. Pet imaging is done by injecting the patient with a radionuclide such as F-18 Flouro-deoxyglucose, a radiopharmaceutical that is preferentially metabolized by cancer cells. The F-18 nuclei are proton rich and emit positrons which have a short life. After travelling a few millimeters positrons $(\beta+)$ interact with tissue electrons ( $\beta$-) resulting in two $511 \mathrm{KeV}$ annihilation photons which are released at $180^{\circ}$ to one another. These gamma photons are then detected by the PET cameras (14).

Malignant tissue has a higher metabolic rate compared to the adjacent healthy tissue and this is targeted by PET using a radiopharmaceutical like 2-[18F] flouro-2-deoxy-D-glucose (FDG) which is glucose analog. FDG- PET works by providing functional information about the tissue compared with the anatomic details given by the other modalities. PET- FDG is used to detect malignancies in the lung, brain, head and neck, colon and breast. PET has high sensitivity to detect breast cancer(15). FDG is widely used because breast cancer is avid of FDG and it has a comparatively long physical life. FDG-PET is found to be effective in patients who have ambiguous mammographies(5).

A study conducted by Noh D-Y et al. for determining the diagnostic value of PET in detecting breast cancer reported that PET has high sensitivity for breast cancer and can be used as an accurate diagnostic tool. PET displayed excellent diagnostic accuracy in detecting primary tumor mass $(97 \%)$ in comparison with physical examination (78\%) and mammography (67\%). As a part of this study 27 patients have undergone breast operations based on PET results from June 1995 to November 1996. For axillary lymph node metastasis PET had a 96\% accuracy compared with physical examination (74\%) and mammography (60\%) (15) . 
PET has a promising role in detecting distant metastasis and recurrence of breast cancer. PET is also proved to be helpful in prognosis and in understanding a patient's response to the treatment. This would help to make a decision on the treatment methods to be followed for the patient. The role of PET in breast cancer management is briefly discussed in the following sections.

\subsubsection{Early Stage Breast Cancer Diagnosis}

The role of FDG-PET in determining breast cancer in early stages is limited due to its limited ability to identify small lesions and the high costs associated(16). In one of the studies conducted by Avril et al it was observed that zero were detected out of four tumors with size less than $0.5 \mathrm{~cm}$ while one out of eight tumors was identified for tumors with sizes of $0.5 \mathrm{~cm}$ to 1.0 $\mathrm{cm}$. Diagnostic accuracy of FDG-PET increased with the tumor size from $68.2 \%$ (tumor size $\leq$ $2.0 \mathrm{~cm}$ ) to $91.9 \%$ (tumor size $>2-5.0 \mathrm{~cm}$ ) and reached $100 \%$ for tumors with size greater than 5 $\mathrm{cm}(17)$.

\subsubsection{Breast Positron Emission Mammography}

Positron Emission Mammography (PEM) is a technique where PET results are integrated with the results obtained from conventional mammography. PEM can detect smaller lesions unlike whole body PET and provides better visualization of dense breasts and breasts with fibrocystic disease (16). 


\subsubsection{Axillary Lymph Node Staging}

Axillary lymph node involvement is an important factor which affects the treatment selection and prognosis at localized level. Nuclear medicine technologies have gained popularity in detecting axillary lymph node involvement due to limitations of non nuclear methods. It is reported that FDG-PET gives equally good results as sentinel lymph node (SLN) biopsy in identifying the status of lymph nodes when the surgery is performed by a well trained surgeon $(18)(15)(19)$. It is seen from Table 1 that the sensitivity of PET in detecting axillary node involvement varies widely from $25 \%$ to $90 \%$ while the specificity is around $80 \%$ to $100 \%$. The role of FDG-PET in axillary lymph node staging seems to be increasing but axillary lymph node dissection is followed as a standard procedure at this point due to its better accuracy and sensitivity (16).

Table 1: Axillary Lymph Node Staging in Patients with Early Stage Breast Cancer (16)

\begin{tabular}{|l|c|c|c|l|}
\hline \multicolumn{1}{|c|}{ Study } & N & Sensitivity & Specificity & Author's Conclusions \\
\hline Wahl et al(20) & 308 & $61 \%$ & $80 \%$ & $\begin{array}{l}\text { Multiple intense foci of } \\
\text { PET uptake are } \\
\text { predictive for nodal } \\
\text { involvement }\end{array}$ \\
\hline $\begin{array}{l}\text { Veronesi et } \\
\text { al(21) }\end{array}$ & 236 & $37 \%$ & $96 \%$ & $\begin{array}{l}\text { PET-positive axilla } \\
\text { should have an axillary } \\
\text { lymph node dissection } \\
\text { for axillary staging }\end{array}$ \\
\hline $\begin{array}{l}\text { Van der hoeven } \\
\text { et al(22) }\end{array}$ & 70 & $25 \%$ & $97 \%$ & $\begin{array}{l}\text { Low sensitivity; uptake } \\
\text { was related to axillary } \\
\text { tumor load }\end{array}$ \\
\hline Avril et al(23) & 51 & $79 \%$ & $96 \%$ & $\begin{array}{l}\text { Better detection of } \\
\text { axillary nodes in } \\
\text { tumors }>2.0 \text { cm }\end{array}$ \\
\hline Chung et al(24) & 51 & $60 \%$ & $100 \%$ & $\begin{array}{l}\text { Patients with an uptake } \\
\text { value }>2.3 \text { had axillary } \\
\text { metastases. }\end{array}$ \\
\hline Smith et al(25) & 50 & $90 \%$ & $97 \%$ & $\begin{array}{l}\text { PET may obviate the } \\
\text { need for axillary } \\
\text { surgery in women with } \\
\text { small primary tumors }\end{array}$ \\
\hline
\end{tabular}




\subsubsection{Distant Metastases}

PET has great potential in detecting distant metastases and recurrence of breast cancer as shown in Figure 1. It is considered to be a powerful imaging modality as the whole body can be diagnosed and staging of the tumor can be done in single procedure. PET plays a promising role in evaluating visceral lesions (liver, lung, distant lymph nodes) and bone metastases (26) (18). FDG-PET is superior to the conventional imaging modalities in tumor localization as it can significantly detect more lesions at different sites in the body with a single examination. It is known from different studies that FDG-PET is successful in diagnosing a significant number of metastases which would have been missed or incorrectly diagnosed by other modalities like $\mathrm{CT} / \mathrm{US} / \mathrm{MRI} /$ bone scintigraph as in Figure 2(5). Different studies confirm that PET has a significant role to play in defining the extent of disease and in making a decision regarding the treatment of patients with advanced breast cancer. Table 2 shows the results from different studies which have been done to evaluate the role of PET in detecting distant metastasis.

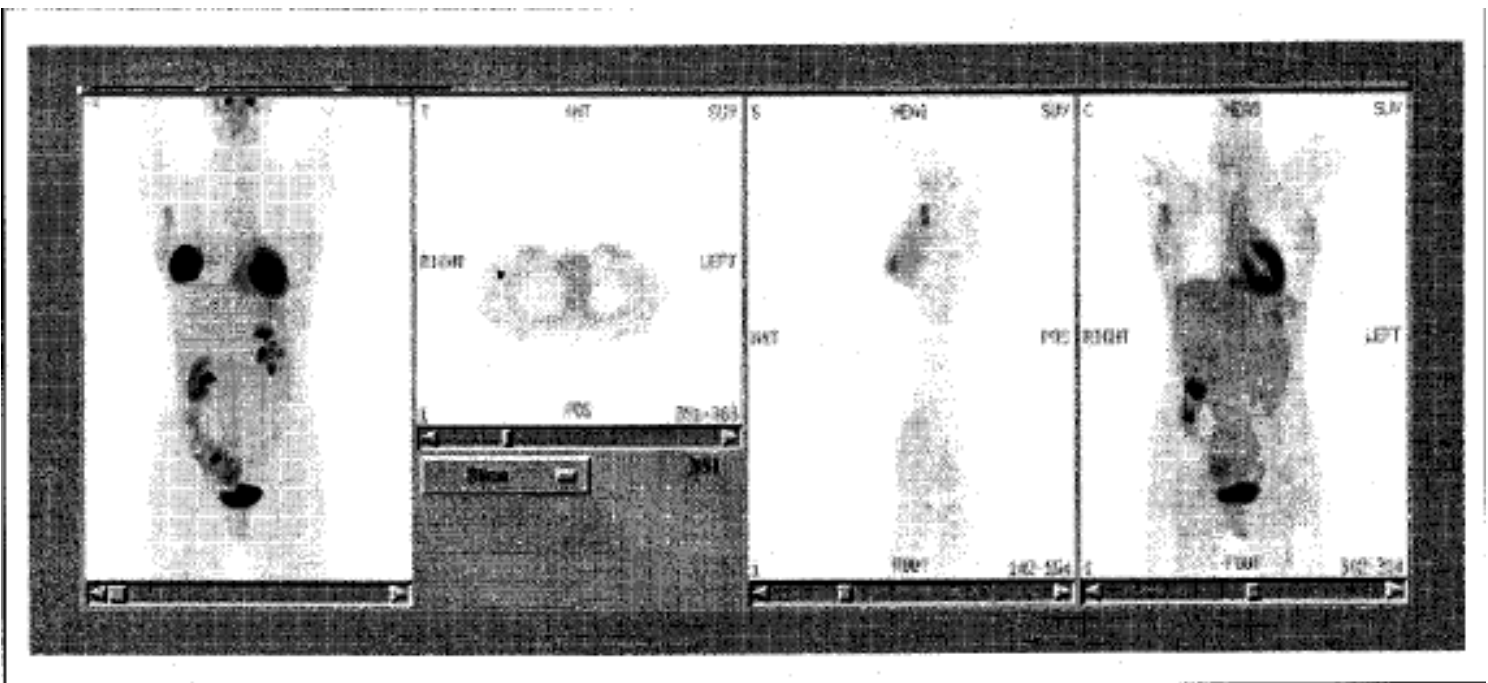

Figure 1: PET scan showing locally advanced right breast cancer with axillary metastasis (11) 


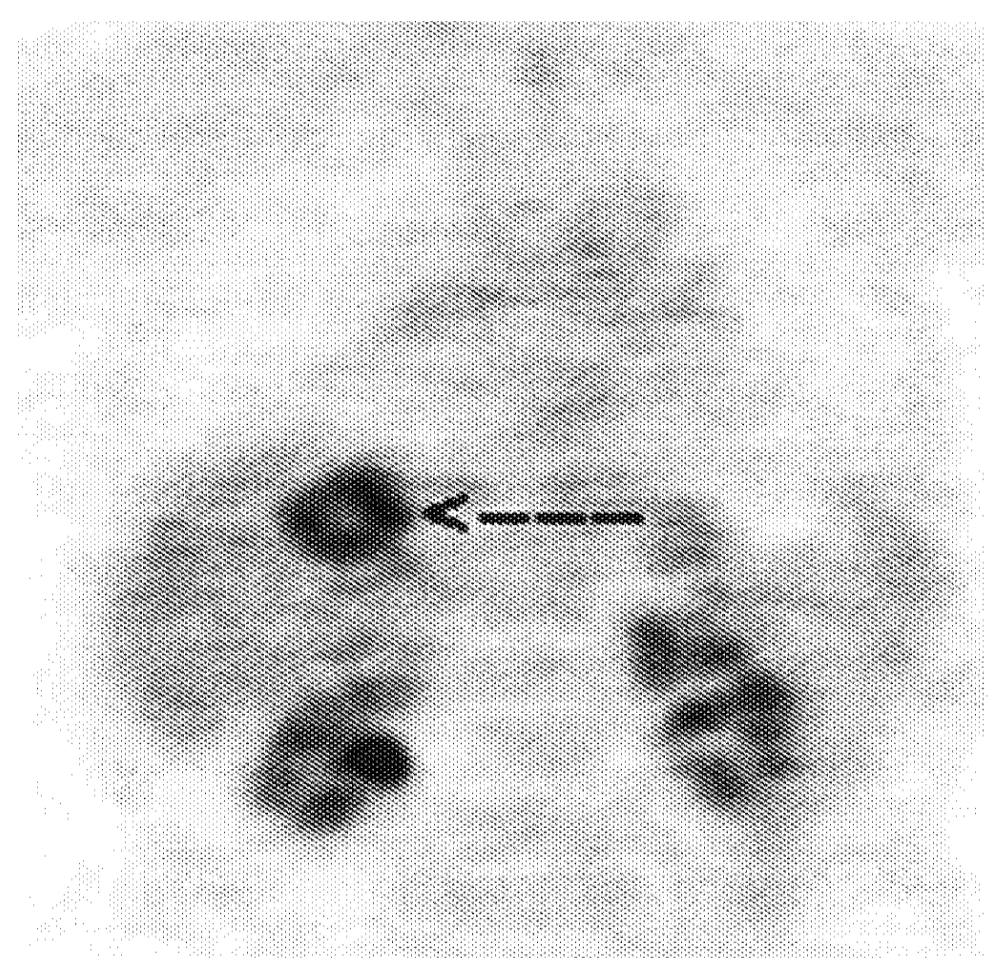

Figure 2: FDG-PET detecting liver metastasis. The woman with breast cancer has been treated with surgery and radiotherapy. During the follow-up US detected a suspicious hepatic nodule that was described as a benign hemangioma by $\mathrm{CT}$. FDG-PET showed intense uptake of FDG within the mass correctly suggesting a liver metastasis (5).

Table 2: Use of PET in Breast Cancer Patients with Distant Metastasis.(16).

\begin{tabular}{|l|c|c|c|l|}
\hline \multicolumn{1}{|c|}{ Study } & N & Sensitivity & Specificity & Author's Conclusions \\
\hline $\begin{array}{l}\text { Gallowitsch et al, } \\
(27)\end{array}$ & 62 & $97 \%$ & $82 \%$ & $\begin{array}{l}\text { In clinically suspicious cases } \\
\text { with negative tumor marker, } \\
\text { PET was reliable in detecting } \\
\text { metastasis }\end{array}$ \\
\hline $\begin{array}{l}\text { Eubank et al, } \\
(28)\end{array}$ & 61 & $94 \%$ & $91 \%$ & $\begin{array}{l}\text { PET altered the therapeutic } \\
\text { plan in 32\% of patients }\end{array}$ \\
\hline Fueger et al, (29) & 58 & $94 \%$ & $84 \%$ & $\begin{array}{l}\text { Compared PET/CT with PET } \\
\text { alone and found equivalent } \\
\text { results }\end{array}$ \\
\hline Weir et al, (30) & 27 & $89 \%$ & $88 \%$ & $\begin{array}{l}\text { Distant metastases were } \\
\text { demonstrated in 30\% of } \\
\text { patients who were thought } \\
\text { only to have loco regional } \\
\text { recurrence }\end{array}$ \\
\hline $\begin{array}{l}\text { Ladheer et al, } \\
(31)\end{array}$ & 25 & $95 \%$ & $20 \%$ & $\begin{array}{l}\text { In primary breast cancer } \\
\text { setting, patient management } \\
\text { was changed for 5 women }\end{array}$ \\
\hline
\end{tabular}




\subsubsection{Response to Therapy}

FDG-PET can measure the metabolic activity of the tumors. It is widely known that metabolic response of the tumor is prior to the physical changes in dimensions which can be measured by the standard imaging modalities like CT, MRI etc. This is because the treatment given to the patient first affects the metabolism of the cancer cells and only at a later stage the tumor size. Decrease in FDG uptake is considered as the treatment being responsive while an increase in FDG uptake is considered as tumor progression and non-response to treatment. Other radiopharmaceuticals like ${ }^{11} \mathrm{C}$-labeled methionine (MET) and ${ }^{11} \mathrm{C}$-labeled tyrosine (TYR) have been used as alternatives to FDG to evaluate the response after therapy (5)(18).

\subsection{Justification of PET}

The role of PET in breast cancer management is evolving. FDG-PET has great potential to differentiate the cancer cells from the healthy tissue. The large number of clinical studies conducted proves that PET is highly sensitive to breast cancer and FDG-PET has an excellent role to play in diagnosing breast cancer. As explained above FDG-PET contribution in axillary lymph node staging is being explored while it is superior to traditional imaging modalities in diagnosing distant metastasis. FDG-PET is also useful in monitoring the therapy response of a patient. FDG-PET has a great potential in reducing the mortality due to breast cancer and has a wide range of applications in breast cancer management. Development of the dual modality PET/optical imager to assess the margins of the excised breast tissue is an attempt to utilize the potential of FDG-PET in decreasing the rate of positive margins and minimize the wait time during the surgery. This may also lead to a decrease in the number of resections required in the follow-up surgeries which is due to the inadequate margin definition during the initial surgery. 


\subsection{Margin Assessment Methods}

\subsubsection{Current Status}

In $11-60 \%$ of the lumpectomy surgeries, a second surgery is required for complete tumor resection (32). In order to evaluate the size of the tumor, a mammogram of the breast is performed. Preoperative wire localization has been the standard procedure to guide the surgeon for tumor resection for small and nonpalpable lesions. The tumor areas are marked under the guidance of the wire. Once the wire is inserted the surgeon uses the mammogram to estimate the breast tissue to be removed and the depth of the tumor relative to the entry site of the wire. The tumor with an acceptable margin is excised from the patient's breast. After the surgery a mammogram or US is taken of the specimen to ensure complete resection or negative margins. If the margins are positive resection is done during the same time or as a separate procedure(32).

According to a survey conducted by S. L. Blair et al (33), the current surgical practices followed by the surgeons to achieve negative margins are highly varied and there is no standardized procedure. The issue of margin analysis remains controversial and is to be addressed for minimizing the rate of local recurrence and the number of re-excisions. Very few surgeons did intraoperative margin assessment while only $48 \%$ did pathological margin examination. Margin assessment methods like frozen section analysis or imprint cytology are rarely done. The definition of acceptable or negative margins differed with surgeons. Table 3 shows the results of the survey and the large variation in the definition of acceptable margins. The first column in the table gives the acceptable minimum margins while the second column gives the percentage of surgeons who accept the respective margins.

Table 3: Acceptable margins for DCIS and invasive carcinoma by surgeons(33)

\begin{tabular}{|c|c|}
\hline Negative Margins & Percentage of Surgeons \\
\hline Any negative margin & $50 \%$ \\
\hline $1-\mathrm{mm}$ & $28 \%$ \\
\hline $2-\mathrm{mm}$ & $50 \%$ \\
\hline $5-\mathrm{mm}$ & $12 \%$ \\
\hline $10-\mathrm{mm}$ & $3 \%$ \\
\hline
\end{tabular}




\subsubsection{Other Potential Approaches}

A study conducted by Mendez JE (7) reports that margin assessment methods followed by the surgeon may alter the results thereby affecting re-excisions, treatment plan and the cosmetic outcome. Many studies show that local recurrence of cancer is at a higher rate for patients with positive margins compared to the patients with negative margins. A few of the practiced margin assessment methods are discussed in this section.

DeJean P et al (32) conducted a study to analyze an intraoperative 3D ultrasound system for tumor margin determination in breast conservation therapy. They have developed a portable, intra-operative, cost-effective 3DUS system to increase the surgical accuracy by providing the tumor geometry relative to the inserted guide wire. They claim that the system has the potential to reduce the number of re-excision surgeries and reduce the operative time for the patient with anesthesia (32).

Another study done at the University of Florida by Camp ER, et al (6) analyzed the rates of re-excisions, locoregional recurrences and survival rates by conducting frozen section analysis of shaved breast tissue from the cavity bed. 1-cm of margin of healthy tissue was considered as negative margin. Frozen section analysis when compared with the permanent margin analysis led to lower re-excision rates though there was no difference in locoregional recurrence rate. Though this technique has advantages in terms of cosmetic outcome and re-excision rates it is time consuming and expensive (6).

Hughes $\mathrm{JH}$ et al (34) conducted a multi-site trial of tumor localization using a radioactive seed as an alternative to the standard wire localization procedure. For invasive and in-situ disease margins $\geq 2 \mathrm{~mm}$ were considered as negative margins. Negative margins were achieved in $73 \%$ of the radioactive seed localization (RSL) patients compared to $54 \%$ in wire localization (WL) patients. A re-excision was done in $8 \%$ of the RSL patients versus $25 \%$ of WL patients. Results obtained from this trial show that RSL has the potential to reduce the rate of positive margins and decrease the number of second surgeries to be performed (34). 
A study conducted by Paredes $\mathrm{P}$ et al (35), used a portable intraoperative gamma camera to evaluate the re-excision of non-palpable breast cancer lesions. The patients were injected with 99m Tc-labelled nanocolloid a day before the surgery. The surgery was done under the guidance of a gamma probe and the images of the surgical bed and the excised specimen were taken with the portable gamma camera. Also a $99 \mathrm{mTc}$ pointer of $11 \mathrm{MBq}$ was used to visualize the outline of the excised breast tissue. The pointer gave the flexibility to draw an appropriate outline image of the tissue with lower intensity than that of the tumor. The results obtained using the portable gamma camera, were in accordance with the pathological results by $60 \%$. Figure 3 shows the results obtained using the gamma camera (35).
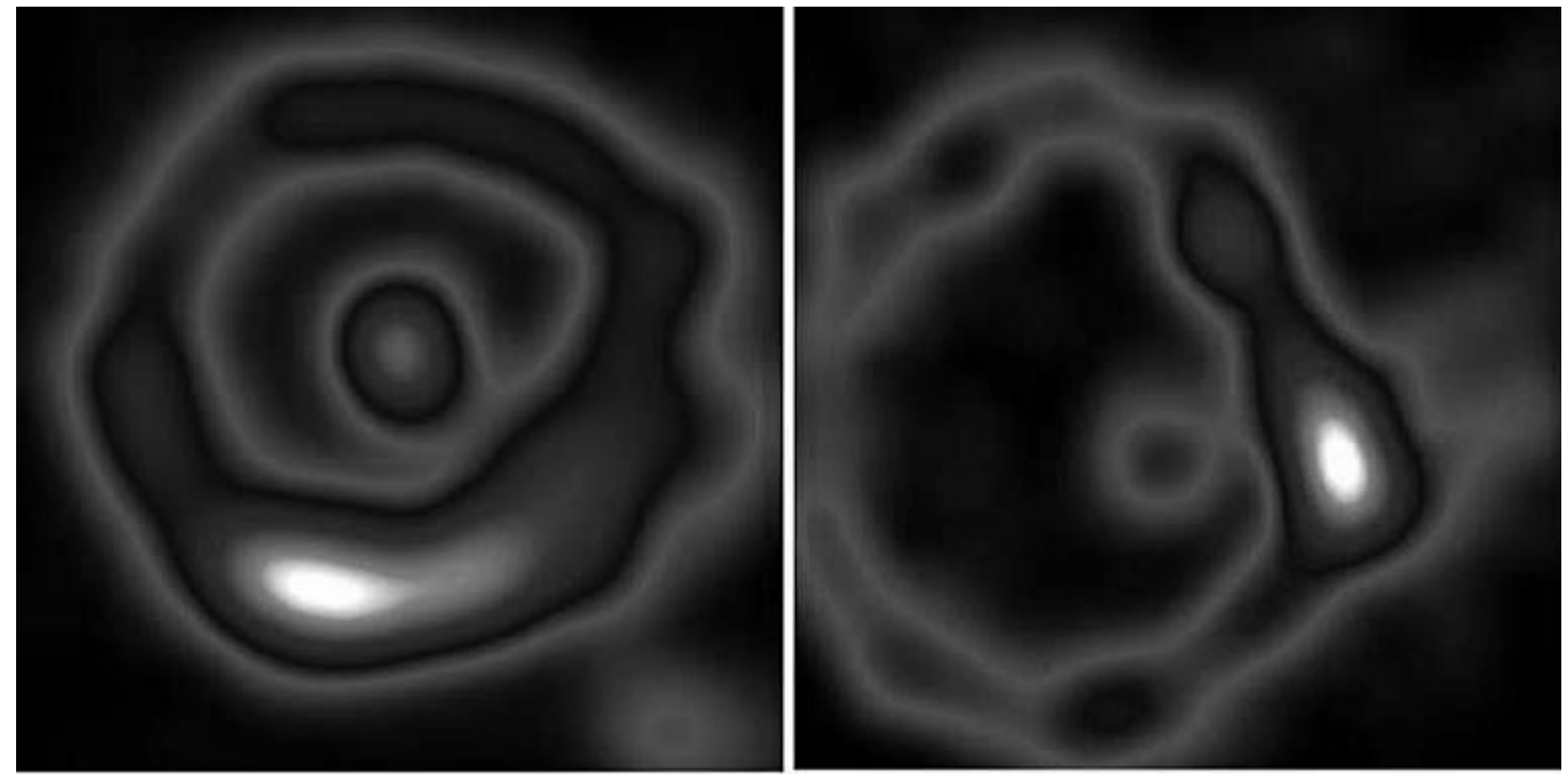

Figure 3: Images obtained with the portable gamma camera. On the left the image shows the tumor centered inside the specimen. However, on the right, the image clearly demonstrates the close proximity of the tumor to the 3 o'clock margin (35)

As discussed earlier there is no consensus on optimal techniques for margin assessment in breast conservation therapy. The rate of positive margins remains high despite using various strategies. Therefore there is a need to further investigate the margin assessment methods to reduce the number of re-excisions and the rate of positive margins. Hence the current study of development of dual modality PET/Optical imager gains importance as a potential technique to reduce the number of re-excisions and decrease wait time during surgery. 


\section{Materials and Methods}

\subsection{Overview of the solution}

Dual modality PET /Optical imager is a compact table top/mobile 3D imaging system which uses the dual modalities Positron Emission Tomography and Optical modality for imaging. PET is used to identify the cancerous lesion in the excised breast tissue while optical modality is used to provide the physical outline of the tissue. The margins are evaluated after merging the individual 3D volumes obtained from PET and optical systems. If the margins are less than 5 millimeters it is considered as positive margins are obtained which indicates further resection is required. Alternatively if the margin is greater than 5 millimeters it is considered that negative margins are obtained and the entire cancerous lesion is removed successfully from the patient. Using the Dual modality PET/Optical imager for margin analysis is an intraoperative procedure which can potentially reduce the operating time and the requirement for a second surgery.

Positron Emission Tomography with F-18 Flouro-deoxyglucose (FDG) has proved to play an important role in breast cancer imaging. The patient will be subjected to intravenous injection of FDG before the surgery. Cancer tumors show an increased uptake of FDG which allows them to be imaged in non interventional methods using PET imagers. In a few of the cases, the patient may have a wire inserted into the center of the lesion under the guidance of mammography to provide additional information about the location of the center of the lesion. Using the information from diagnostic imaging and from the wire location the surgeons remove the cancerous lesion. In order to map the specimen orientation the surgeon places one short stitch, at the superior specimen margin, which is marked as a 12 o' clock reference and 3 o' clock reference is marked with a long stitch in the same plane which is referred as lateral. Also the excised tissue and the surgical bed may be marked with dyes in order to retain the specimen orientation and for margin analysis. Currently the maximum size of the specimen can be about $3 "$.

The excised breast tissue is placed in the dual modality PET /Optical imager for margin assessment. It is placed on the specimen tray and rotated by limited angles as required by the PET and Optical 3D reconstruction. The specimen holder is placed in between two planar PET 
detectors. The PET cameras image the cancerous lesion in the excised breast tissue. The specimen is rotated from 0 to 180 degrees for PET 3D reconstruction in steps of 10 degrees. The cancerous tissue will typically be identified as a focal area or areas of increased radiotracer accumulation within the excised specimen. Slices are generated from the 3D reconstructed PET images of the cancerous lesion and exported as a binary file.

The PET/Optical imager uses a Prosilica GC-750C camera to do the 3D reconstruction of the physical surface of the excised breast tissue. Optical images of the specimen are obtained after PET acquisition. The specimen tray is made to rotate from 0 to 350 degrees in steps of 10 degrees to collect complete information about the surface of the specimen. A photograph is taken by the camera every 10 degrees. 35 images are taken as the input and 3D reconstruction of the surface is done using Matlab. Slices are generated from the reconstructed 3D point cloud.

Slices obtained from the PET system are merged with their corresponding slices obtained from optical system using a calibration procedure for proper merging. After merging, the slices consist of the physical outline of the excised tissue obtained from the optical modality and the cancerous lesion obtained from the PET modality. The margins are assessed to determine if adequate margins have been obtained or a re-excision is required. The process takes approximately 15 minutes.

\subsection{System Design}

\subsubsection{Key components of the Dual Modality PET/Optical Imager}

- One planar PET pair with typically $10 \mathrm{~cm}$ x $10 \mathrm{~cm}$ active field of view and $\sim 1.0-3.0 \mathrm{~mm}$ intrinsic spatial resolution.

- Prosilica GC 750C color camera

- Flexible LED strip

- $\quad$ Samsung CCTV, 5.5- 33 mm varifocal, manual iris lens

- Velmex rotary table, B4800TS

- Animatics smart motor SM2316D-PLS2

- Petri dish with 5" diameter

- OR-compatible mobile cabinet with shelves, and the PET/optical imager on top, for housing electronics/computers 
- Computer(s) with monitor, keyboard and mouse

- Imager signal processing electronics with low voltage and high voltage power supplies

- $\quad$ PET imager data acquisition system (hardware)

- Data acquisition and processing software

- Image reconstruction software, optionally on the second computer

- Medical quality USB/isolation transformer to assure uninterrupted power during surgery and to provide electrical safety buffer

The imager is a table top/mobile compact 3D PET/Optical imaging system. It consists of a pair of detector heads placed on fixtures to maintain constant separation between them, a rotary table and a motor to rotate the specimen, an optical camera to provide the physical outline of the specimen, a LED strip to provide back lighting for optical reconstruction and a specimen holder to hold the specimen. A holder is fixed on the back side of the box to hold the camera and half of the imager top is covered with a transparent lid for easy monitoring while the image process is on. The box is provided with handles for easy handling and portability. The mobile cabinet holds CPU, electronics control box, data acquisition system, UPS/Isolation transformer and has an arm to hold the monitor. The imager is placed on top of the OR-compatible cabinet that supports all the components of the system as shown in Figure 4. 


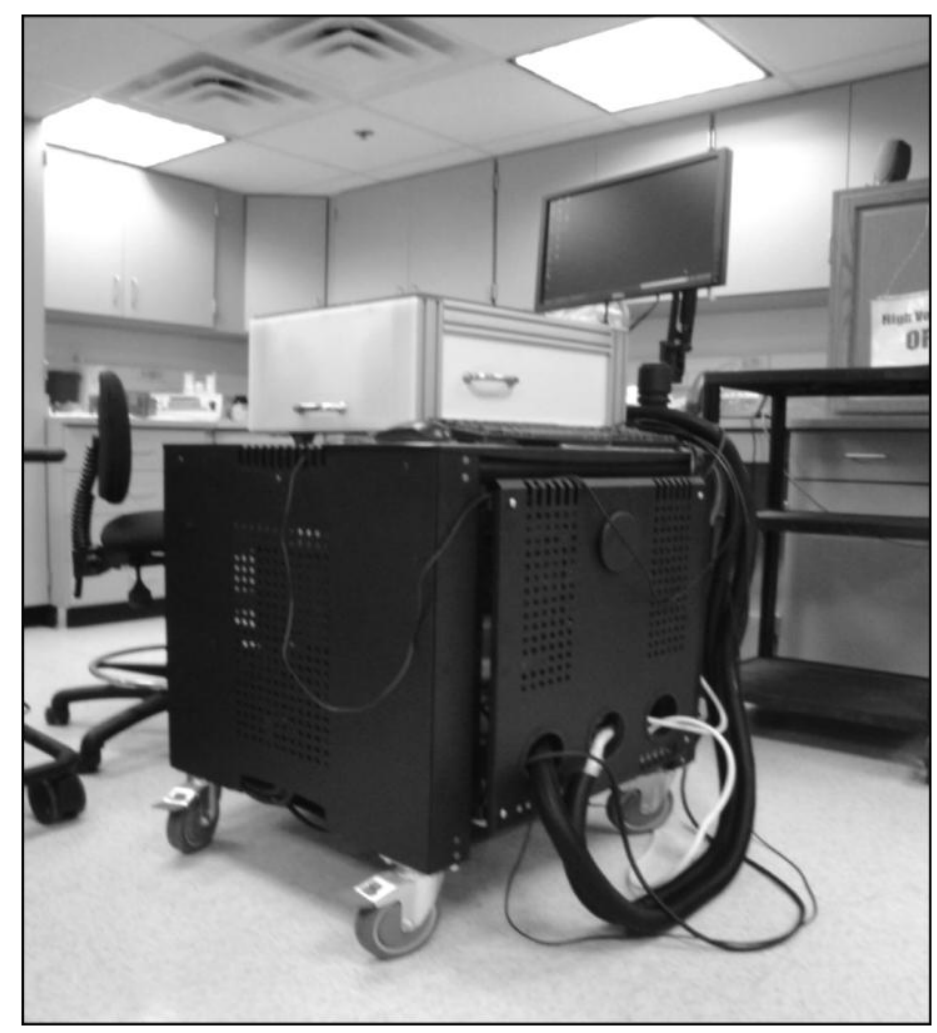

Figure 4: Dual Modality PET/Optical Imager

The positions of the camera, PET imagers, rotary table and the specimen holder are as shown in Figure 5. The camera is placed on the back of the box as explained earlier. A hole is drilled on the back wall of the imager to provide an opening for the camera view. The camera is placed such that the camera's field of view is perpendicular to the PET camera's field of view. Figure 6 shows the rotary table and the smart motor which are used to rotate the specimen holder. 


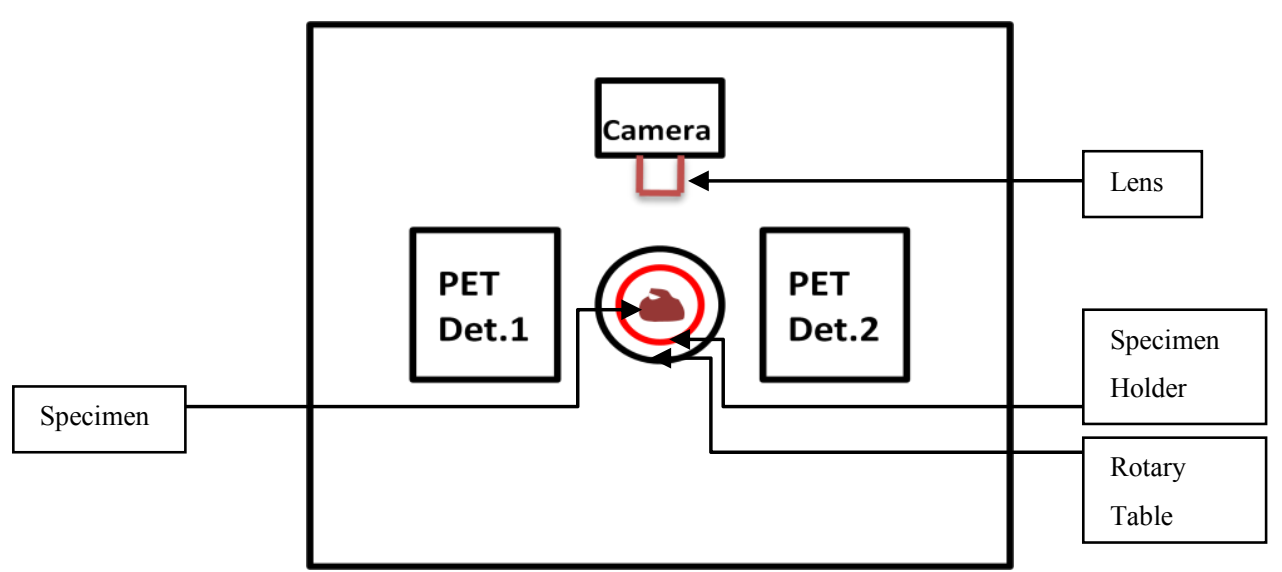

Figure 5: Schematic Diagram of the Top View of the PET/ Optical Imager

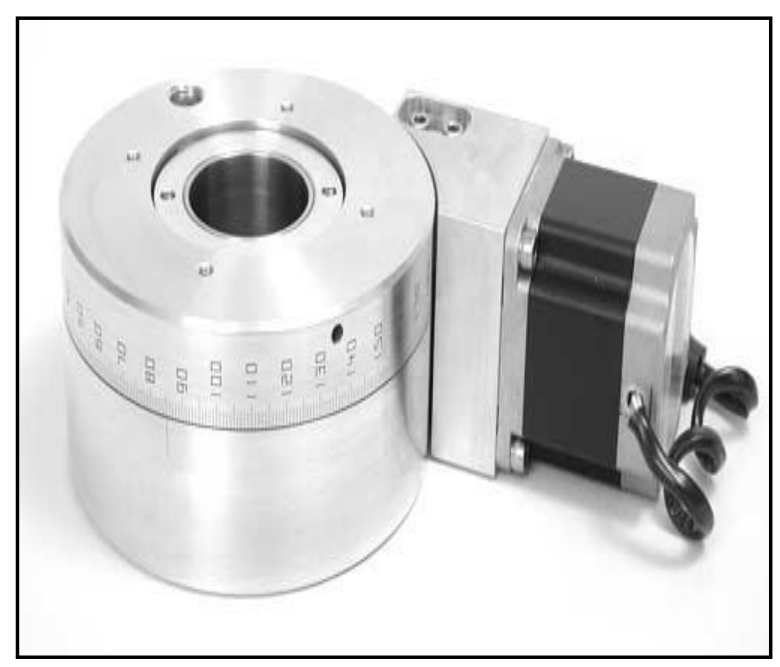

(a)

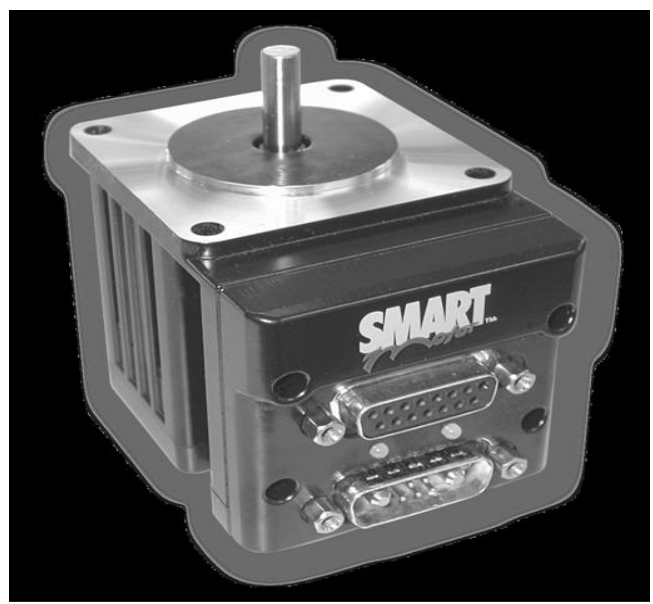

(b)

Figure 6: (a) Rotary Table (b) Smart Motor

The excised specimen is placed on a pedestal between two planar PET detectors as shown in Figure 7. The pedestal is used to provide the required elevation to the specimen to keep it within the field of view of the optical camera and the PET detectors. An LED strip is attached on to the walls of the imager to provide back lighting within the box. Figure 8 shows all the views of the imager. 


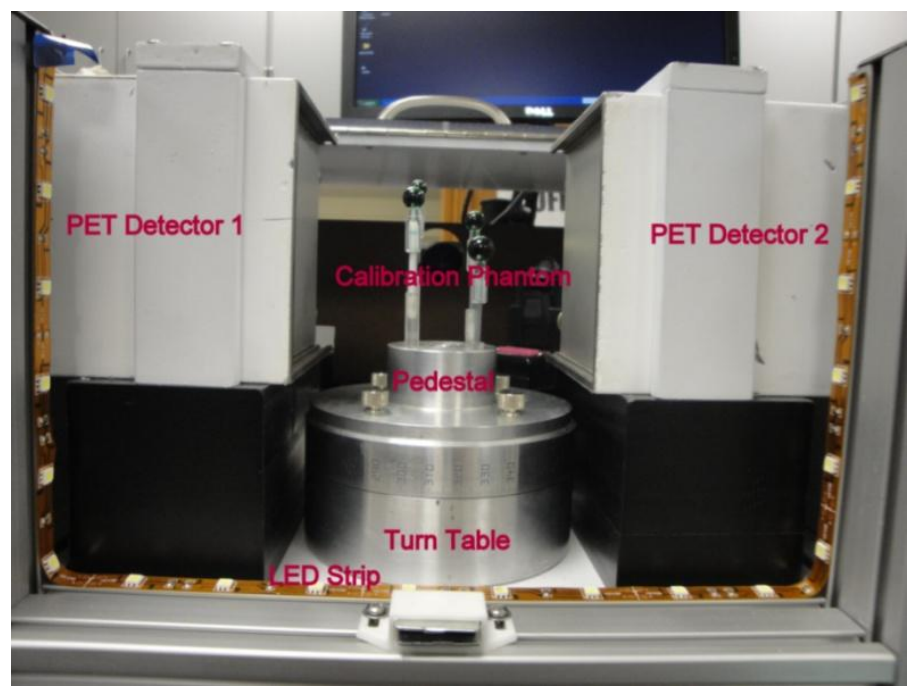

Figure 7: Components of the PET/Optical imager

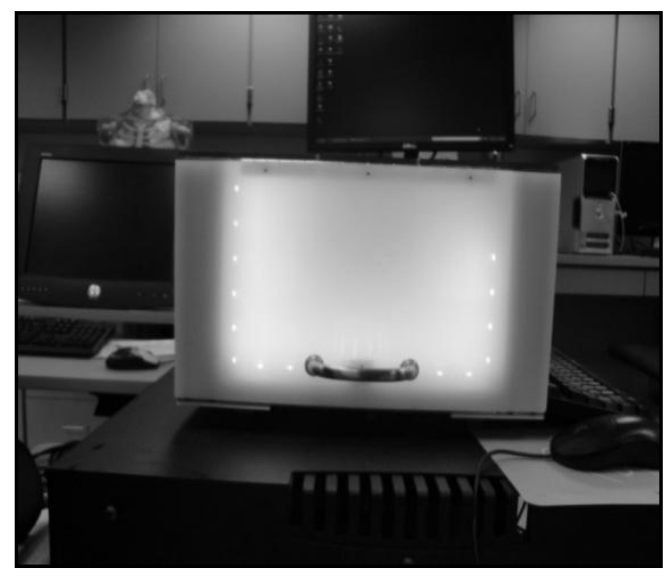

(a)

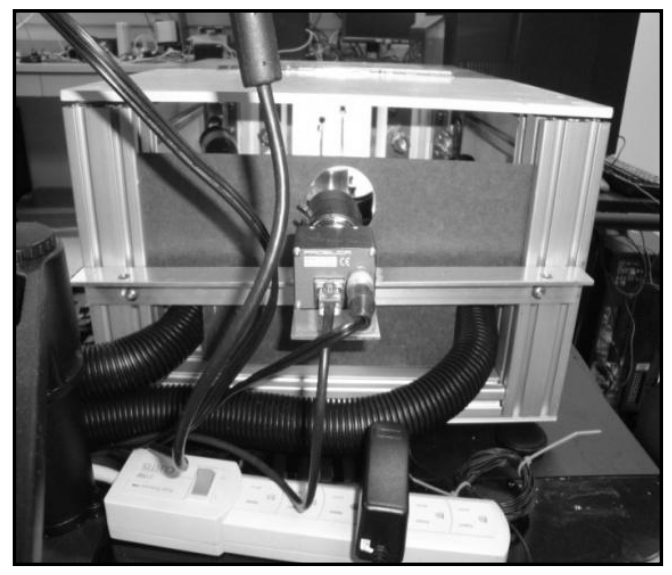

(c)

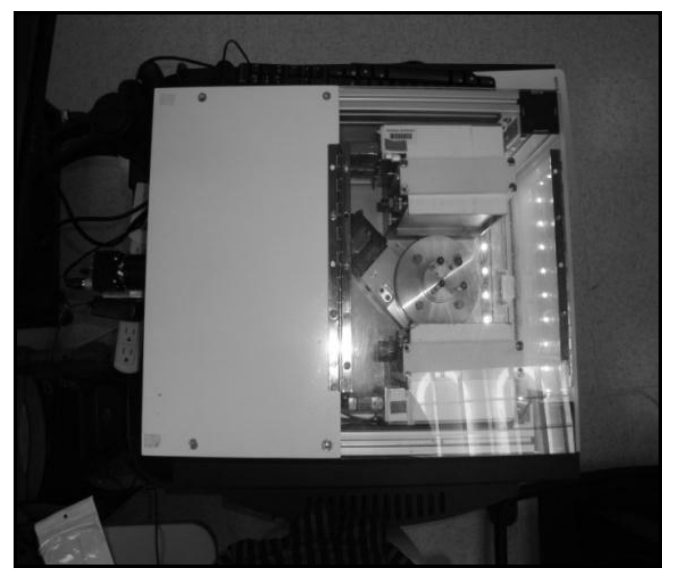

(b)

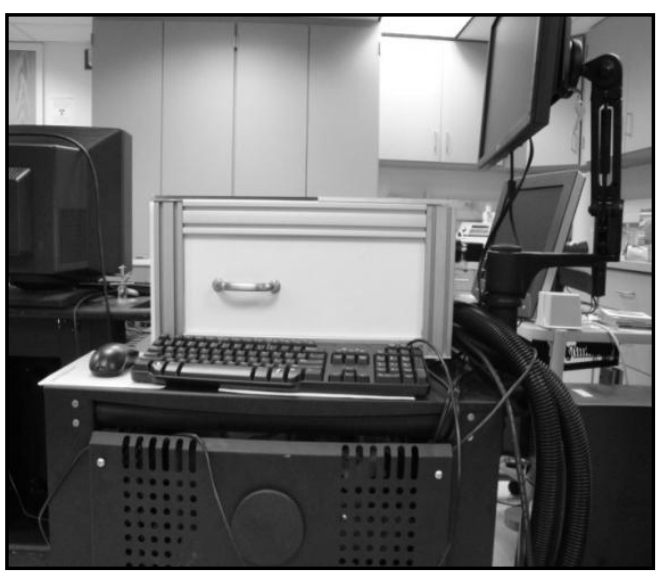

(d)

Figure 8: Images of the PET/Optical Imager. (a) Front View (b) Top View (c) Back View (d) Side View 


\subsection{PET Modality}

The excised specimen with radioactive tracer is placed in the specimen holder between the pair of detectors. The field-of-view of the detectors is approximately $10 \mathrm{~cm}$ square. Each PET detector is comprised of 4 by 4 arrays of Hamamatsu R5900-C8 position-sensitive photomultiplier tubes. PSPMTs are coupled to a pixellated array of GSO scintillating crystals with each crystal being $3 \mathrm{~mm} * 3 \mathrm{~mm} * 10 \mathrm{~mm}$. The signals from the PSPMTs are read using readout reduction schemes. A custom built 16-channel FPGA based ADC is used to read the detectors. Image acquisition software is written in Kmax (Sparrow Corporation, Port Orange, FL) which stores the data in the form of event data files. These event data files are exported to the reconstruction software. The PET system uses the program PEM_RECON to perform image reconstruction of the PET detectors by backprojection (laminography) or by the iterative maximum likelihood expectation maximization (MLEM) algorithm. Slices generated at a known distance from the reconstructed 3D image are exported as a binary file. For example slices can be taken at every 1 millimeter or so as required by the user (36).

\subsection{Optical Modality}

The Optical modality provides the anatomical map of the excised breast tissue. It uses a camera to provide the 3D physical outline of the specimen. The hardware and the software used to perform 3D reconstruction of the specimen are explained in this section.

\subsubsection{Hardware}

The hardware required for optical reconstruction includes Prosilica GC750C camera, Samsung lens, LED strip, Velmex turn table, Animatics motor and a specimen holder as explained in the previous section. The Prosilica GC750C color camera captures upto 60 frames per second in VGA with an image resolution of $752 * 480$ and square pixels of size $6.0 \mu \mathrm{m} * 6.0 \mu \mathrm{m}$. A varifocal lens of $5.5-33 \mathrm{M}$ is used along with the camera. LED lights are used to provide backlighting which is important in acquiring the silhouettes of the specimen for optical 3D reconstruction. The specimen holder is placed on the turntable which is in turn connected to the motor. The specimen is made to rotate from 0 to 350 degrees in 10 degree intervals. 


\subsubsection{Technical Approach, Optical 3D Reconstruction}

The basic idea behind the approach used for generating 3D coordinates of the specimen surface is to find the radial (r) and angular $(\theta)$ coordinates of the object coordinate system from the camera coordinate system. The angle of rotation of the turn table is considered as the angular coordinate while the radial coordinate is calculated. The Cartesian coordinates $\mathrm{X}$ and $\mathrm{Z}$ of the object are calculated from the cylindrical coordinates $r$ and $\theta$ while $\mathrm{Y}$ is calculated from the images. The coordinate systems are shown in Figure 9.

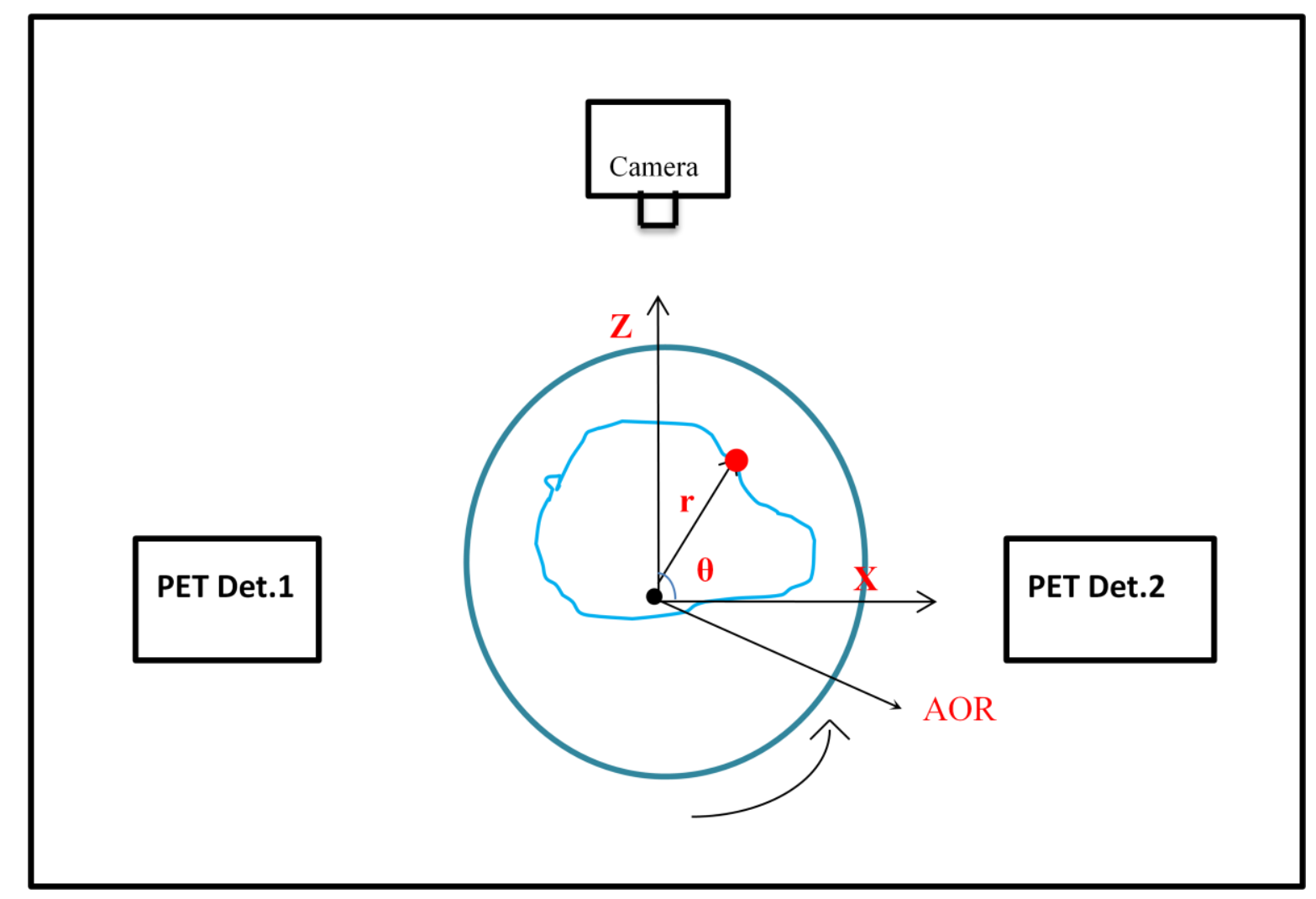

Figure 9: Top view of the DMI showing the object coordinate system

The reconstruction of the excised breast tissue is done from the multiple silhouettes. The basic principle is to generate a point cloud from the multiple view silhouettes of the specimen. The silhouette is generated by using a strip of LED lights to backlight a translucent screen behind the specimen and adjusting the camera iris manually. The Silhouette is used because the optical modality needs to describe only the physical outline of the excised tissue while the interior details of the tissue can be ignored. Multiple views of the specimen are obtained by rotating the specimen on the turn table while keeping the camera stationary as shown in Figure 10. An image 
of the specimen is captured for every 10 degrees while rotating the specimen from 0 to 350 degrees respectively.
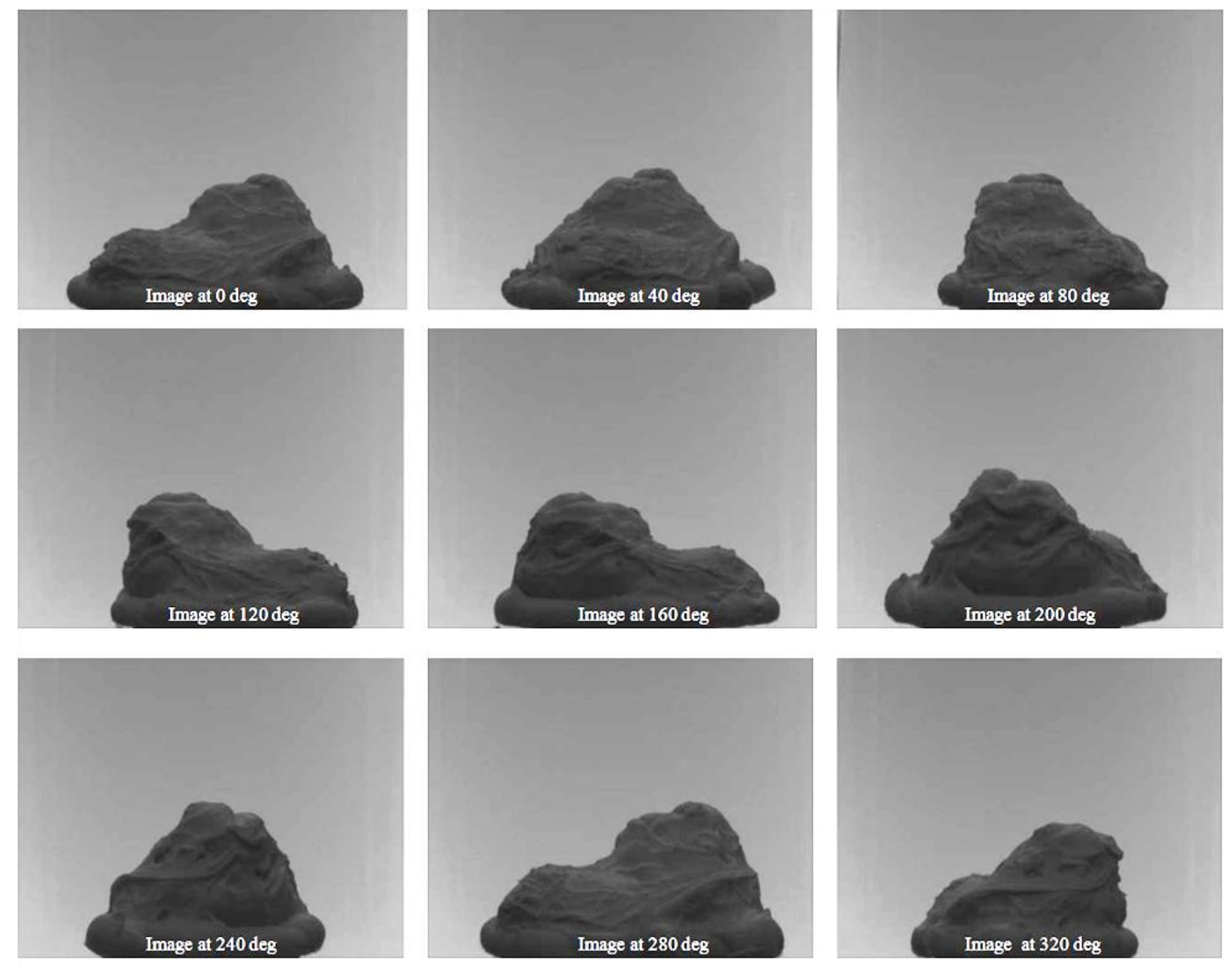

Figure 10: Multiple view silhouettes of the specimen taken at different angles

The excised breast tissue is assumed to have a convex surface for the 3D reconstruction. The specimen coordinate system is referred to as the object coordinate system and the optical coordinate system of the camera is called the camera coordinate system. Object coordinates of the physical outline are calculated from the multiple 2D camera images. The Object coordinate system is in millimeters and the camera coordinate system is in pixels. The conversion factor to convert pixels to millimeter is identified by a calibration process. $\left(\mathrm{X}_{0}, \mathrm{Y}_{0}\right)$ is the origin of the object coordinate frame. $\mathrm{X}_{0}$ and $\mathrm{Y}_{0}$ are identified in the camera coordinate frame by using a calibration process for the AOR (Axis of Rotation). The radial and angular coordinates are calculated from the 2D camera images. The Cartesian coordinates of the object are calculated 
with respect to the AOR in the object coordinate system from the cylindrical coordinates. From the multiple views a point cloud describing the surface of the tissue is obtained. The AOR is considered as $0 \mathrm{~mm}$ (millimeter) and slices are taken consecutively at required distances. The camera settings should not be changed throughout the entire process which includes the focal length, the position of the camera and the distance of the camera from the object.

The algorithm applied for 3D surface reconstruction and the slice generation is explained in the following sections.

\subsubsection{Pixel to millimeter conversion calibration}

The camera pixel to millimeter conversion factor is determined experimentally by linear transformation. A symmetrical object of known physical dimensions for example a squash ball, is taken and placed on the AOR on the turn table of the imager. Images of the object are taken for every 90 degrees from 0 to 360 degrees. The dimension of the squash ball i.e. the diameter is measured with respect to the object coordinate space using vernier calipers. The diameter of the squash ball in pixels is calculated with respect to the image coordinate system. The dimensions obtained from both the coordinate systems are used to find the pixel to millimeter conversion factor as explained in this section. The pixel to millimeter conversion factor is calculated in both horizontal (x) and vertical (y) directions and found to be the same, due to the square pixel format

of the camera. The conversion factor is a function of the geometry and the lens settings i.e. the focal length.

\subsection{Algorithm}

1. Measure the diameter of the squash ball using vernier calipers. Let the diameter be $\mathrm{D} \mathrm{mm}$ and it is the same in both horizontal and vertical directions.

2. Consider the image at 0 degrees and find the diameter of the squash ball as $d_{\mathrm{h} 1}$ pixels in the horizontal direction and $\mathrm{d}_{\mathrm{v} 1}$ pixels in the vertical direction.

3. Repeat step 1 for the remaining three images and find the diameter in the horizontal direction as $d_{h 2}, d_{h 3}$, $d_{h 4}$ pixels and the diameter in the vertical direction as $d_{v 2}, d_{v 3}, d_{v 4}$ in pixels respectively.

4. Calculate the mean diameter in horizontal direction as $d_{m h}$ pixels of $d_{h 1}, d_{h 2}, d_{h 3}, d_{h 4}$ and mean diameter in vertical direction $d_{m v}$ pixels of $d_{v 1}, d_{v 2}, d_{v 3}, d_{v 4}$

5. The pixel to millimeter conversion factor in horizontal direction then becomes 


$$
\begin{gathered}
D(m m)=d_{m h}(\text { pixels }) \\
\Rightarrow \quad 1(m m)=\frac{d_{m h}}{D}(\text { pixels }) \\
\text { Pixel2mm_Factor (horizontal) }=\frac{d_{m h}}{D}(\text { pixels for } 1 \mathrm{~mm})
\end{gathered}
$$

6. Pixel to millimeter conversion factor in vertical direction

$$
\begin{gathered}
D(m m)=d_{m v}(\text { pixels }) \\
\Rightarrow \quad 1(m m)=\frac{d_{m v}}{D}(\text { pixels }) \\
\text { Pixel2mm_Factor (vertical })=\frac{d_{m v}}{D}(\text { pixels for } 1 \mathrm{~mm})
\end{gathered}
$$

\subsubsection{Axis of rotation Calibration}

The origin of the object coordinate frame $\left(\mathrm{X}_{0}, \mathrm{Y}_{0}\right)$ is identified in the camera coordinate frame. $\mathrm{X}_{0}$ is the coordinate of the axis of rotation and $\mathrm{Y}_{0}$ is the coordinate of the top of the pedestal on which the object rests. A calibration procedure is followed to identify $\left(\mathrm{X}_{0}, \mathrm{Y}_{0}\right)$ in the camera coordinate system as explained here. A capillary tube filled with food color is placed on the AOR of the turn table as shown in Figure 11. The capillary tube is made to rotate from 0 to 358 degrees with intervals of 2 degrees. An image is taken for every 2 degrees to minimize the error.

\subsection{Algorithm}

1. Consider the first image and identify the top of the pedestal as $Y_{0}$

2. Change the color image into grayscale and identify the edges of the capillary tube in the image

3. Save the $X$ coordinates of the edges in a matrix as $X_{1} \ldots X_{n}$.

4. Calculate the mean of the $X$ coordinates of the edges in the image as $X_{M 1}$

5. Repeat the steps 2 to 4 for all the images and save the means of $\mathrm{X}$ coordinates as $\mathrm{X}_{\mathrm{M} 1}, \mathrm{X}_{\mathrm{M} 2} \ldots \mathrm{X}_{\mathrm{M} 178}$ 
6. $\mathrm{X}_{0}$ is the mean of $\mathrm{X}_{\mathrm{M} 1}, \mathrm{X}_{\mathrm{M} 2} \ldots \mathrm{X}_{\mathrm{M} 178}$

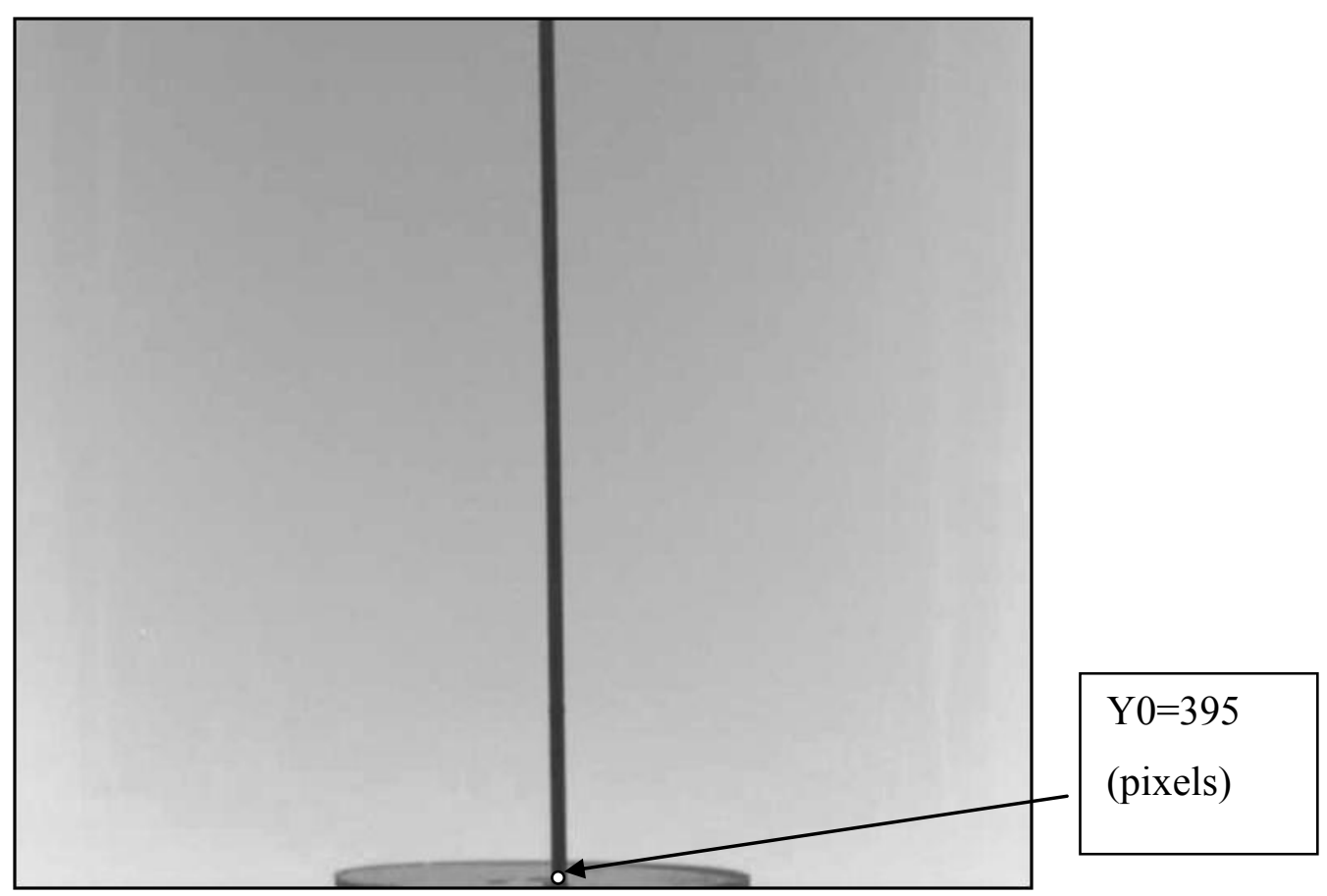

Figure 11: Capillary tube with food color

\subsubsection{Optical 3D Reconstruction and Slice Generation}

The excised specimen to be reconstructed is assumed to be convex. The camera position and the focal length settings should the same as during the calibration procedures. The specimen to be reconstructed is placed on the AOR of the turn table and rotated from 0 to 350 degrees and an image is taken for every 10 degrees. The Pixel to millimeter conversion factor is obtained from the calibration procedure and the AOR is identified in the image coordinate frame from the AOR calibration as $\left(\mathrm{X}_{0}, \mathrm{Y}_{0}\right)$. The height at which slices are to be generated from the reconstructed phantom is specified which can typically be $0.5 \mathrm{~mm}$ or $1 \mathrm{~mm}$ etc. as specified by the end user. The camera coordinate system and the object coordinate systems are as shown in Figure 12 and Figure 13. 


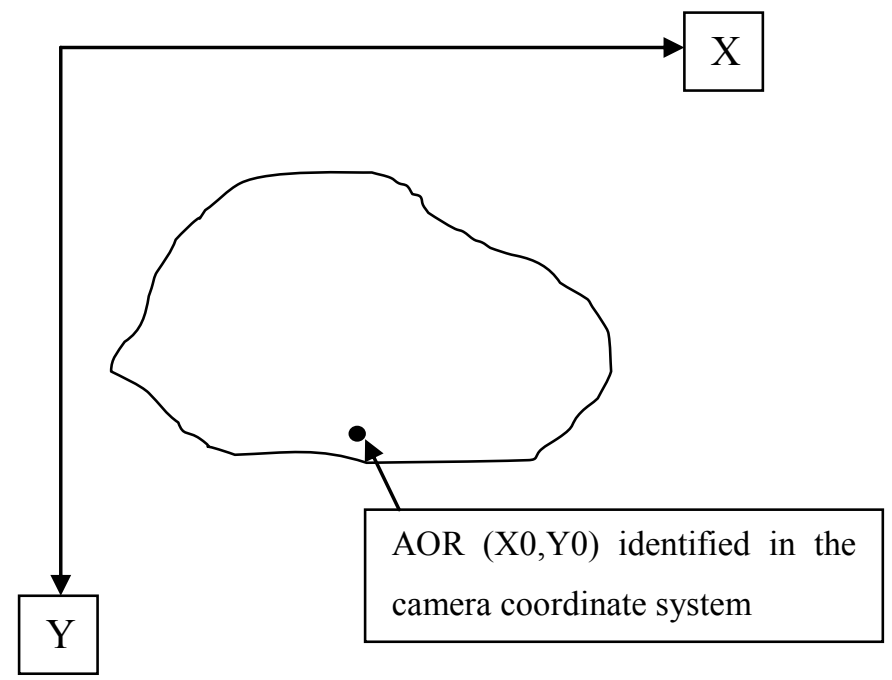

Figure 12: Image coordinate system

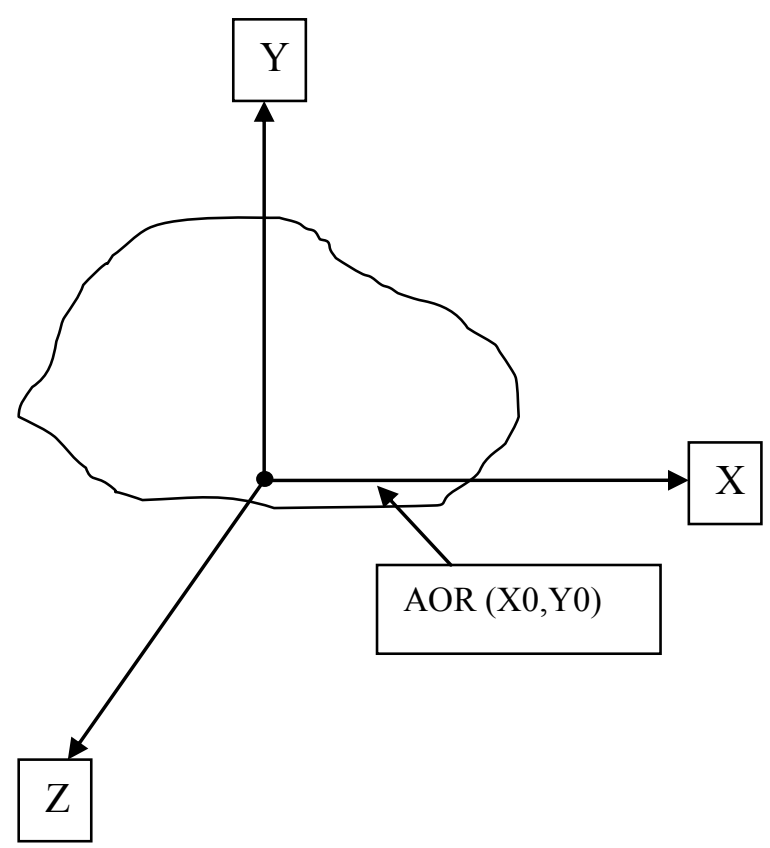

Figure 13: Object coordinate system 


\subsection{Algorithm}

1. Initialize

- conversion factor as Pixel2mm_Factor from the pixel to millimeter calibration procedure

- $\mathrm{AOR}$ as $\left(\mathrm{X}_{0}, \mathrm{Y}_{0)}\right.$ from the AOR calibration

- Degree of Rotation of the turn table as DOR=10 degrees

- Angle $=0$ to 350 degrees in steps of DOR

- Sliceheight $=1 \mathrm{~mm}$

- $\quad$ Threshold $=0.5$

- Number of images as nimg=35 which starts from 0

- Set the xlimit and y limit to crop the image

$$
\left[r_{1}, c_{1}\right]=\text { size }(\text { cropped image })
$$

2. FOR $i=1$ to length(Angle)

$$
\begin{aligned}
& C=\cos (\operatorname{Angle}(i)) \\
& S=\sin (\operatorname{Angle}(i))
\end{aligned}
$$

\section{END FOR}

3. FOR $n n=0$ to nimg

- Read Image. A sample of an image taken by the camera at 0 degrees is shown in Figure 14.
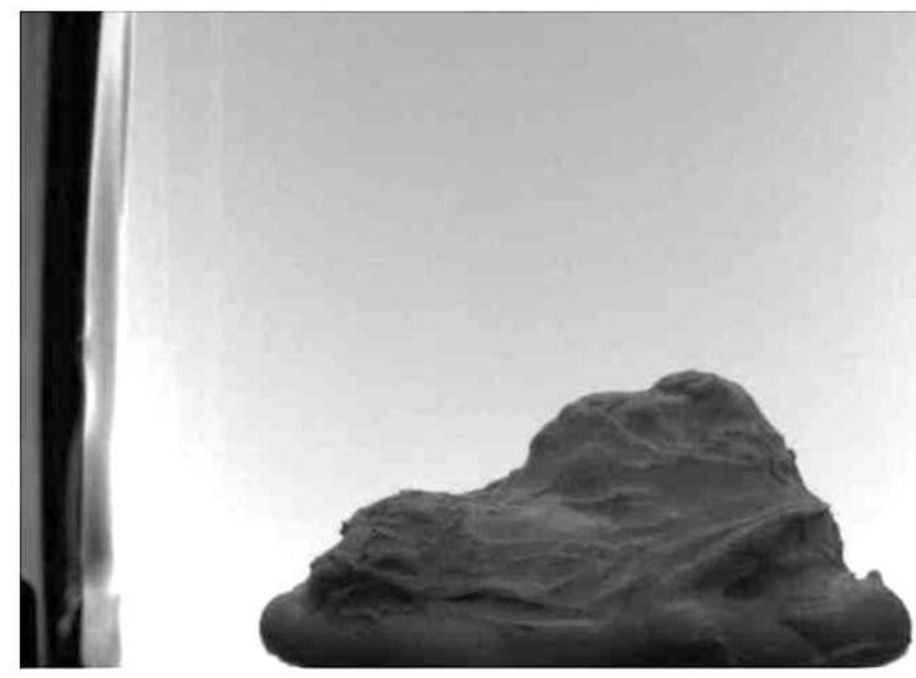

Figure 14: Camera image at 0 degrees 
- Crop the image. An example of a cropped image at 0 degrees is shown in Figure 15.

$$
\left.\left[r_{1}, c_{1}\right]=\text { size(cropped image }\right)
$$

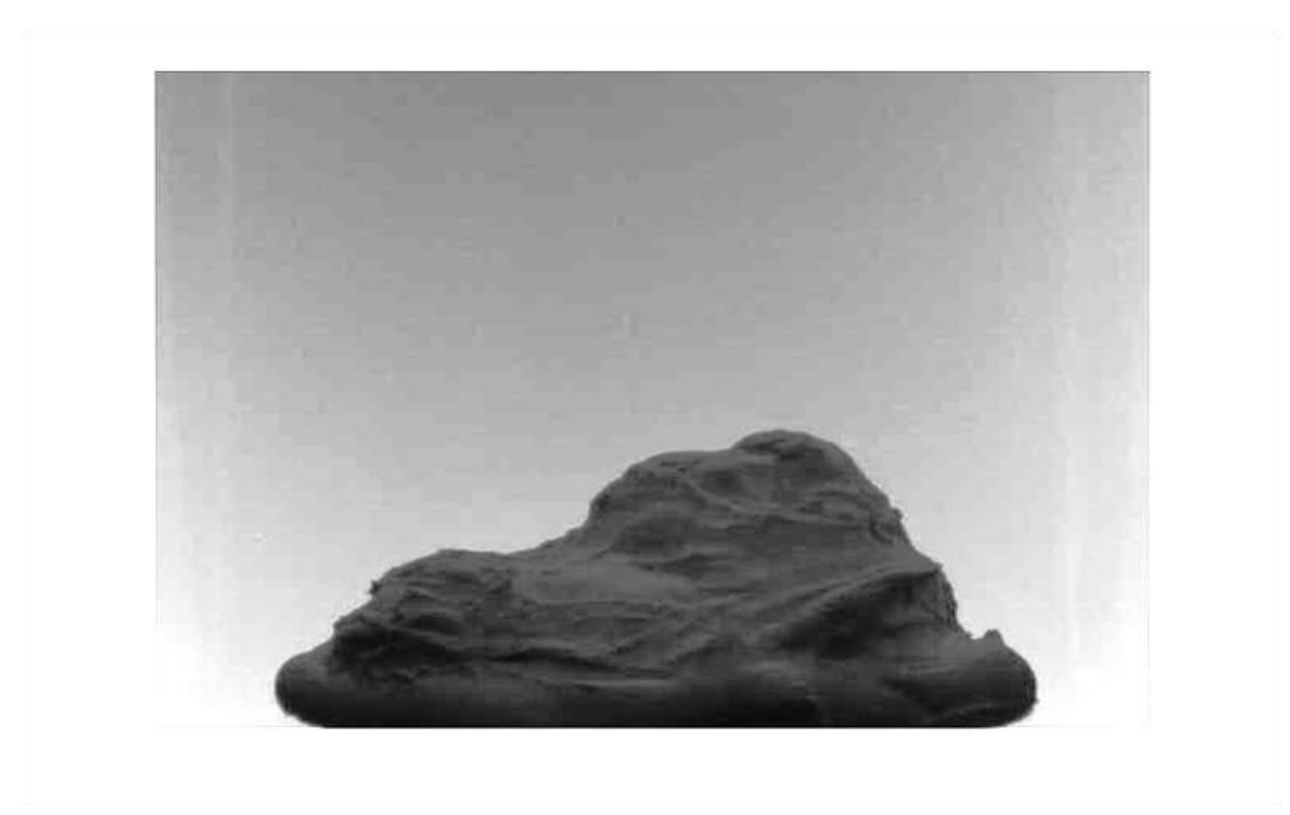

Figure 15: Cropped Image at 0 degrees

- Change the color image to grayscale image

- Detect the edges in the image using the canny edge detection technique with the specified threshold value. Figure 16 shows different images of the specimens taken at 0 degrees, 90 degrees, 180 degrees and 270 degrees with the edges identified. 

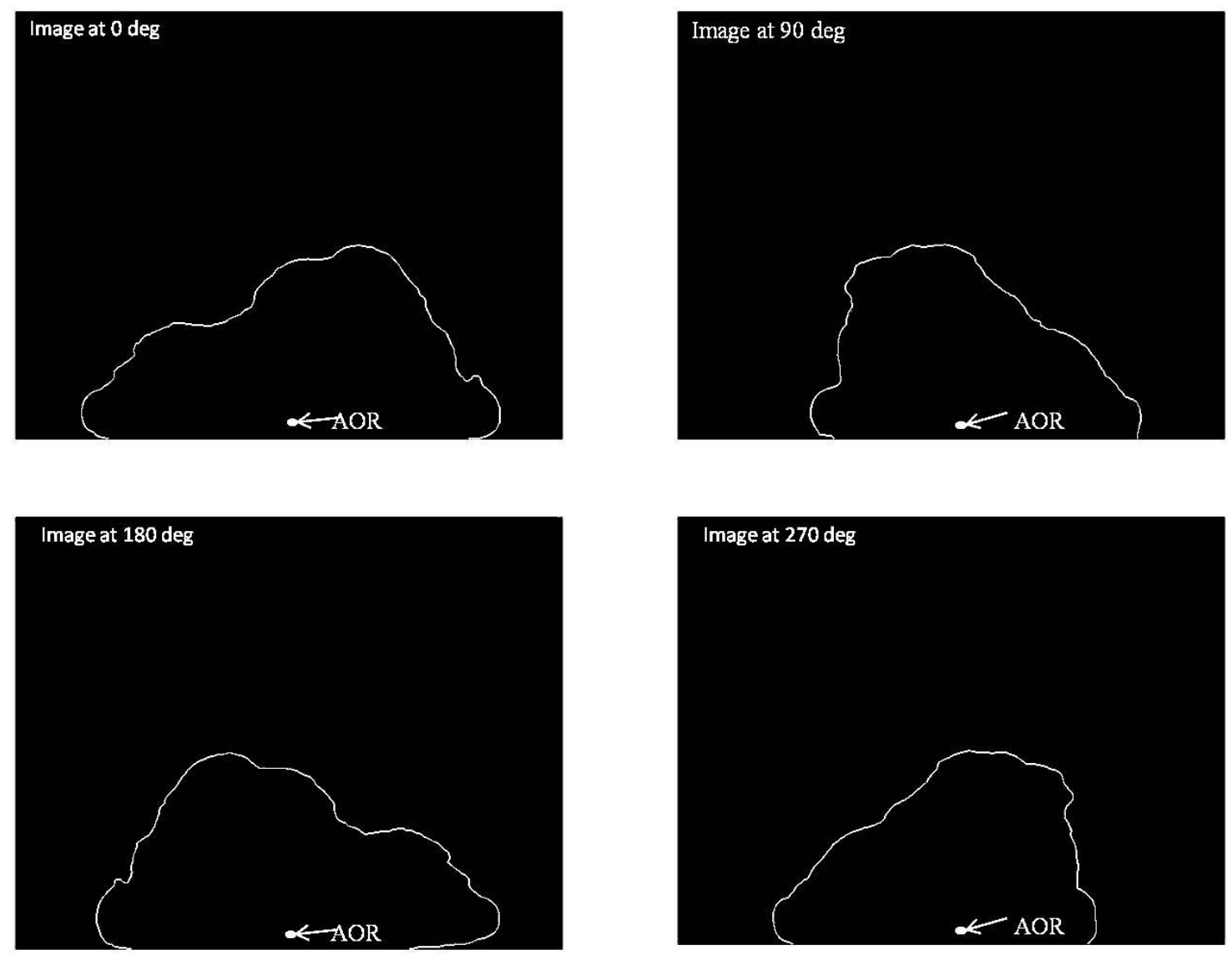

Figure 16: Edges identified for images taken at different angles

- Considering the AOR $\left(\mathrm{X}_{0}, \mathrm{Y}_{0}\right)$ in the image plane as $0 \mathrm{~mm}$ find the consecutive $\mathrm{y}$ pixel coordinates at $1 \mathrm{~mm}, 2 \mathrm{~mm}$ etc using the pixel to $\mathrm{mm}$ conversion factor

$$
y=\left(Y_{0}-\left(h * \text { Pixel } 2 m m_{-} \text {factor }\right)\right)
$$

- Starting from $0 \mathrm{~mm}$ find the $\mathrm{x}$ and $\mathrm{y}$ pixel coordinates on the left and right edges of the AOR

- At the edges compute $r$ in millimeter as follows

$$
r=\frac{\left(x-X_{0}\right)}{\text { Pixel2mm_Factor }}
$$


- At each point compute $\mathrm{X}, \mathrm{Y}$ and $\mathrm{Z}$ which are in calculated in millimeter

$$
\begin{gathered}
X=r^{*} C(n n+1) \\
Z=r^{*} S(n n+1) \\
Y=h
\end{gathered}
$$

- Store coordinates as $(\mathrm{X}, \mathrm{Y}, \mathrm{Z})$ for each image

\section{END FOR}

4. A point cloud of the surface coordinates of the object to be reconstructed is obtained where the coordinates are in $\mathrm{mm}$. The front view of the point cloud is shown in Figure 17 and the top view of the point cloud is shown in Figure 18.

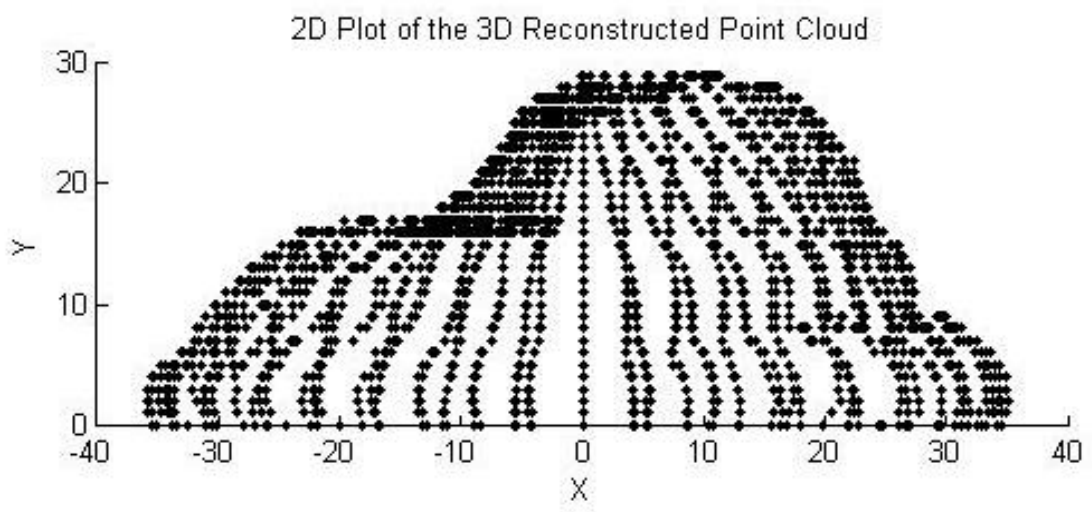

Figure 17: Front view of the point cloud 


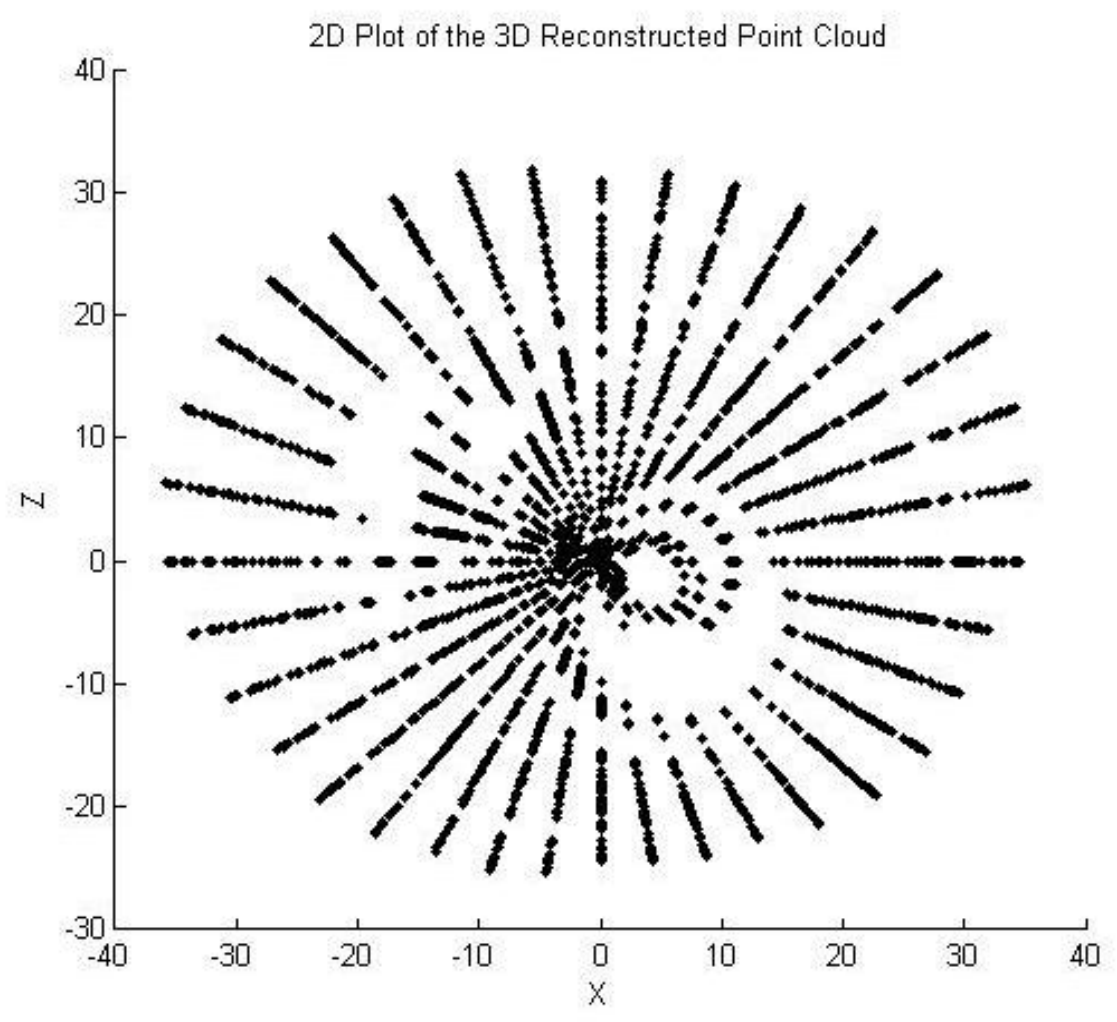

Figure 18: Top view of the point cloud

5. Calculate the arc tangent of the $\mathrm{Z}$ and $\mathrm{X}$ coordinates as below

$$
\text { Theta }=\tan ^{-1} \frac{Z}{X}
$$

6. Radius of the points on the surface is calculated from the $(0,0)$ as below

$$
\text { Radius }=\sqrt{X^{2}+Z^{2}}
$$

7. Slices are generated from minimum value of $\mathrm{Y}$ coordinates to maximum value of $\mathrm{Y}$ coordinates respectively.

8. FOR Temp_Var $=$ minimum $(\mathrm{Y})$ to maximum $(\mathrm{Y})$

- Find all the points whose $\mathrm{Y}=$ Temp_Var and save $\mathrm{X}, \mathrm{Y}, \mathrm{Z}$, Theta, R of the respective points

- Theta values range from 0 to 350 degrees. Each theta might have more than one corresponding point. From the set of points available for each theta value select 
the point with the minimum radius and save as $\mathrm{P}$. The point cloud corresponding to a particular slice is as shown below. Figure 19 shows the point cloud of the slice at $0 \mathrm{~mm}$.

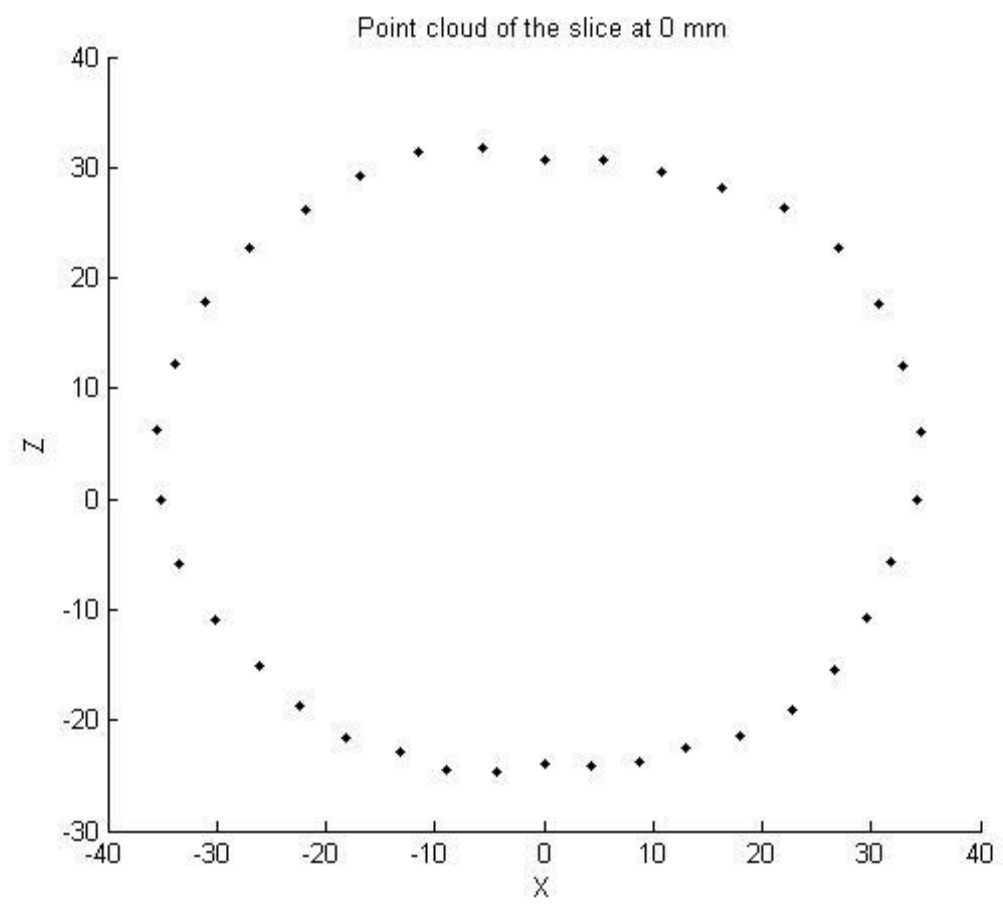

Figure 19: Point cloud of the slice at $0 \mathrm{~mm}$

- Countvar $1=1$

FOR Points $\mathrm{P}=1$ to length $(\mathrm{P})$

* Take two consecutive points $\mathrm{P}_{1}\left(\mathrm{X}_{1}, \mathrm{Z}_{1}\right)$ and $\mathrm{P}_{2}\left(\mathrm{X}_{2}, \mathrm{Z}_{2}\right)$ and calculate the difference between their $\mathrm{X}$ and $\mathrm{Z}$ coordinates

$$
\begin{aligned}
& X_{-} \text {diff }=\max \operatorname{imum}\left(X_{1}, X_{2}\right)-\min \operatorname{imum}\left(X_{1}-X_{2}\right) \\
& Z_{-} \text {diff }=\max \operatorname{imum}\left(Z_{1}, Z_{2}\right)-\min \operatorname{imum}\left(Z_{1}-Z_{2}\right)
\end{aligned}
$$

\section{IF ( X_diff $>Z$ _diff)}

$>$ Fit a polynomial $\mathrm{p}(\mathrm{x})$ of degree 1 to the points and calculate the coefficients. 
$>$ Values of ' $\mathrm{Z}$ 'are evaluated at different values of $\mathrm{X}$ and saved in the matrices as Points_X and Points_Z

ELSE

Fit a polynomial $\mathrm{p}(\mathrm{z})$ of degree 1 to the points and calculate the coefficients.

$>$ Values of ' $\mathrm{X}$ ' are evaluated at different values of $\mathrm{Z}$ and saved in the matrices as Points_X and Points_Z

\section{END}

Curve_Coords $($ countvar 1,1$)=$ Points_X

Curve_Coords $($ countvar 1,2$)=$ Points_Z

\section{END FOR}

- At the end of the above for loop a continuous curve is obtained joining all the individual points and the $\mathrm{X}$ and $\mathrm{Z}$ coordinates of all the points are saved in the matrix Curve_Coords. Figure 20 shows the slice at $0 \mathrm{~mm}$ after the curve fitting is done as explained in the above steps. 


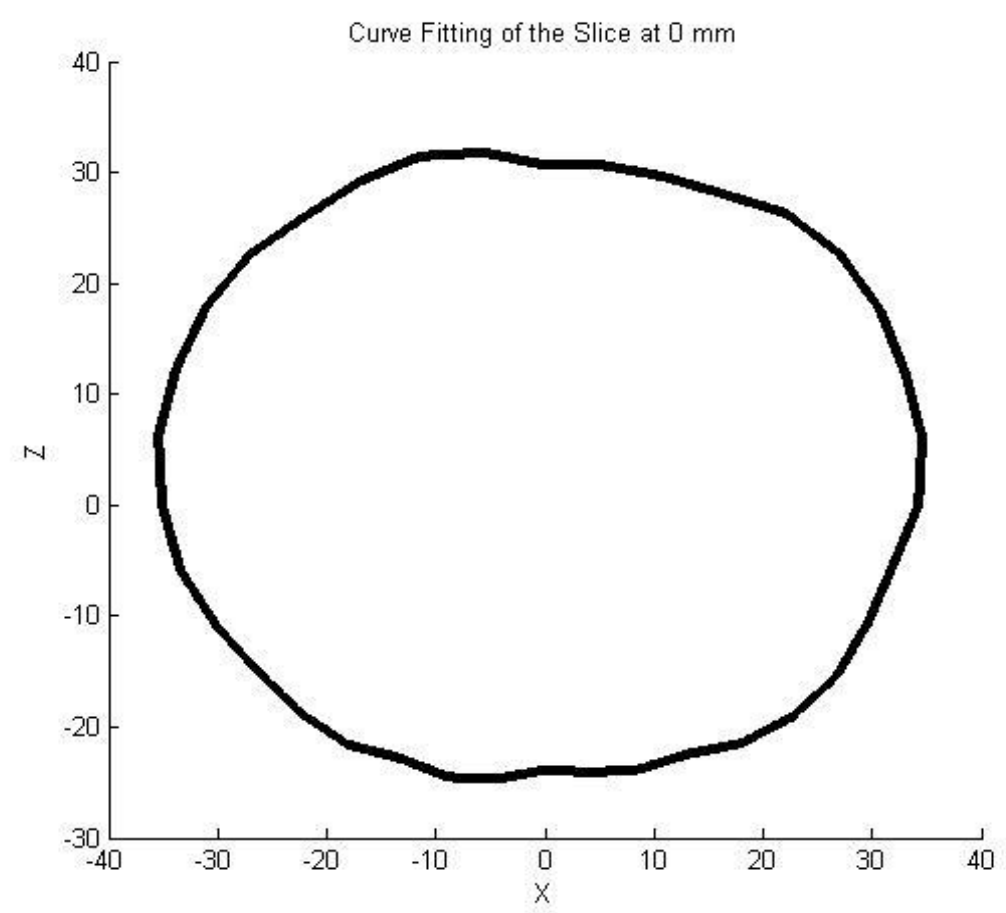

Figure 20: Slice at $0 \mathrm{~mm}$ after the curve fitting

- Change the $\mathrm{X}$ and $\mathrm{Z}$ coordinates saved in Curve_Coords from millimeters to pixels and translate the $\mathrm{X}$ coordinates by $\mathrm{Xo}$ and $\mathrm{Z}$ coordinates by $\left(\mathrm{r}_{1} / 2\right)$

$$
X Z=\text { Curve_Coords } * \text { Pixel2mm_Factor }
$$

- Maximum Y is identified as the Last_Slice

$$
\text { Last_Slice }=\operatorname{Maximum}(Y) * \text { Pixel } 2 m m_{-} \text {Factor }
$$

- Create an image, IMG1 of size $\left(\mathrm{r}_{1}, \mathrm{c}_{1}\right)$ with pixel values as zeros. Fill the pixels with the $\mathrm{XZ}$ coordinates in the image with values of 1 such that the image ultimately is a slice with the outline of the phantom placed in the imager. Figure 21 shows the image of the slice at $0 \mathrm{~mm}$ where the $\mathrm{X}$ and $\mathrm{Z}$ coordinates are in pixels. $\mathrm{X}$ and $\mathrm{Z}$ coordinates are converted from millimeter to pixels as the image merging and the display processes use the image file format. 


$$
\begin{aligned}
& I M G 1=z \operatorname{eros}\left(r_{1}, c_{1}\right) \\
& \text { FOR } j=1 \text { to length }(X Z) \\
& \operatorname{IMG} 1(X Z(j, 2), X Z(j, 1))=1
\end{aligned}
$$

END FOR

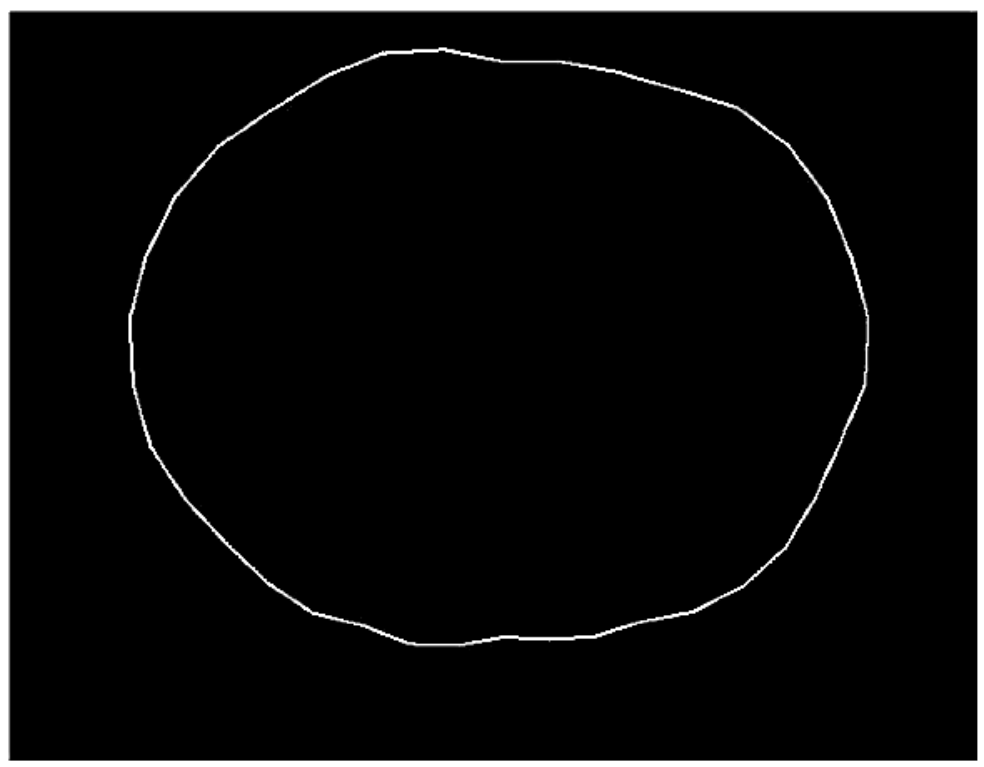

Figure 21: Image of the slice at $0 \mathrm{~mm}$ after the curve fitting

\section{END FOR}

9. The optical slices are arranged from bottom to top

10. Slices are generated from the $y=Y o$ to $y=1$ based on the slice height specified

\subsection{Image Fusion}

The slices obtained from the PET and optical modalities are merged. In order to merge these images properly a calibration procedure is followed. The calibration phantom is made of 4 glass spheres filled with dark colored radioactive solution such that it is visible to both the PET and optical modalities. The phantom is imaged with both the modalities. The centroids of the four spheres are identified in both the optical and PET modalities. Coordinates of the centroids are used to calculate the scaling, translational and rotational 
offsets between the two modalities. At the end of the calibration procedure a homogeneous transformation matrix is obtained which is recorded for further use.

A phantom made of latex caulk was constructed and holes were drilled into the phantom. Radioactive beads of $5 \mathrm{~mm}$ diameter were inserted into these holes. PET reconstruction was done and the slices were saved in a data file. The PET slices were transformed by the homogeneous transformation matrix obtained from the calibration procedure. Optical reconstruction was done in a similar way as described in the previous section. After the transformation of the PET slices, their coordinates were obtained with respect to the optical coordinate system. As both the physical outline and the radioactive beads were in the same coordinate system, the merged images were generated as explained in the following sections.

\subsubsection{Calibration}

The calibration phantom consists of four glass spheres held on glass rods as shown in Figure 22. These spheres are filled with the radionuclide FDG mixed with a dark food color. The radioactive nature of the liquid makes it visible to the PET cameras and the dark color helps in easy identification for the optical modality. The four spheres which are of different sizes and heights are placed on the turn table. Different heights are chosen for each sphere for easy identification of the spheres in both the modalities.

The coordinates of the centroids of the spheres are calculated to be $\left(\mathrm{X}_{\mathrm{op}}, \mathrm{Y}_{\mathrm{op}}, \mathrm{Z}_{\mathrm{op}}\right)$ in the optical system and $\left(\mathrm{X}_{\mathrm{p}}, \mathrm{Y}_{\mathrm{p}}, \mathrm{Z}_{\mathrm{p}}\right)$ in the PET system. Sixteen unknowns are present while finding the homogeneous transformation matrix. In order to find sixteen unknowns it is necessary to have multiple images of the four spheres. 


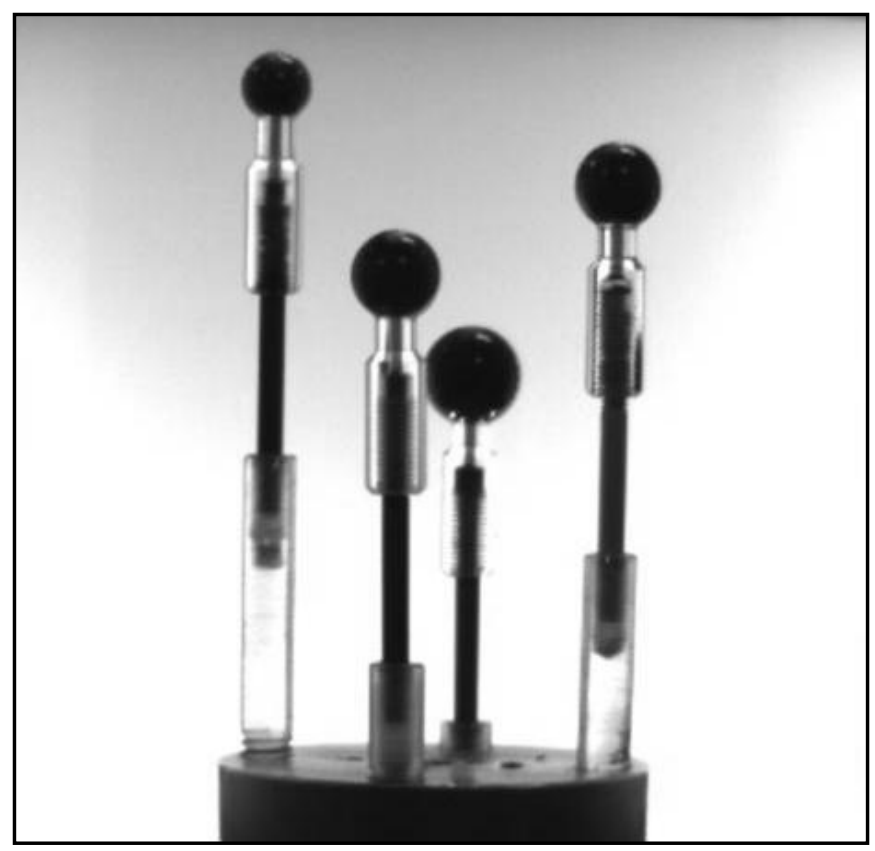

Figure 22: Calibration Phantom for Image Fusion

\subsubsection{Identification of the centroids in optical modality}

In the calibration phantom the spheres do not lie on the AOR so the degree of rotation is not known, but the difference in degree of rotation between successive images is known. An image of the four spheres where all of them are clearly visible is considered as a reference image and the angle is referred as 'Theta'. Two more images are considered whose degree of rotation from the reference image is known and referred as 'Theta1' and 'Theta2' respectively. Spheres are numbered 1 to 4 in descending order of their heights.

In order to minimize the error three sets of images are taken and the coordinates of the centroids of the spheres are an average obtained from the three sets. At least three images are required in one set to find the centroids of the spheres. The process of finding the coordinates of the centroids for one set of images is explained below.

\subsection{Algorithm}

1. Initialize

- AOR as $\left(X_{0}, Y_{0}\right)$ from the AOR calibration

- Difference in degree of rotation of the first image from the reference image as Thetal ' $\theta_{1}$ ' 
- Difference in degree of rotation of the second image from the reference image as Theta2 ' $\theta_{2}$ '

2. Read the reference image

3. Crop the reference image

$$
\left[r_{1}, c_{1}\right]=\text { size }(\text { cropped image })
$$

4. Identify the edges of the spheres alone in the image. Figure 23 shows the images of the spheres with their edges identified.

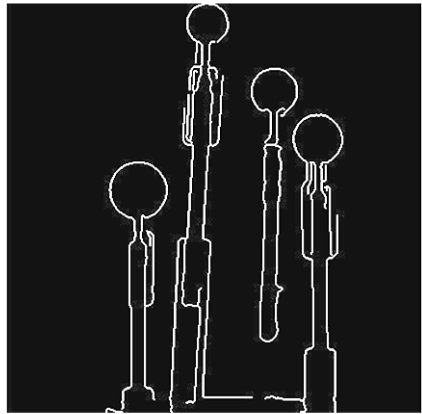

Edges of the spheres in the calibration phantom

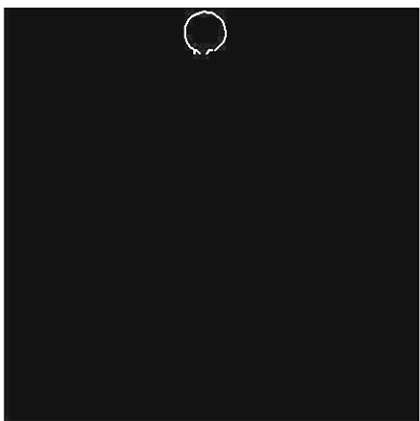

Edges of the Sphere 1 identified in the calibration phantom

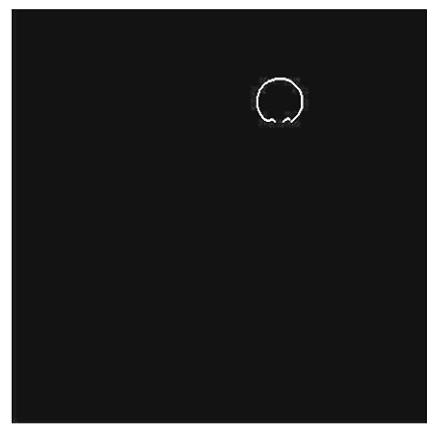

Edges of the Sphere 2 identified in the calibration phantom

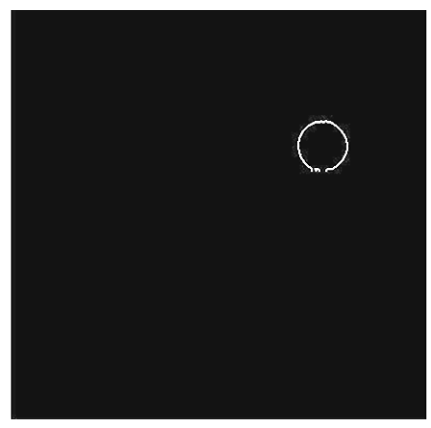

Edges of the Sphere 3 identified in the calibration phantom

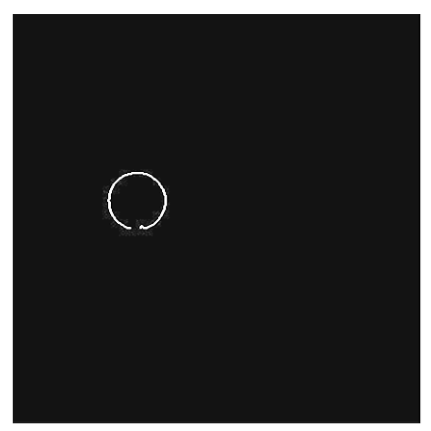

Edges of the Sphere 4 identified in the calibration phantom

Figure 23: Edges of the four spheres identified in the reference image

5. Find the centroid of the sphere 1 as Centroid $X_{-} S p_{1}$ and find the respective $Y$ coordinate as $Y_{-} S p_{1}$

6. Calculate the distance of the centroids of all the spheres from $X_{0}$ and save it as

$$
a_{-} S p 1=\text { Centroid }_{-} X_{-} S p 1-X_{0}
$$


7. Repeat steps 5 and 6 for the remaining spheres such that $a_{-} S p_{2}, a_{-} S p_{3}, a_{-} S p_{4}$ are obtained for the four spheres

8. Repeat steps 2 to 7 for image 1 and image 2 such that $a_{1} \_S p_{1}, a_{1} \_S p_{2}, a_{1} \_S p_{3}, a_{1} \_S p_{4}$ for image 1 and $a_{2}-S p_{1}, a_{2} S p_{2}, a_{2}-S p_{3}, a_{2}-S p_{4}$ for image 2 are calculated

9. Calculate radius $R_{-} S p_{1}$ for sphere1 in the reference image as below where $R_{-} S p_{1}$ and $\theta_{-} S p_{1}$ are unknown

$$
R_{-} S p_{1}=\frac{a_{-} S p_{1}}{\cos \left(\theta_{-} S p_{1}\right)}
$$

10. Calculate radius $R \_S p_{1}$ for sphere 1 in image 1 as below where $\theta_{1} S p_{1}$ is known and $R_{-} S p_{1}$ is unknown

$$
R_{-} S p_{1}=\frac{a 1 \_S p_{1}}{\cos \left(\theta \_S p_{1}+\theta 1 \_S p_{1}\right)}
$$

11. Calculate radius $R_{-} S p_{1}$ for sphere 1 in image 2 as below where $\theta_{2} S p_{1}$ is known and $R \_S p_{1}$ is unknown

$$
R_{-} S p_{1}=\frac{a 2 \_S p_{1}}{\cos \left(\theta \_S p_{1}+\theta 2 \_S p_{1}\right)}
$$

12. From equation (18) and equation (19) solve for $\theta_{-} S p_{1}$ as below

$$
\frac{a 1 \_S p_{1}}{\cos \left(\theta \_S p_{1}+\theta 1 \_S p_{1}\right)}=\frac{a 2 \_S p_{1}}{\cos \left(\theta \_S p_{1}+\theta 2 \_S p_{1}\right)}
$$




$$
\theta_{-} S p_{1}=\arctan \left(\frac{\left(\left(a 1_{-} S p_{1} * \cos \left(\theta 2_{-} S p_{1}\right)\right)-\left(a 2_{-} S p_{1} * \cos \left(\theta 1_{-} S p_{1}\right)\right)\right)}{\left(\underline{\left.\left(a 1_{-} S p_{1} * \sin \left(\theta 2_{-} S p_{1}\right)\right)-\left(a 2_{-} S p_{1} * \sin \left(\theta 1 \_S p_{1}\right)\right)\right)}\right)}\right)
$$

13. From equation (19) calculate $R_{-} S p_{1}$ using the value of $\theta_{-} S p_{1}$ obtained from equation (21)

14. Find $\mathrm{X}, \mathrm{Y}$ and $\mathrm{Z}$ coordinates of the centroid for sphere 1 as below

$$
\begin{gathered}
X_{o p_{-} S p_{1}}=R_{-} S p_{1} * \cos \left(\theta_{-} S p_{1}\right)+X_{0} \\
Y_{o p_{-} S p_{1}}=Y_{-} S p_{1} \\
Z_{o p_{-} S p_{1}}=\left(R_{-} S p_{1} * \sin \left(\theta_{-} S p_{1}\right)\right)+\frac{r_{1}}{2}
\end{gathered}
$$

15. Repeat steps 9 to 14 for the remaining three spheres to calculate $\mathrm{X}, \mathrm{Y}$ and $\mathrm{Z}$ coordinates

\subsubsection{Homogeneous Coordinate Transformation}

\subsubsection{Theory}

- A vector is represented as a matrix

$$
\vec{p}=\left\lfloor\begin{array}{l}
a_{x} \\
b_{y} \\
c_{z}
\end{array}\right\rfloor
$$

- Homogeneous matrix is represented as below

$$
\left[\begin{array}{cccc}
a_{x} & b_{x} & c_{x} & d_{x} \\
a_{y} & b_{y} & c_{y} & d_{y} \\
a_{z} & b_{z} & c_{z} & d_{z} \\
0_{x} & 0 & 0 & 1
\end{array} \mid\right.
$$


- In the above matrix the following part of the matrix represents the rotations about $\mathrm{X}, \mathrm{Y}$ and $\mathrm{Z}$ axes

$$
\left\lfloor\begin{array}{lll}
a_{x} & b_{x} & c_{x} \\
a_{y} & b_{y} & c_{y} \\
a_{z} & b_{z} & c_{z}
\end{array}\right\rfloor
$$

- The last column of the homogeneous matrix represents the translation

$$
\left\lfloor\begin{array}{l}
d_{x} \\
d_{y} \\
d_{z}
\end{array}\right\rfloor
$$

- The fourth row is added to combine the rotations and translations into a single $4 * 4$ matrix.

\subsection{Scale Transformations}

- Non Uniform Scaling scales the object along $\mathrm{X}, \mathrm{Y}$ and $\mathrm{Z}$ axes by $\mathrm{s}_{\mathrm{x}}, \mathrm{s}_{\mathrm{y}}$ and $\mathrm{s}_{\mathrm{z}}$ factor respectively.

$$
S_{n}(s)=\left[\begin{array}{cccc}
s_{x} & 0 & 0 & 0 \\
0 & s_{y} & 0 & 0 \\
0 & 0 & s_{z} & 0 \\
0 & 0 & 0 & 1
\end{array}\right]
$$

- The entire object is scaled by a factor of $\mathrm{S}$ by using a uniform scaling matrix

$$
S_{u}(s)=\left[\begin{array}{cccc}
s & 0 & 0 & 0 \\
0 & s & 0 & 0 \\
0 & 0 & s & 0 \\
0 & 0 & 0 & 1
\end{array}\right]
$$




\subsection{Rotation Matrices}

$$
\begin{aligned}
& R_{x}(\theta)=\left[\begin{array}{cccc}
1 & 0 & 0 & 0 \\
0 & \cos \theta & -\sin \theta & 0 \\
0 & \sin \theta & \cos \theta & 0 \\
0 & 0 & 0 & 1
\end{array}\right] \\
& R_{y}(\theta)=\left[\begin{array}{cccc}
\cos \theta & 0 & \sin \theta & 0 \\
0 & 1 & 0 & 0 \\
-\sin \theta & 0 & \cos \theta & 0 \\
0 & 0 & 0 & 1
\end{array}\right] \\
& R_{z}(\theta)=\left[\begin{array}{cccc}
\cos \theta & -\sin \theta & 0 & 0 \\
\sin \theta & \cos \theta & 0 & 0 \\
0 & 0 & 1 & 0 \\
0 & 0 & 0 & 1
\end{array}\right]
\end{aligned}
$$

\subsection{Translation Matrix}

A translation matrix that translates an object by vector $r$ is as shown below

$$
T(r)=\left[\begin{array}{cccc}
1 & 0 & 0 & r_{x} \\
0 & 1 & 0 & r_{y} \\
0 & 0 & 1 & r_{z} \\
0 & 0 & 0 & 1
\end{array}\right]
$$

\subsection{General Combination Matrix}

A sequence of translations, scalings, rotations and shears can be combined to form a single matrix. The order in which the rotations, scaling and translations are done is important. All the matrices explained above have the last row of the form [ $\left[\begin{array}{llll}0 & 0 & 0 & 1\end{array}\right]$. When any two matrices of this form are multiplied the resulting matrix will also be of the same form $\left[\begin{array}{llll}0 & 0 & 0 & 1\end{array}\right]$. The single matrix is as follows:

$$
V^{\prime}=M V
$$




$$
\begin{aligned}
& {\left[\begin{array}{c}
V_{x}{ }^{\prime} \\
V_{y}^{\prime} \\
V_{z}^{\prime} \\
1
\end{array}\right]=\left[\begin{array}{cccc}
a_{1} & b_{1} & c_{1} & d_{1} \\
a_{2} & b_{2} & c_{2} & d_{2} \\
a_{3} & b_{3} & c_{3} & d_{3} \\
0 & 0 & 0 & 1
\end{array}\right]\left[\begin{array}{c}
V_{x} \\
V_{y} \\
V_{z} \\
1
\end{array}\right]} \\
& V_{x}^{\prime}=a_{1} V_{x}+b_{1} V_{y}+c_{1} V_{z}+d_{1} \\
& V_{y}{ }^{\prime}=a_{2} V_{x}+b_{2} V_{y}+c_{2} V_{z}+d_{2} \\
& V_{z}^{\prime}=a_{3} V_{x}+b_{3} V_{y}+c_{3} V_{z}+d_{3} \\
& 1=0 V_{x}+0 V_{y}+0 V_{z}+1
\end{aligned}
$$

\subsubsection{Implementation to the current problem}

The centroids of the four spheres are identified in the optical and PET modalities. The optical coordinates of the centroids of the four spheres are obtained from the calibration procedure as $\left(X_{o p 1}, Y_{o p 1}, Z_{o p 1}\right),\left(X_{o p 2}, Y_{o p 2}, Z_{o p 2}\right),\left(X_{o p 3}, Y_{o p 3}, Z_{o p 3}\right),\left(X_{o p 4}, Y_{o p 4}, Z_{o p 4}\right)$. The PET coordinates of the centroids of the spheres are $\left(X_{p 1}, Y_{p 1}, Z_{p 1}\right),\left(X_{p 2}, Y_{p 2}, Z_{p 2}\right)$, $\left(X_{p 3}, Y_{p 3}, Z_{p 3}\right),\left(X_{p 4}, Y_{p 4}, Z_{p 4}\right)$. Optical coordinates are represented by V, PET coordinates are represented by $V^{\prime}$ and the combination matrix by $\mathrm{M}$ as shown in equation(25). The combination matrix is calculated from the coordinates of the four spheres obtained from both the modalities using the Gaussian elimination method. The procedure followed to find the matrix $\mathrm{M}$ is explained in this section:

$$
\begin{aligned}
& \left.\left\lfloor\begin{array}{c}
X_{o p 1} \\
Y_{o p 1} \\
Z_{o p 1} \\
1
\end{array}\right\rfloor=\left\lfloor\begin{array}{cccc}
a_{1} & b_{1} & c_{1} & d_{1} \\
a_{2} & b_{2} & c_{2} & d_{2} \\
a_{3} & b_{3} & c_{3} & d_{3} \\
0 & 0 & 0 & 1
\end{array}\right\rfloor \mid \begin{array}{c}
X_{p 1} \\
Y_{p 1} \\
Z_{p 1} \\
1
\end{array}\right\rfloor \\
& \left.\left\lfloor\begin{array}{c}
X_{o p 2} \\
Y_{o p 2} \\
Z_{o p 2} \\
1
\end{array}\right\rfloor=\left\lfloor\begin{array}{llll}
a_{1} & b_{1} & c_{1} & d_{1} \\
a_{2} & b_{2} & c_{2} & d_{2} \\
a_{3} & b_{3} & c_{3} & d_{3} \\
0 & 0 & 0 & 1
\end{array}\right\rfloor \mid \begin{array}{c}
X_{p 2} \\
Y_{p 2} \\
Z_{p 2} \\
1
\end{array}\right\rfloor
\end{aligned}
$$




$$
\begin{aligned}
& \left.\left\lfloor\begin{array}{c}
X_{o p 3} \\
Y_{o p 3} \\
Z_{o p 3} \\
1
\end{array}\right\rfloor=\left\lfloor\begin{array}{llll}
a_{1} & b_{1} & c_{1} & d_{1} \\
a_{2} & b_{2} & c_{2} & d_{2} \\
a_{3} & b_{3} & c_{3} & d_{3} \\
0 & 0 & 0 & 1
\end{array}\right\rfloor \mid \begin{array}{c}
X_{p 3} \\
Y_{p 3} \\
Z_{p 3} \\
1
\end{array}\right\rfloor \\
& \left.\left\lfloor\begin{array}{c}
X_{o p 4} \\
Y_{o p 4} \\
Z_{o p 4} \\
1
\end{array}\right\rfloor=\left\lfloor\begin{array}{llll}
a_{1} & b_{1} & c_{1} & d_{1} \\
a_{2} & b_{2} & c_{2} & d_{2} \\
a_{3} & b_{3} & c_{3} & d_{3} \\
0 & 0 & 0 & 1
\end{array}\right\rfloor \mid \begin{array}{c}
X_{p 4} \\
Y_{p 4} \\
Z_{p 4} \\
1
\end{array}\right\rfloor
\end{aligned}
$$

From equations (27), (28), (29) and (30)

$$
\begin{aligned}
& X_{o p 1}=a_{1} X_{p 1}+b_{1} Y_{p 1}+c_{1} Z_{p 1}+d_{1} \\
& Y_{o p 1}=a_{2} X_{p 1}+b_{2} Y_{p 1}+c_{2} Z_{p 1}+d_{2} \\
& Z_{o p 1}=a_{3} X_{p 1}+b_{3} Y_{p 1}+c_{3} Z_{p 1}+d_{3} \\
& X_{o p 2}=a_{1} X_{p 2}+b_{1} Y_{p 2}+c_{1} Z_{p 2}+d_{1} \\
& Y_{o p 2}=a_{2} X_{p 2}+b_{2} Y_{p 2}+c_{2} Z_{p 2}+d_{2} \\
& Z_{o p 2}=a_{3} X_{p 2}+b_{3} Y_{p 2}+c_{3} Z_{p 2}+d_{3} \\
& X_{o p 3}=a_{1} X_{p 3}+b_{1} Y_{p 3}+c_{1} Z_{p 3}+d_{1} \\
& Y_{o p 3}=a_{2} X_{p 3}+b_{2} Y_{p 3}+c_{2} Z_{p 3}+d_{2} \\
& Z_{o p 3}=a_{3} X_{p 3}+b_{3} Y_{p 3}+c_{3} Z_{p 3}+d_{3} \\
& X_{o p 4}=a_{1} X_{p 4}+b_{1} Y_{p 4}+c_{1} Z_{p 4}+d_{1} \\
& y_{o p 4}=a_{2} X_{p 4}+b_{2} Y_{p 4}+c_{2} Z_{p 4}+d_{2} \\
& Z_{o p 4}=a_{3} X_{p 4}+b_{3} Y_{p 4}+c_{3} Z_{p 4}+d_{3}
\end{aligned}
$$


Equations 7, 10, 13, 16 can be written as

$$
\left[\begin{array}{l}
X_{o p 1} \\
X_{o p 2} \\
X_{o p 3} \\
X_{o p 4}
\end{array}\right]=\left[\begin{array}{llll}
X_{p 1} & Y_{p 1} & Z_{p 1} & 1 \\
X_{p 2} & Y_{p 2} & Z_{p 2} & 1 \\
X_{p 3} & Y_{p 3} & Z_{p 3} & 1 \\
X_{p 4} & Y_{p 4} & Z_{p 4} & 1
\end{array}\right]\left[\begin{array}{l}
a_{1} \\
b_{1} \\
c_{1} \\
d_{1}
\end{array}\right]
$$

Similarly equations $8,11,14,17$ can be written as

$$
\left[\begin{array}{l}
Y_{o p 1} \\
Y_{o p 2} \\
Y_{o p 3} \\
Y_{o p 4}
\end{array}\right]=\left[\begin{array}{llll}
X_{p 1} & Y_{p 1} & Z_{p 1} & 1 \\
X_{p 2} & Y_{p 2} & Z_{p 2} & 1 \\
X_{p 3} & Y_{p 3} & Z_{p 3} & 1 \\
X_{p 4} & Y_{p 4} & Z_{p 4} & 1
\end{array}\right]\left[\begin{array}{l}
a_{2} \\
b_{2} \\
c_{2} \\
d_{2}
\end{array}\right]
$$

Equations 9, 12, 16, 19 can be written as

$$
\left[\begin{array}{c}
Z_{o p 1} \\
Z_{o p 2} \\
Z_{o p 3} \\
Z_{o p 4}
\end{array}\right]=\left[\begin{array}{llll}
X_{p 1} & Y_{p 1} & Z_{p 1} & 1 \\
X_{p 2} & Y_{p 2} & Z_{p 2} & 1 \\
X_{p 3} & Y_{p 3} & Z_{p 3} & 1 \\
X_{p 4} & Y_{p 4} & Z_{p 4} & 1
\end{array}\right]\left[\begin{array}{l}
a_{3} \\
b_{3} \\
c_{3} \\
d_{3}
\end{array}\right]
$$

Let

$$
\begin{gathered}
P E T=\left[\begin{array}{llll}
X_{p 1} & Y_{p 1} & Z_{p 1} & 1 \\
X_{p 2} & Y_{p 2} & Z_{p 2} & 1 \\
X_{p 3} & Y_{p 3} & Z_{p 3} & 1 \\
X_{p 4} & Y_{p 4} & Z_{p 4} & 1
\end{array}\right] \\
\text { optical_X }=\left\lfloor\begin{array}{l}
X_{o p 1} \\
X_{o p 2} \\
X_{o p 3} \\
X_{o p 4}
\end{array}\right\rfloor
\end{gathered}
$$




$$
\begin{aligned}
\text { optical_Y } & =\left\lfloor\begin{array}{l}
Y_{o p 1} \\
Y_{o p 2} \\
Y_{o p 3} \\
Y_{o p 4}
\end{array}\right\rfloor \\
\text { optical_Z } & =\left\lfloor\begin{array}{l}
Z_{o p 1} \\
Z_{o p 2} \\
Z_{o p 3} \\
Z_{o p 4}
\end{array}\right\rfloor
\end{aligned}
$$

$$
\text { and } \quad A_{1}=\left\lfloor\begin{array}{l}
a_{1} \\
b_{1} \\
c_{1} \\
d_{1}
\end{array}\right\rfloor, \quad A_{2}=\left\lfloor\begin{array}{l}
a_{2} \\
b_{2} \\
c_{2} \\
d_{2}
\end{array}\right\rfloor, \quad A_{3}=\left\lfloor\begin{array}{l}
a_{3} \\
b_{3} \\
c_{3} \\
d_{3}
\end{array}\right\rfloor
$$

From the above equations the combination matrix can be calculated following the Gaussian elimination method as explained below.

$$
\begin{aligned}
& A_{1}=\frac{P E T}{\text { Optical_X }_{-}} \\
& A_{2}=\frac{\text { PET }}{\text { Optical_Y }_{-}} \\
& A_{3}=\frac{\text { PET }}{\text { Optical_Z }} \\
& A_{4}=\left[\begin{array}{llll}
0 & 0 & 0 & 1
\end{array}\right]
\end{aligned}
$$

Therefore combination matrix

$$
T=\left[\begin{array}{c}
A_{1}{ }^{\prime} \\
A_{2}{ }^{\prime} \\
A_{3}{ }^{\prime} \\
A_{4}{ }^{\prime}
\end{array}\right] \text { Which means? }
$$




$$
T=\left[\begin{array}{cccc}
a_{1} & b_{1} & c_{1} & d_{1} \\
a_{2} & b_{2} & c_{2} & d_{2} \\
a_{3} & b_{3} & c_{3} & d_{3} \\
0 & 0 & 0 & 1
\end{array} \mid\right.
$$

\subsubsection{Image Fusion with Phantom}

The PET slices of the phantom are transformed by the combination matrix shown in equation 19. PET slices show the radioactivity identified in the phantom as a white blob on black background.

After transformation, the coordinates of the PET slices are obtained with respect to the optical coordinate system. The PET and optical slices with the same Y coordinates i.e. the same slices are identified. The respective $\mathrm{X}$ and $\mathrm{Z}$ coordinates for a particular image of a slice are filled with the pixel values of 1

1. Initialize

- $\quad$ nimg_PET $=60$

- Last_Slice, identified in the optical reconstruction as the last slice

2. FOR 1: nimg_PET

- Read the image

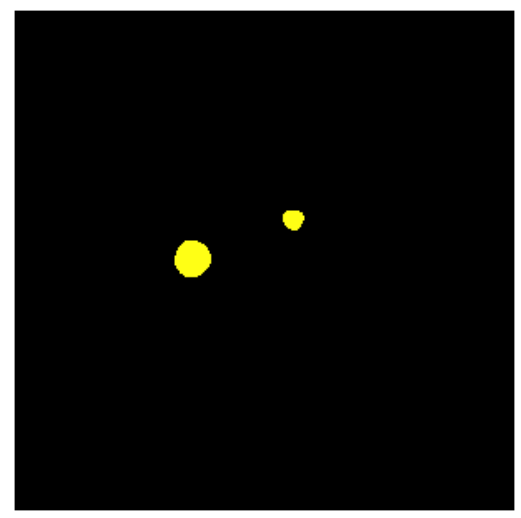

Figure 24: PET slice

- Change the image to binary image

- Initialize PET_X=[ ] as an empty matrix

- Identify the $X$ and $Z$ coordinates of the activity in the slices as PET_X and PET_Z

PET_X=column pixel coordinate 
PET_Z=row pixel coordinate

- IF PET_X is empty

Continue;

End

- Save PET_X, PET_Y and PET_Z coordinates in PET_Coords where PET_Y is the slice number

- Multiply PET_Coords by the matrix T as in equation (46).

$$
\text { PET_Trans_Coords }=\text { PET_Coords.*T }
$$

- Save the PET_Trans_Coords of all the images where activity is present as PET_coordmatrix

END FOR

- Identify the corresponding optical slices and fill the pixels of respective IMG1 with the PET_Trans_Coords

○ FOR $\mathrm{j}=1$ to length (PET_Trans_Coords)

IMG1 (PET_Trans_Coords (j, 3), PET_Trans_Coords $(\mathrm{j}, 1))=1$

\section{END FOR}

- A sample of merged slice is shown in Figure 25. 


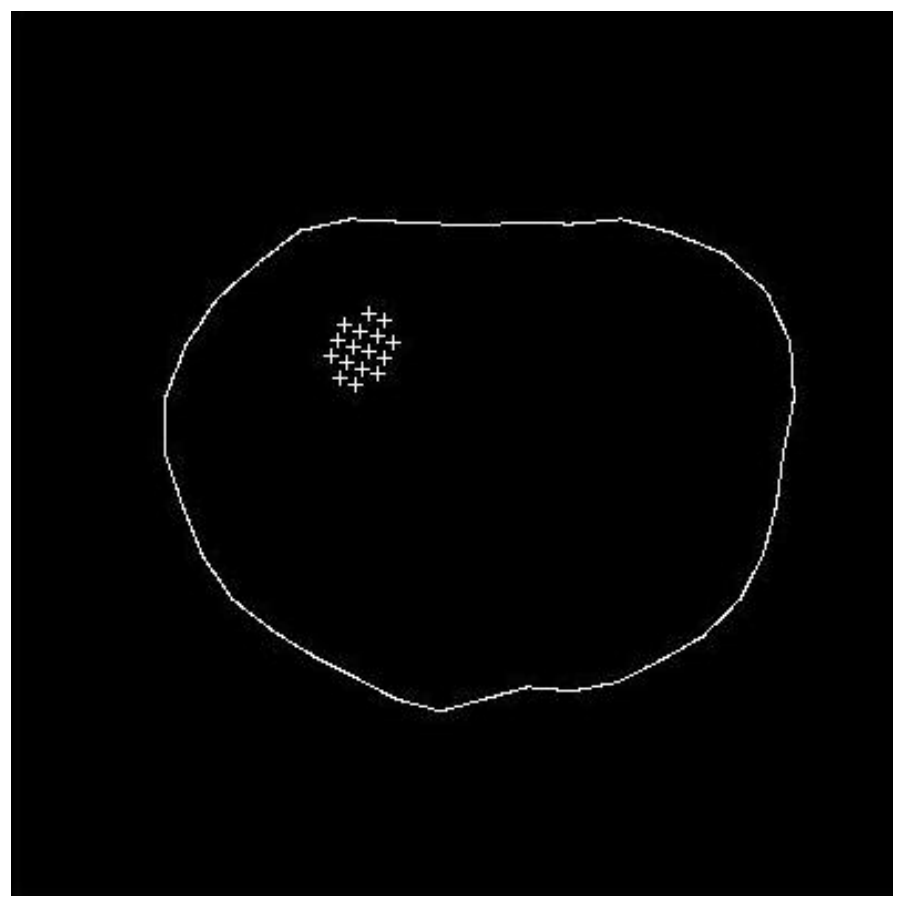

Figure 25: Merged slice at $9 \mathrm{~mm}$

\subsection{Margin Evaluation}

Stitches are placed at 12 o' clock reference and 3 o' clock reference which are called as superior and lateral as per the margin assessment method. Margins are evaluated in the superior, lateral, medial, anterior and posterior directions in a standard type of margin assessment method which is identified by 6 different colors as shown in Figure 27.

In the current scenario, the points on the radioactive blob were identified in the superior, lateral, medial and inferior directions. The distance between these points and the points on the edges is measured and checked if it is less than $5 \mathrm{~mm}$ or greater than $5 \mathrm{~mm}$. The distance between these points is called the margin. If the margin is less than $5 \mathrm{~mm}$ then it is considered a positive margin and if it is greater than $5 \mathrm{~mm}$ then it is considered a negative margin. Figure 26 shows the image of an excised breast tissue with the margins marked in different colors. The margins are assessed in the specified directions. Let the points on the blob for a slice be represented by $(\mathrm{x}, \mathrm{z})$ and the points on the edge be represented by $(\mathrm{X}, \mathrm{Z})$. The distance between these points is calculated as below:

1. $M \arg$ in $=\sqrt{\left((X-x)^{2}+(Z-z)^{2}\right)}$ 
2. If Margin $>5 \mathrm{~mm}$

Display ('Negative Margins')

Else if Margin $<5 \mathrm{~mm}$

Display ('Positive Margins')

End

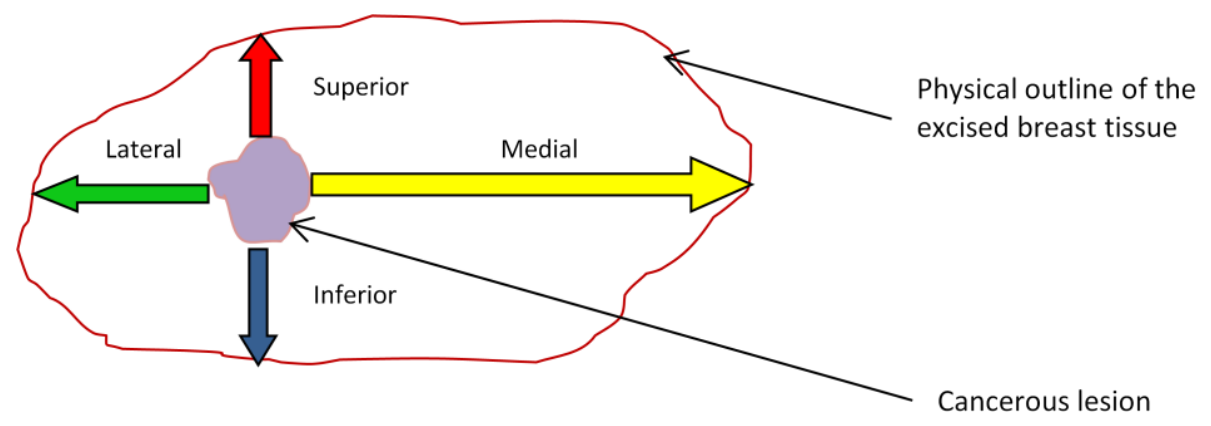

Figure 26: Margins in an excised breast tissue 


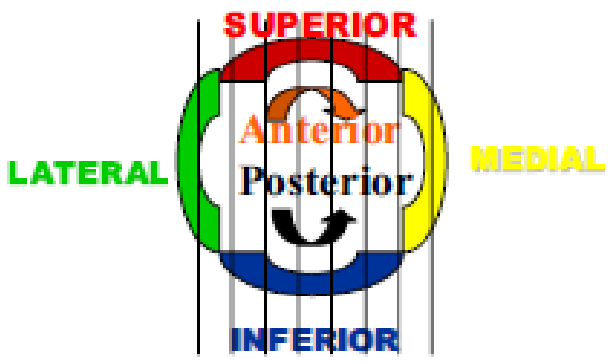

Figure 27: Margin Assessment Method (7) 


\section{Results and Discussion}

This chapter includes the experiments performed to ensure the correctness and accuracy of the algorithm. Various phantoms of different shapes were used which were similar to the real excised breast tissue. Different calibration procedures were followed and the corresponding results are discussed here.

\subsection{Calibration Procedures}

\subsubsection{Pixel to Millimeter Calibration}

A squash ball of known dimensions was used to perform the millimeter to pixel calibration. The pixel to mm conversion factor was found to be the same in both horizontal and vertical directions.

$$
\text { Pixel2mm_Factor }(\text { Vertical })=\text { Pixel2mm_Factor }(\text { Horizontal })
$$

The Pixel2mm_Factor was found to be 4.8 pixels for $1 \mathrm{~mm}$ for that particular focal length of the lens used and the camera position. The Pixel2mm_Factor varies with the change in focal length and the position of the camera.

\subsubsection{Axis of rotation Calibration}

The images were cropped to $400 * 400$ resolution and $\mathrm{X}_{0}$ and $\mathrm{Y}_{0}$ were identified as $(215,395)$ in the image coordinate system using the procedure explained in the section 3.4.2.2.

\subsubsection{Calibration for Image Fusion}

Figure 22 shows the calibration phantom used to find the homogeneous matrix parameters. The procedure is explained in section 3.5. Five sets of camera images were taken to find the centroids of the four spheres in the optical system. Each set consists of three images. An image taken at 20 degrees of the turntable was considered as the reference image for all the sets. The images in which all the four spheres were visible were considered for the analysis. The sets of images were as follows: 
Set 1: Reference image at 0 degrees, Image 1 at 40 degrees and Image 2 at 90 degrees

Set 2: Reference image at 0 degrees, Image 1 at 130 degrees and Image 2 at 220 degrees

Set 3: Reference image at 0 degrees, Image 1 at 270 degrees and Image 2 at 310 degrees

Set 4: Reference image at 0 degrees, Image 1 at 310 degrees, Image 2 at 320 degrees

Set 5: Reference image at 0 degrees, Image 1 at 40 degrees, Image 2 at 320 degrees

Table 4 shows the coordinates of the centroids of the four spheres in optical and PET system. Optical coordinates are calculated using the values given in Table 4 while the PET coordinates are read from the PET slices of the calibration phantom.

Table 4: $(X, Y, Z)$ coordinates of the centroids of spheres in optical and PET coordinate systems

\begin{tabular}{|l|l|l|l|l|l|l|}
\hline & \multicolumn{4}{l|}{ Optical Coordinates } & \multicolumn{4}{l|}{ PET Coordinates } \\
\hline & $\mathrm{X}$ & $\mathrm{Y}$ & $\mathrm{Z}$ & $\mathrm{X}$ & $\mathrm{Y}$ & $\mathrm{Z}$ \\
\hline Sphere 1 & 195.0000 & 29.0000 & 121.9655 & 40.0000 & 11.0000 & 38.0000 \\
\hline Sphere 2 & 259.0000 & 88.0000 & 291.3442 & 22.0000 & 18.0000 & 21.0000 \\
\hline Sphere 3 & 301.0000 & 136.0000 & 160.5544 & 39.0000 & 25.0000 & 23.0000 \\
\hline Sphere 4 & 128.0000 & 186.0000 & 233.5841 & 22.5000 & 31.0000 & 40.0000 \\
\hline
\end{tabular}

The coordinate homogeneous transformation matrix is calculated from the coordinates of the centroids of the four spheres as explained in the section 3.5.2. The matrix shown below is obtained after the calculations and the PET coordinates are transformed using the homogeneous matrix.

$$
\left[\begin{array}{cccc}
3.2298 & 0.1869 & -7.1076 & 333.8395 \\
-0.2989 & 7.5912 & -0.0283 & -41.4722 \\
-7.2029 & -0.4687 & -2.5298 & 511.3700 \\
0 & 0 & 0 & 1
\end{array}\right]
$$




\subsection{Optical 3D Reconstruction and Slice Generation}

\subsubsection{Digital Phantom}

In order to have the proof of concept, an attempt was initially made using a digital phantom. The images of a perfect sphere taken at any interval through the 360 degrees of rotation would be a circle as shown in Figure 28. 35 circles with diameter of 340 pixels in X direction and 339 pixels in $\mathrm{Y}$ direction approximately were taken as input. The algorithm to perform the 3D reconstruction of the surface from the 2 dimensional camera images was applied on this digital phantom. The point cloud obtained from the reconstruction procedure is plotted as shown in Figure 29 using a triangular surface plot in Matlab. Figure 30 shows the reconstructed slices of the digital phantom which are at different heights. The slices are taken from $\mathrm{Y}=\mathrm{Y} 0$ to $\mathrm{Y}=1$, the end of the image space. The estimated diameter of the reconstructed surface was found to be 340 pixels in $\mathrm{X}$ direction, 340 pixels in $\mathrm{Y}$ direction and 341 pixels in $\mathrm{Z}$ direction. The reconstructed diameter is within \pm 1 pixel to the original diameter in all the directions. There are slight distortions present in the slices which are due to the curve fitting procedure followed. These distortions can be minimized by taking more number of images with the degree of rotation being lesser than 10 degrees.

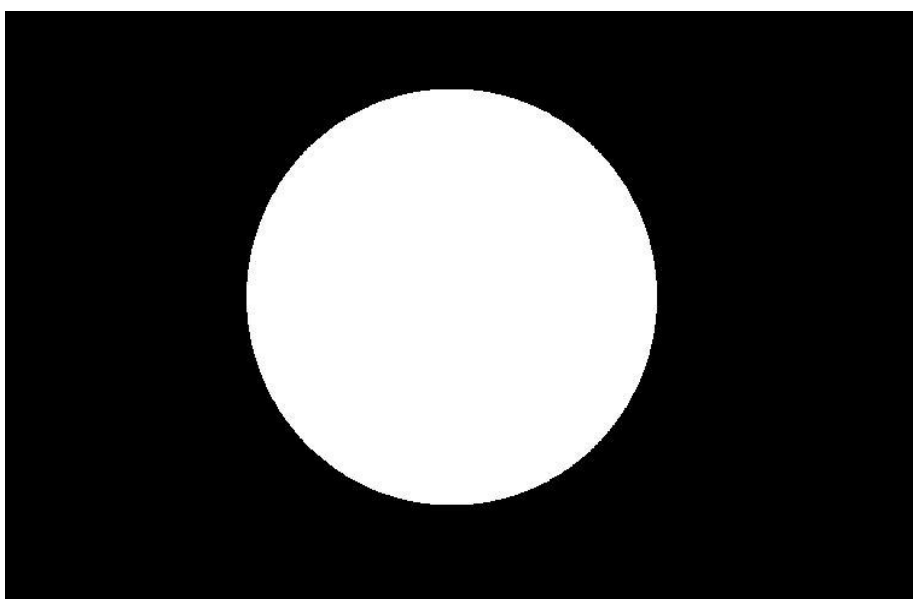

Figure 28: Digital Phantom, Circle of Diameter 84 mm 


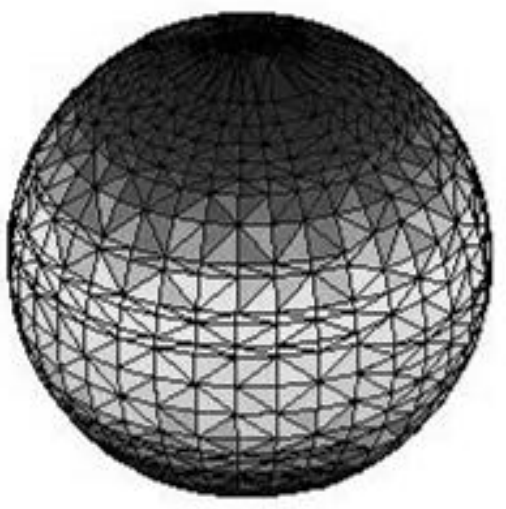

Figure 29: Reconstructed sphere from the point cloud generated from the camera images
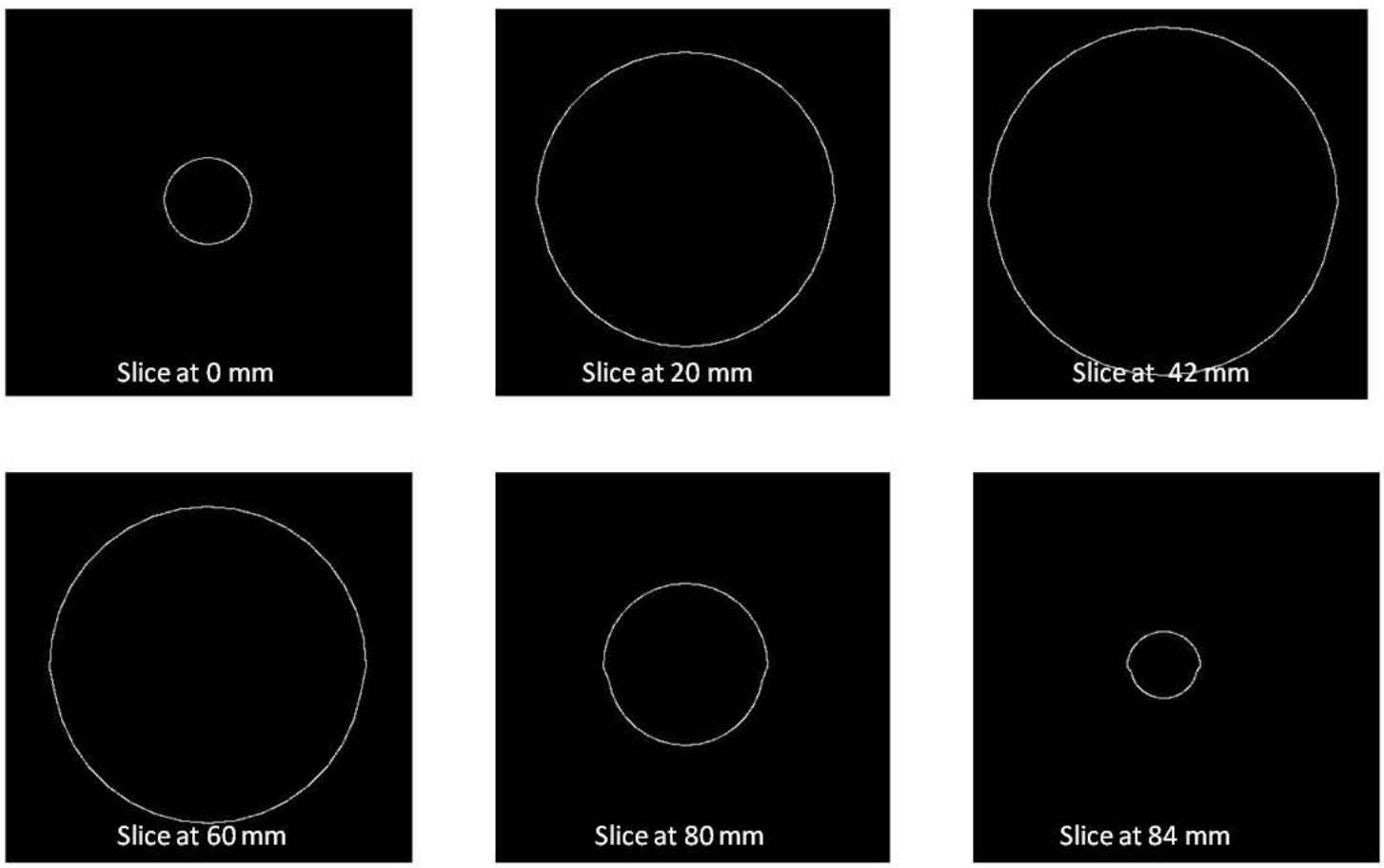

Figure 30: Slices of Digital Phantom 


\subsubsection{Perfect Convex object: Squash Ball}

A squash ball of $39.5 \mathrm{~mm}$ diameter shown in

Figure 31 was taken as a real phantom to check the accuracy of the optical reconstruction. The squash ball was made to rotate from 0 to 350 degrees and images were taken for every 10 degrees interval.

\section{Figure 31: Double Yellow Squash Ball}

Several tests were run using different phantoms with convex surfaces. A squash ball was used as an object with a perfect convex surface. The slices obtained after the reconstruction of the squash ball are shown in Figure 32. Slices are shown at different heights. The diameter of the biggest slice from the reconstructed slices was considered as the estimated diameter of the reconstructed surface. Estimated diameter is $\mathrm{X}=40.5 \mathrm{~mm}$, $\mathrm{Y}=39 \mathrm{~mm}$ and $\mathrm{Z}=40.0 \mathrm{~mm}$. The reconstructed diameter is within $\pm 1 \mathrm{~mm}$ to the original diameter.
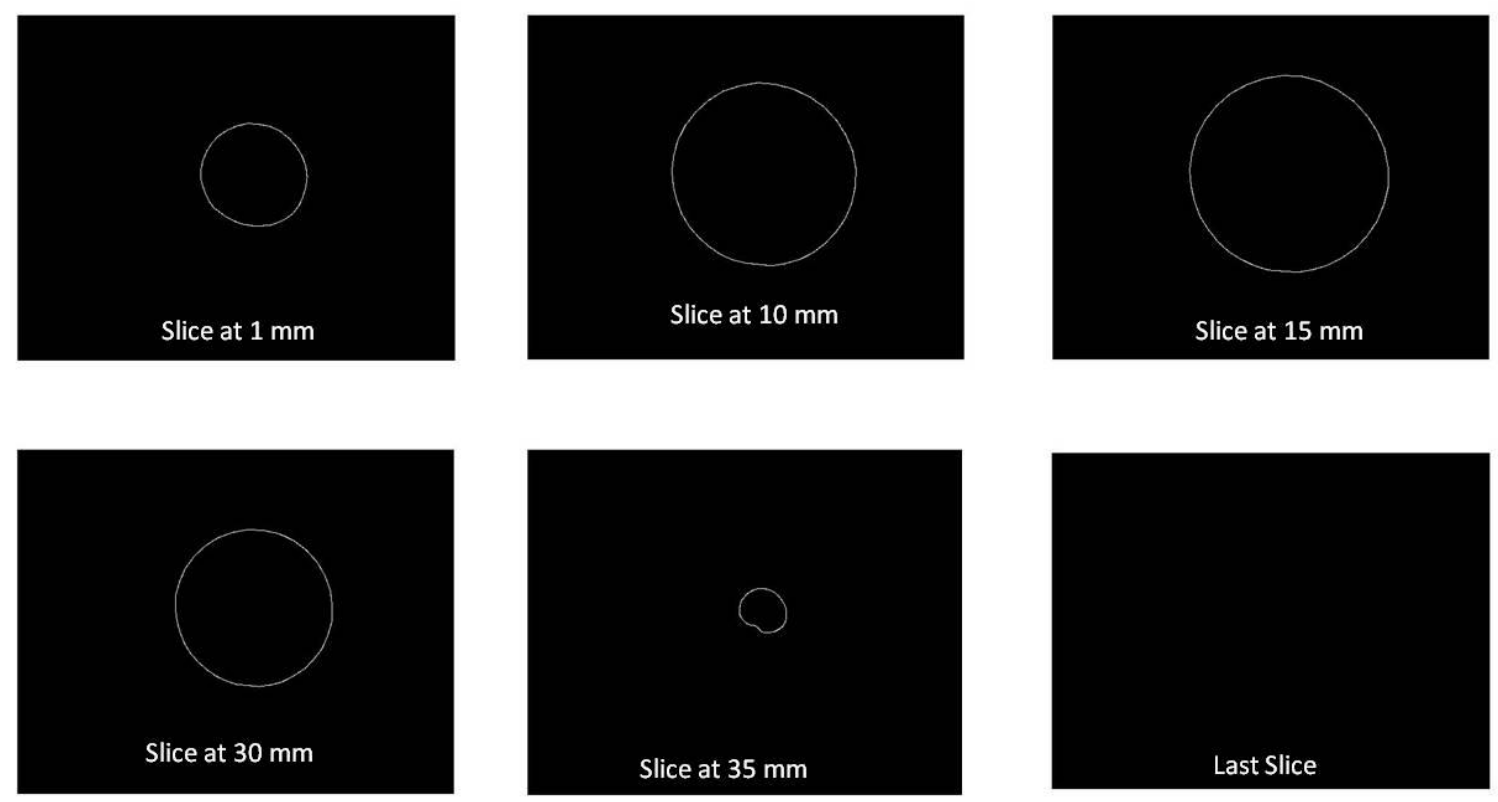

Figure 32: Slices of Squash Ball 


\subsubsection{Irregular Phantoms of different shapes}

Tests with digital phantoms and objects with convex surfaces proved that the system works fine with perfectly convex surfaces. Next an attempt was made to test with phantoms of irregular shapes and sizes. The phantoms were constructed such that they are similar in shape and size to the excised breast tissue. It was taken care that the size of the phantoms was less than 3 inches as the size of the excised breast tissue in lumpectomy would generally be less than 3 inches, according to the oncology surgeon.

\subsubsection{Phantom 1 with Irregular Surface}

Figure 33 shows the phantom that was used to test the reconstruction of an irregular surface. The resolution of the image was $(480 * 621)$ and the AOR was $(317,465)$. The slice at $0 \mathrm{~mm}$ was taken where $\mathrm{Y}=465$ in pixels. As slices start at $\mathrm{Y}=365$ pixels the plot in Figure 34 shows that the points in the bottom row are on the axis in one line while the phantom in Figure 33 looks different in the bottom. Figure 34 was compared with Figure 33 to cross check the accuracy of the reconstruction procedure. It is seen that the front views of the phantom are the same before and after the reconstruction.

Figure 35 shows the slices of the phantom in the top view. The slices are taken from $\mathrm{Y}=\mathrm{Y} 0$ to $\mathrm{Y}=1$, the end of the image space. The size of the slices keeps decreasing from the slice at $0 \mathrm{~mm}$ to the slice at $22 \mathrm{~mm}$ as it is the case with the phantom shown in Figure 33 . The last slice is blank because there is no object present in this part of the image space. 


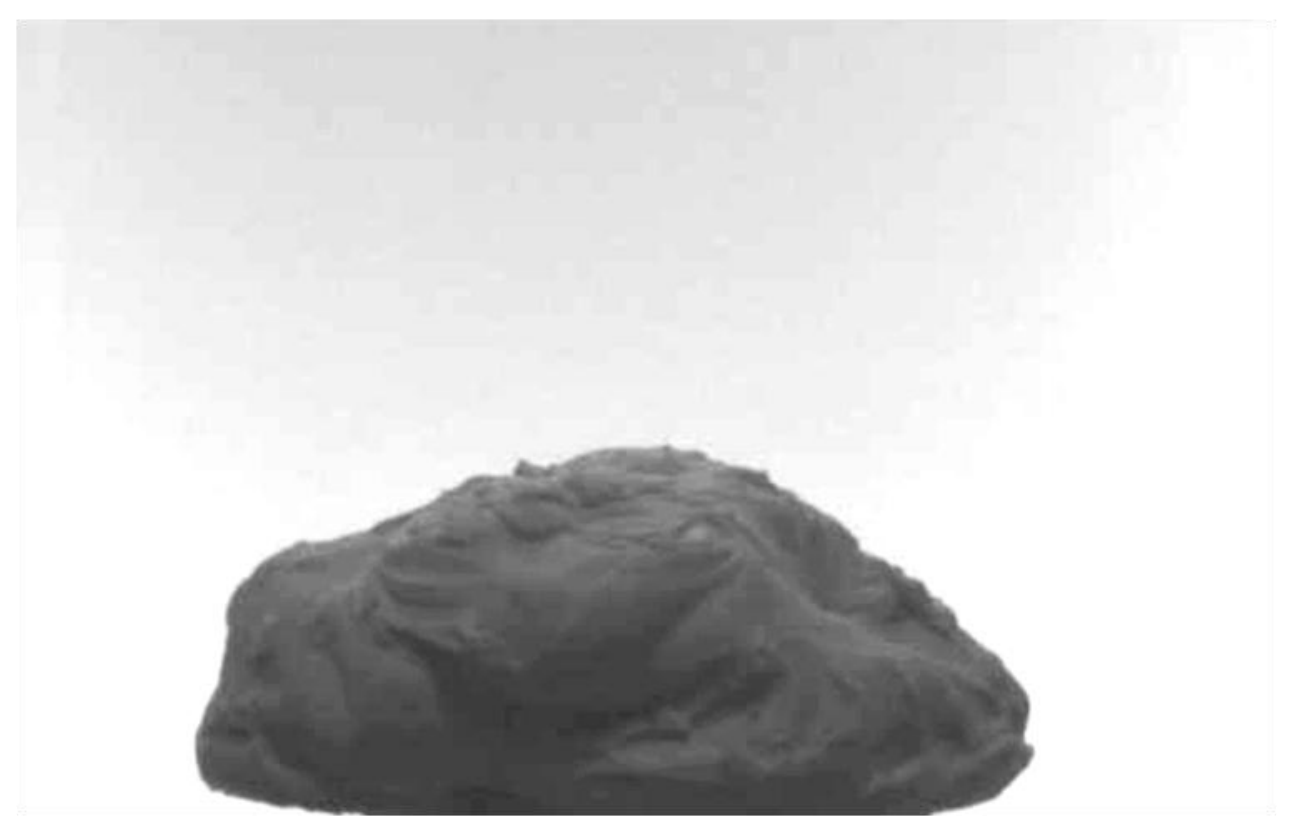

Figure 33: Phantom of Irregular Surface

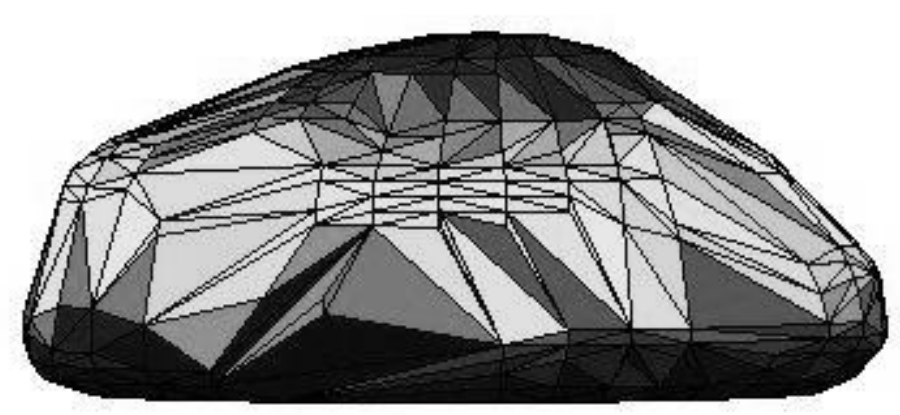

Figure 34: 3D surface plot of the reconstructed Point Cloud 

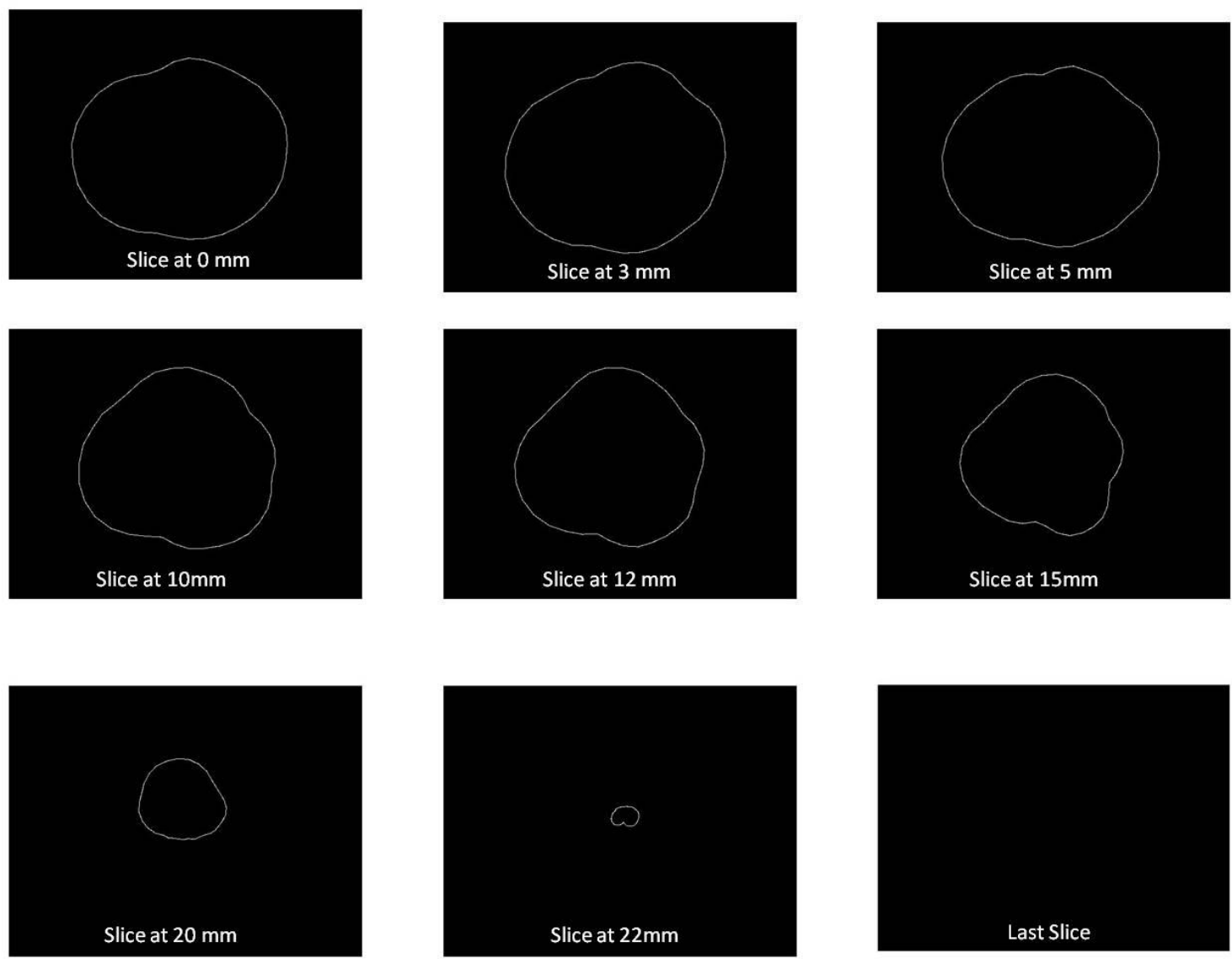

Figure 35: Slices of Irregular Phantom Shown in Figure 33

\subsubsection{Phantom 2 with Irregular Surface}

Figure 36 shows the phantom 2 used to test the reconstruction of irregular phantoms. Figure 37 is the front view of the phantom in Figure 36 as seen by the camera. Comparing Figure 36 with Figure 37 it can be understood that the $3 \mathrm{D}$ reconstructed point cloud is similar to the phantom. There are gaps in between the points because the points are calculated in the steps of one millimeter where each millimeter is equal to 7 pixels. Figure 38 shows the slices which represent the top view of phantom 2 . The slices are generated from $\mathrm{Y}=\mathrm{Y}_{0}$ to $\mathrm{Y}=1$, the end of the image space. 


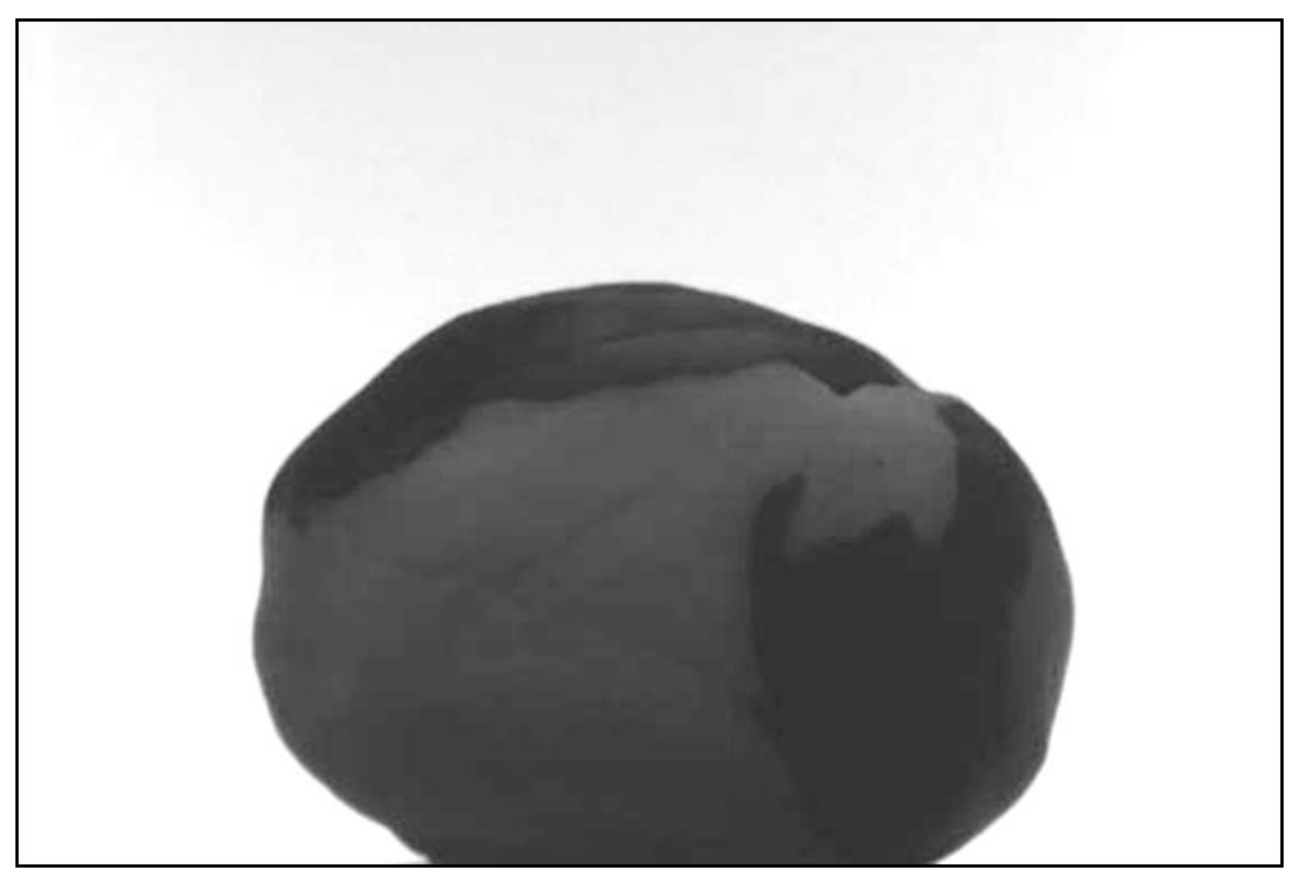

Figure 36: Irregular Phantom 2

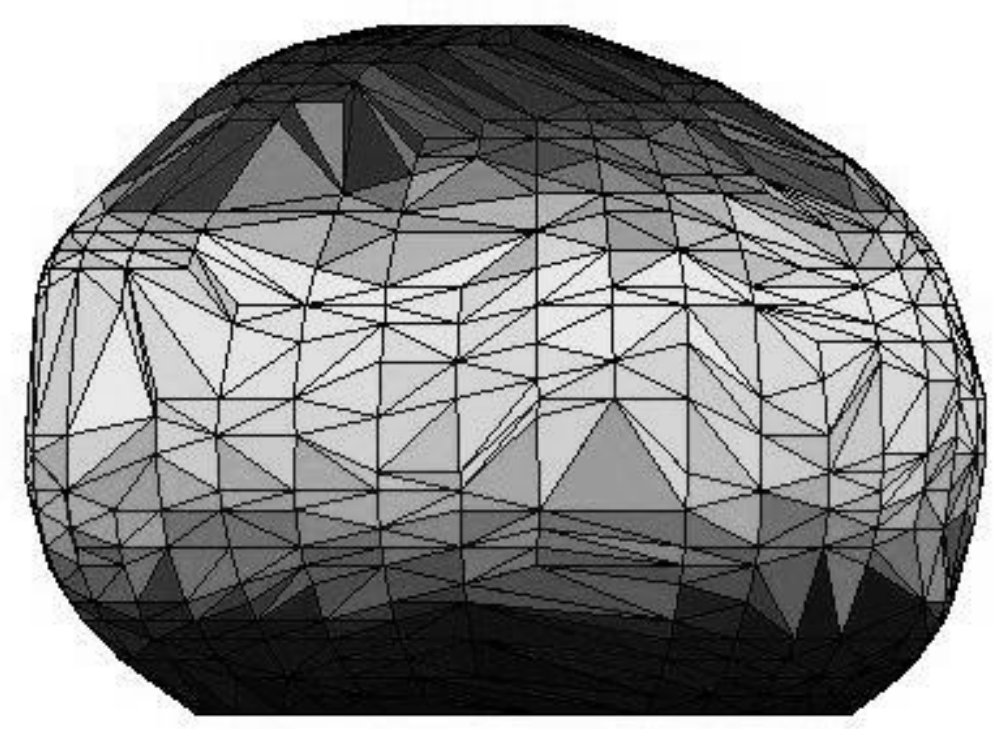

Figure 37: 2D Plot of the 3D reconstructed point Cloud of Phantom 2 

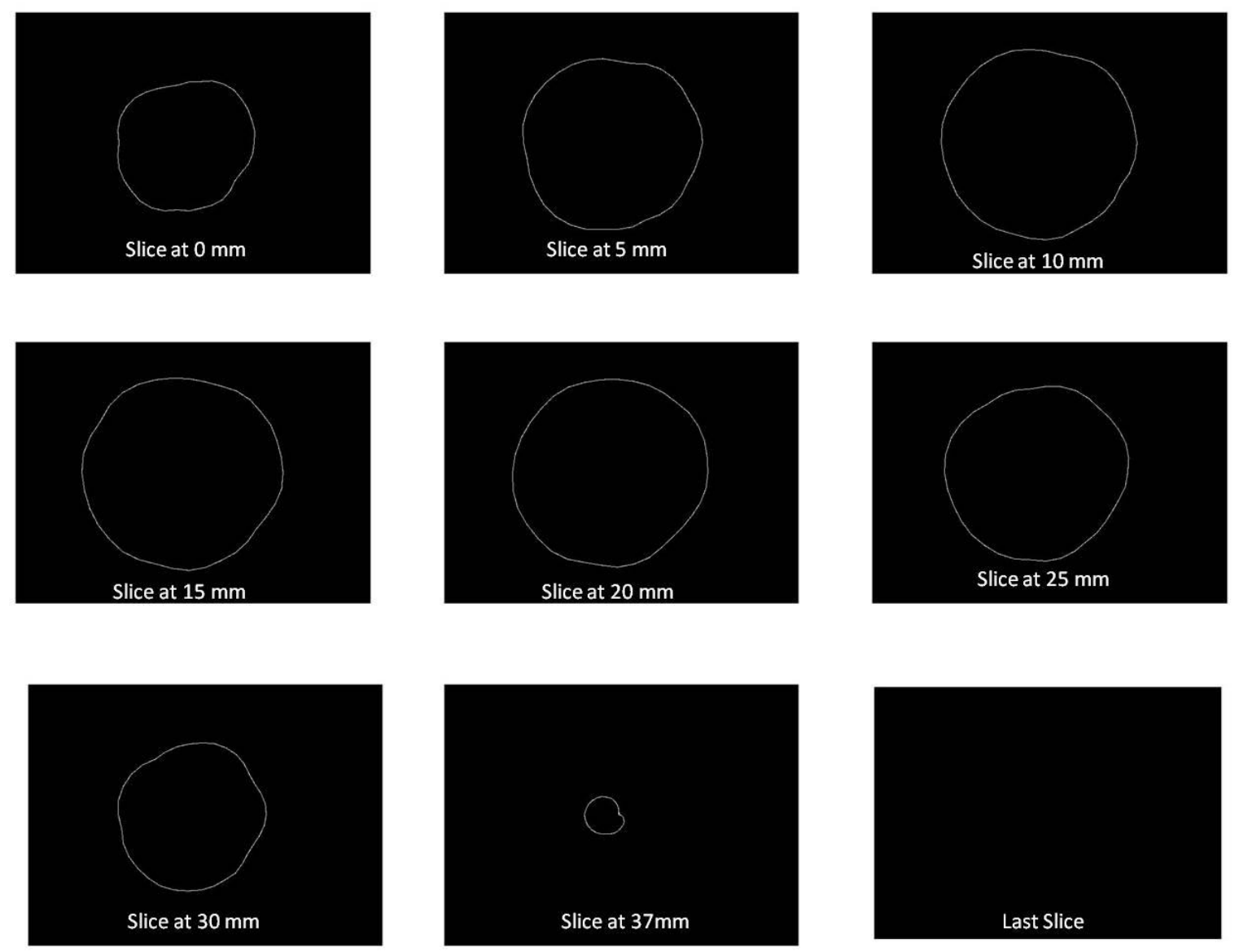

Figure 38: Slices of Phantom 2 with Irregular Surface shown in Figure 36

\subsubsection{Phantom 3 with irregular surface}

Phantom 3 is of a different shape and size when compared to phantoms 1 and 2. Figure 39 shows the phantom 3 used to test the reconstruction of irregular phantoms. Figure 40 is the front view of the phantom 3 as seen by the camera. Comparing Figure 39 with Figure 40 it can be understood that the 3D reconstructed point cloud is similar to the phantom. Figure 41 shows the slices which represent the top view of phantom 2. The slices are generated from $Y=Y_{0}$ to $Y=1$, end of the image space. 


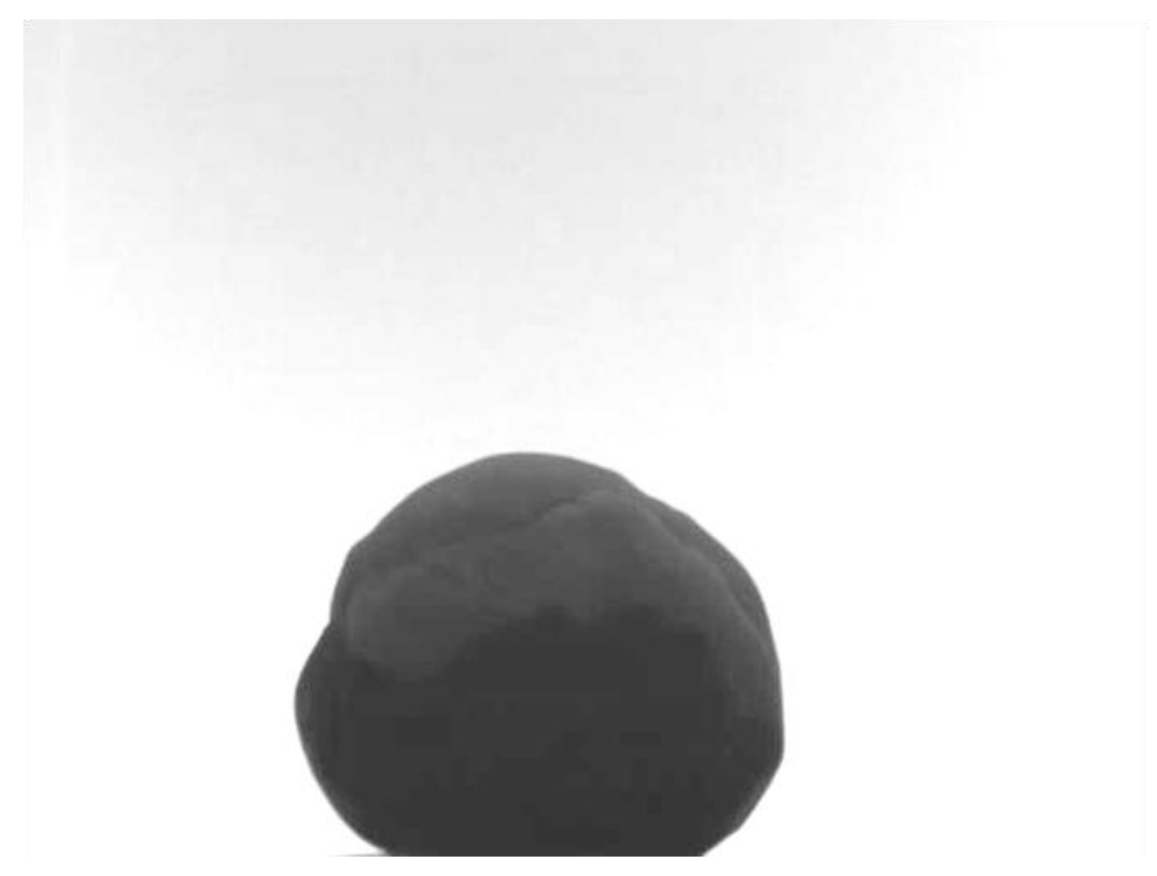

Figure 39: Phantom 3 with Irregular Surface

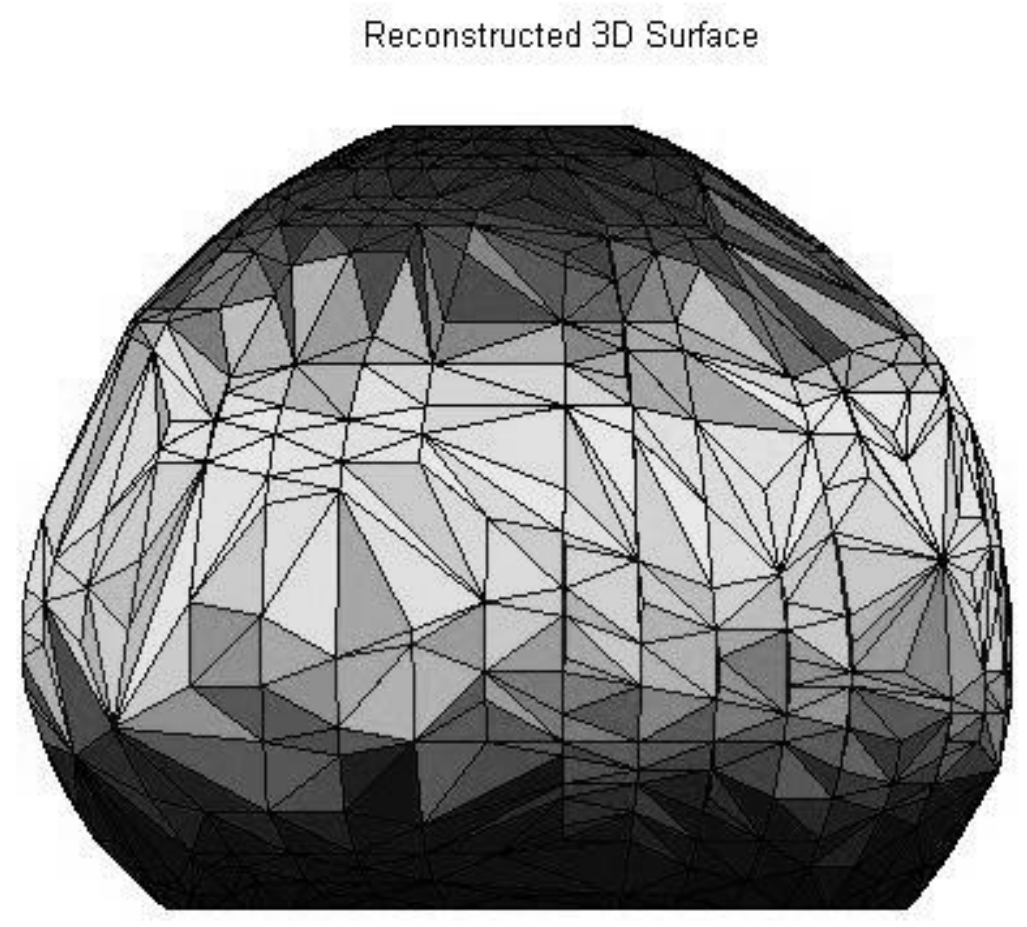

Figure 40: 2D Plot of 3D Reconstructed Point Cloud of Phantom 3 

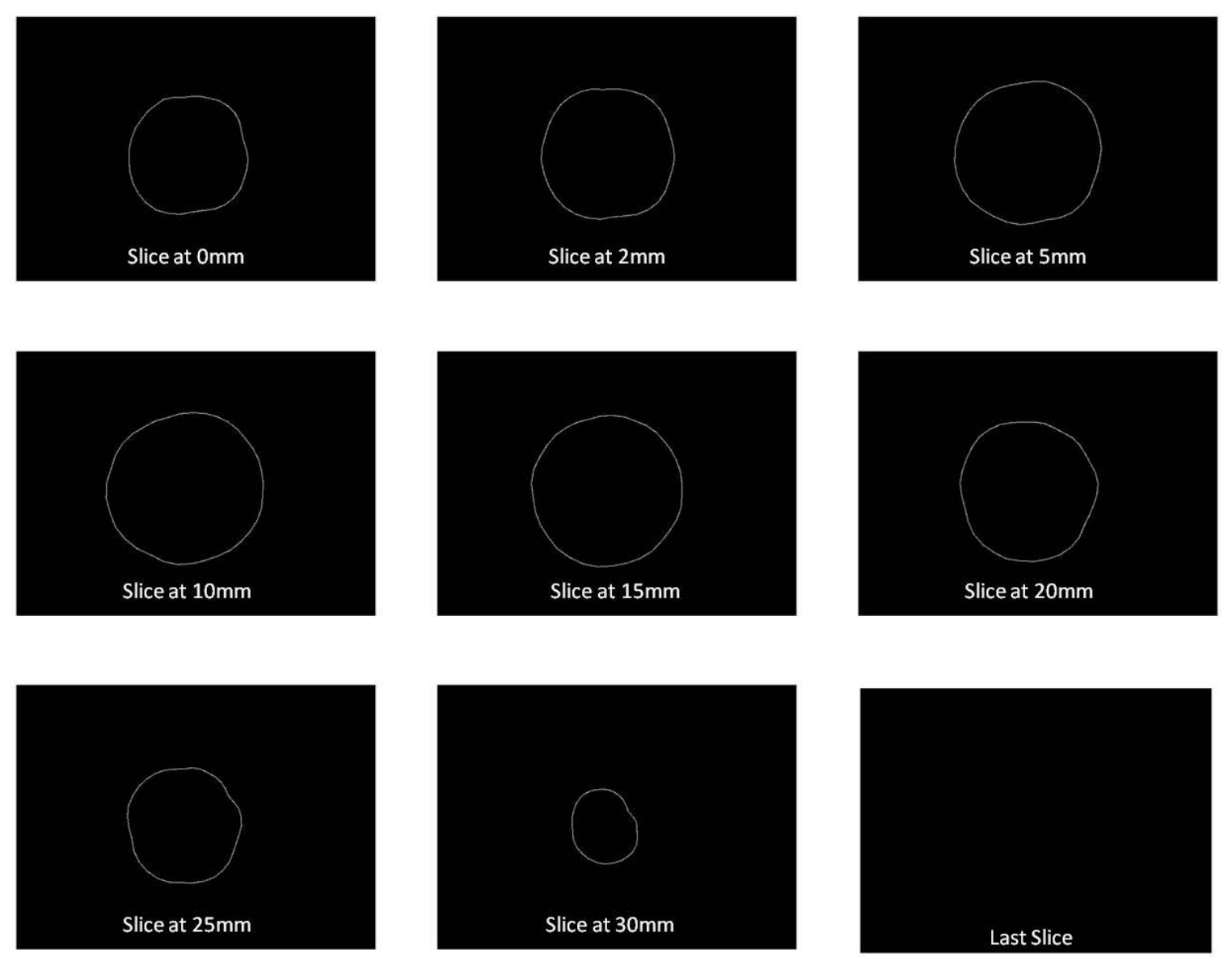

39

Figure 41: Slice of Phantom 3 with Irregular Surface shown in

Figure

\subsection{Image Fusion}

\subsubsection{Image Fusion with Phantom}

Figure 42 shows the phantom used for image fusion. Three radioactive beads of 5 $\mathrm{mm}$ diameter were taken and placed in the phantom. Radioactive beads are visible to the PET cameras while the physical outline of the phantom is visible to the optical camera. The reconstructed slices from both the systems are merged and the result is as shown in Figure 43. Slices are generated from $Y=Y_{0}$ to $Y=1$ and the last slice is the end of the image space where $\mathrm{Y}=1$. 


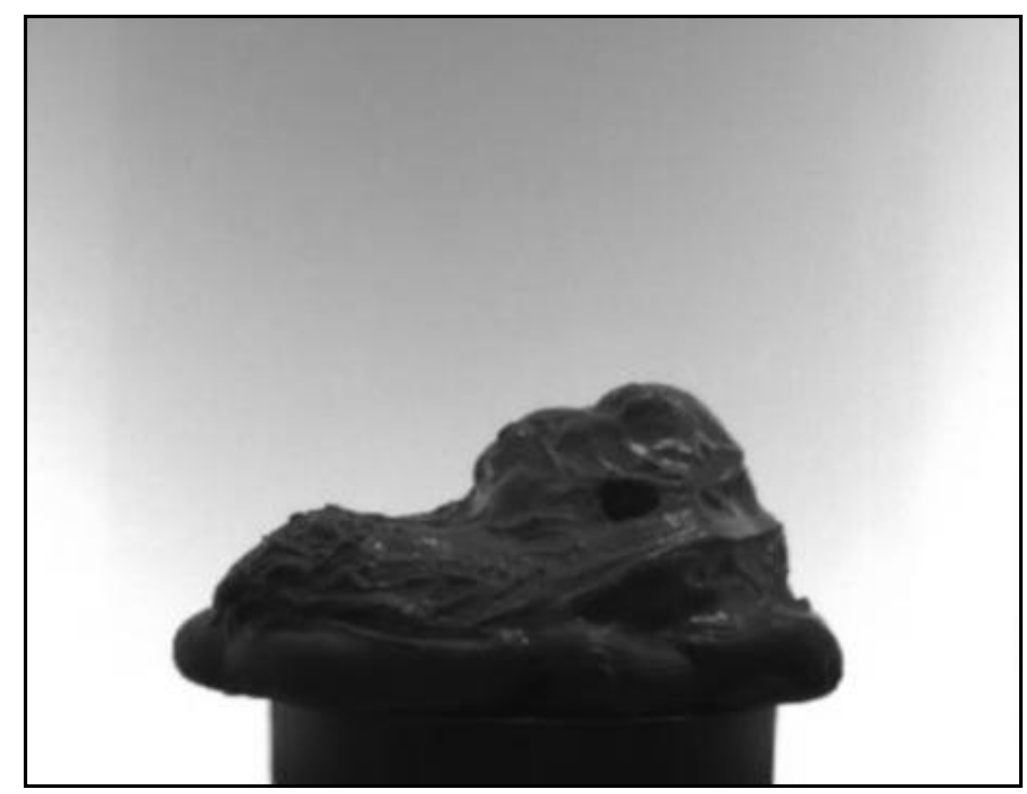

Figure 42: Phantom used for Image Fusion
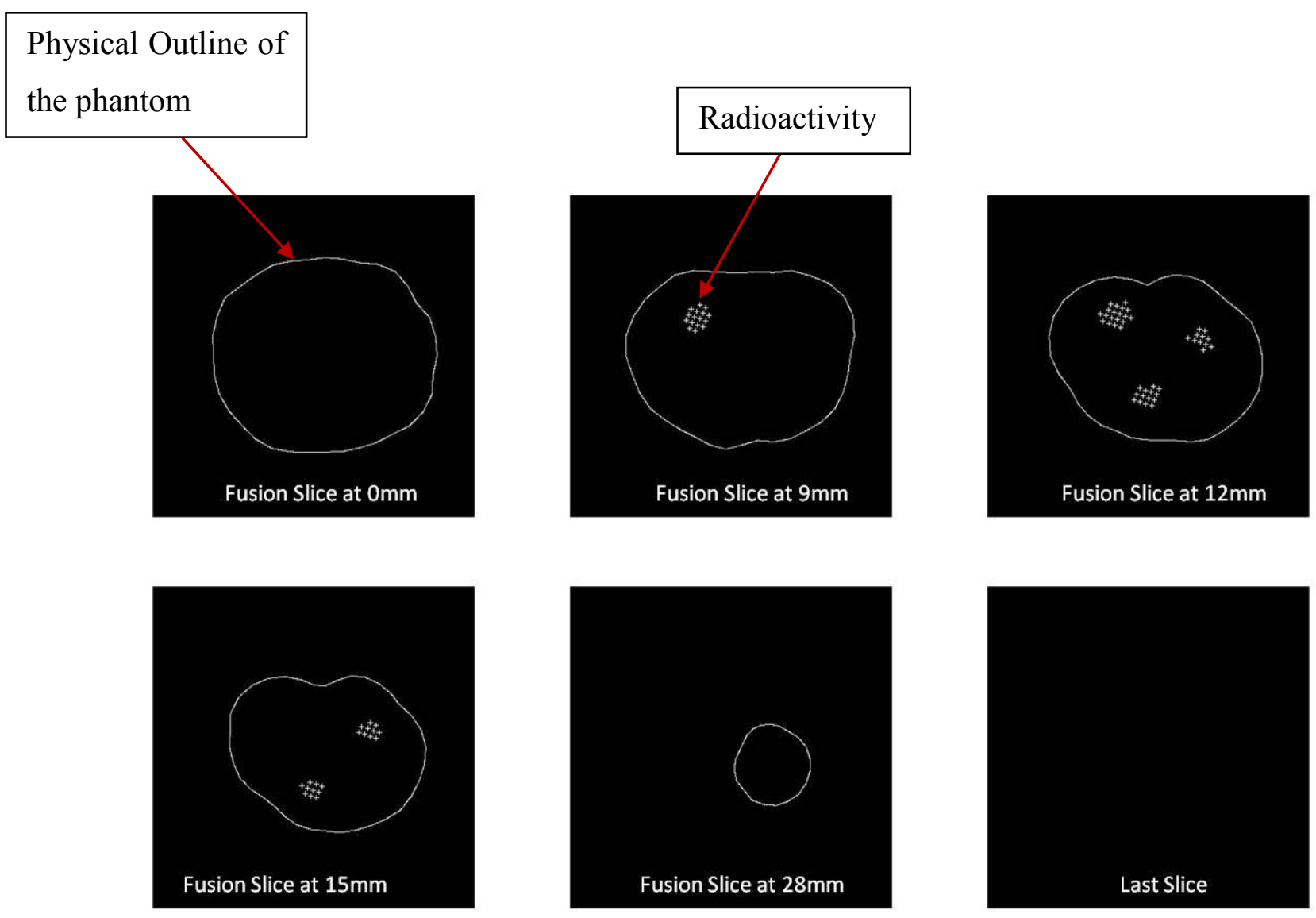

Figure 43: Slices of the Fusion Phantom 
In Figure 43 slice at $0 \mathrm{~mm}$ has no activity present so only the physical outline of the phantom is shown. In the other slices at $9 \mathrm{~mm}, 12 \mathrm{~mm}$ and $15 \mathrm{~mm}$ activity is present and it is shown by the small patches inside the physical outline. The slice at $29 \mathrm{~mm}$ has no activity so only the physical outline of the phantom is present. The last slice is the top of the image where the object is not present so it is a blank image.

\subsubsection{Margin Evaluation}

After the slices are merged, the PET points are identified in the superior, medial, inferior and lateral direction. In the same directions optical points are also identified. The distance between the corresponding points is calculated and this distance is the margin. If the margin is greater than $5 \mathrm{~mm}$ it is considered a negative margin and if the margin is less than $5 \mathrm{~mm}$ it is considered to be a positive margin. Figure 44 shows two slices whose margins are evaluated. The margins in the four directions are represented by four different colors. The superior is represented by red arrow, medial by orange, inferior by blue and lateral by orange respectively. The slice at $6 \mathrm{~mm}$ is found to have negative margins in the superior, medial, inferior and lateral directions with the margins being:

- Superior: Negative Margin. The margin is $10 \mathrm{~mm}$

- Inferior: Negative Margin. The margin is $32 \mathrm{~mm}$

- Medial: Negative Margin. The margin is $39 \mathrm{~mm}$

- Lateral: Negative Margin. The margin is $16 \mathrm{~mm}$

The slice at $19 \mathrm{~mm}$ is found to have positive margins in the inferior and lateral directions while it has negative margins in superior and medial directions with the margins being:

- Superior: Negative Margin. The margin is $10 \mathrm{~mm}$

- Inferior: Positive margin. The margin is $4 \mathrm{~mm}$

- Medial: Negative Margin. The margin is $23 \mathrm{~mm}$

- Lateral: Positive Margins. The margin is $3 \mathrm{~mm}$ 

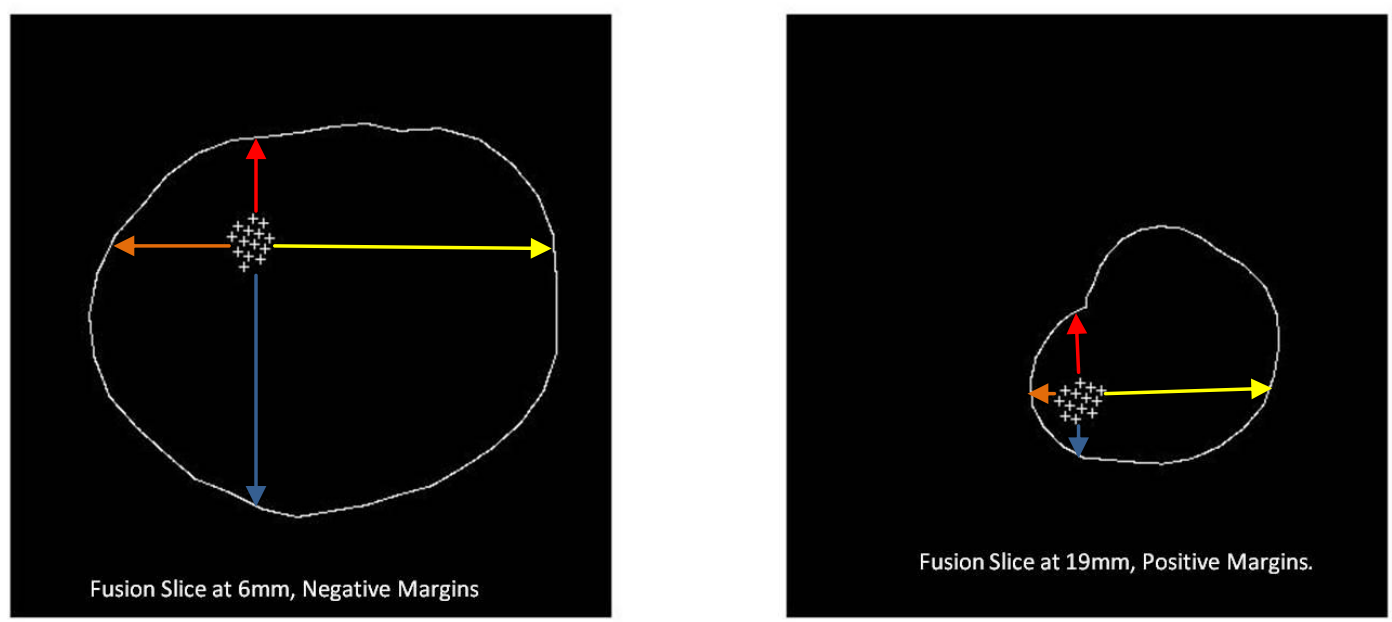

Figure 44: Margin Assessment of the Slices

\subsection{Error Analysis}

\subsubsection{Intervals in the degree of rotation}

A squash ball was taken as the test phantom. Readings were taken by rotating the phantom from 0 to 350 degrees at different intervals. Error analysis was performed for different intervals and the 10 degree interval was selected based on a tradeoff between the total time which is the sum of computation time and data acquisition time and the percentage error. The interval within the 360 degree of rotation was accepted as 10 degree based on the values in

The percentage error is calculated as shown in the Figure 45: 


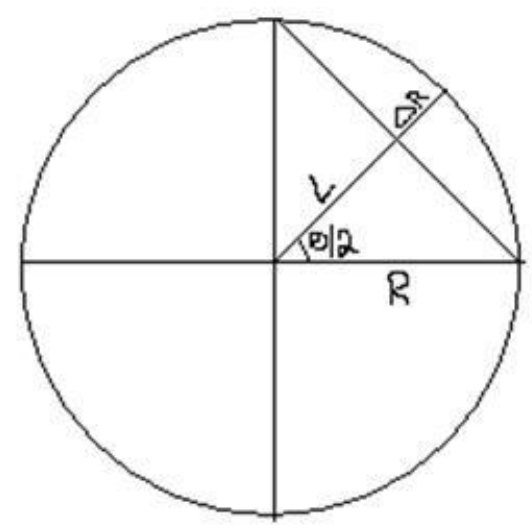

Figure 45: Error analysis based on degree of rotation

Let

$$
\begin{aligned}
& \theta=\text { Angle of rotation } \\
& R=\text { Radius of the circle } \\
& \Delta R=R-L \\
& \Delta R=R\left(1-\cos \left(\frac{\theta}{2}\right)\right) \\
& \text { PercentageError }=(\Delta R / R) * 100
\end{aligned}
$$

Table 5. It is observed that percentage error increases from $0.004 \%$ to $0.381 \%$ from 1 degree to 10 degrees while the total time is vice versa. The permissible error limit was considered to be less than or equal to $0.5 \mathrm{~mm}$. All the intervals of DOR are found to be within the permissible error limits. Figure 46 shows the tradeoff between the total time and percentage error to be between 4 or 5 degrees. The percentage error for 10 degrees of rotation is $0.308 \%$ which is still within the acceptable error limits. As the error is within the acceptable limits and the total time is also within the required time frame of 20 minutes for the whole processing of the dual modality PET/Optical imager.

The percentage error is calculated as shown in the Figure 45: 


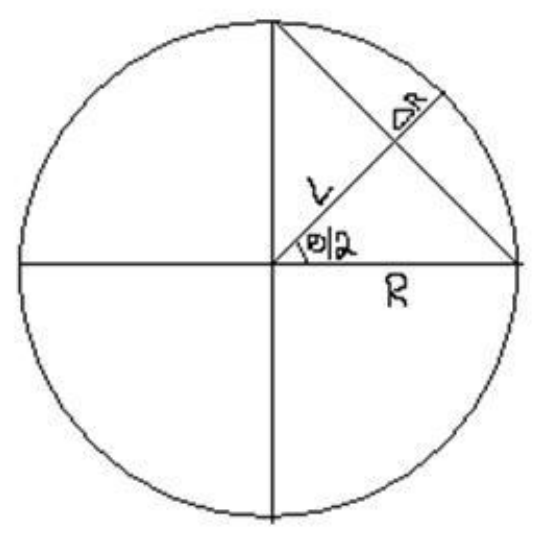

Figure 45: Error analysis based on degree of rotation

Let

$$
\begin{aligned}
& \theta=\text { Angle of rotation } \\
& R=\text { Radius of the circle } \\
& \Delta R=R-L \\
& \Delta R=R\left(1-\cos \left(\frac{\theta}{2}\right)\right) \\
& \text { PercentageError }=(\Delta R / R) * 100
\end{aligned}
$$

Table 5: Error Analysis for the intervals of Degree of Rotation

\begin{tabular}{|l|l|l|l|l|}
\hline $\begin{array}{l}\text { Degree of } \\
\text { Rotation } \\
\text { (Degrees) }\end{array}$ & $\begin{array}{l}\text { Computation } \\
\text { Time (min:sec) }\end{array}$ & $\begin{array}{l}\text { Data Acquisition } \\
\text { Time (min:sec) }\end{array}$ & $\begin{array}{l}\text { Total Time } \\
(\mathbf{m i n}: \mathbf{s e c})\end{array}$ & $\begin{array}{l}\text { Percentage } \\
\text { Error } \\
(\Delta \mathbf{R} / \mathbf{R}) * 100\end{array}$ \\
\hline 1 & $3: 89$ & $4: 42$ & $8: 31$ & 0.004 \\
\hline 2 & $1: 97$ & $2: 45$ & $4: 42$ & 0.015 \\
\hline 3 & $1: 41$ & $2: 05$ & $3: 46$ & 0.034 \\
\hline 4 & $1: 11$ & $1: 42$ & $2: 53$ & 0.061 \\
\hline 5 & $1: 02$ & $1: 32$ & $2: 34$ & 0.095 \\
\hline 6 & $0: 49$ & $1: 24$ & $1: 73$ & 0.137 \\
\hline 8 & $0: 39$ & $1: 15$ & $1: 54$ & 0.244 \\
\hline 9 & $0: 39$ & $1: 12$ & $1: 51$ & 0.308 \\
\hline 10 & $0: 34$ & $1: 10$ & $1: 44$ & 0.381 \\
\hline
\end{tabular}




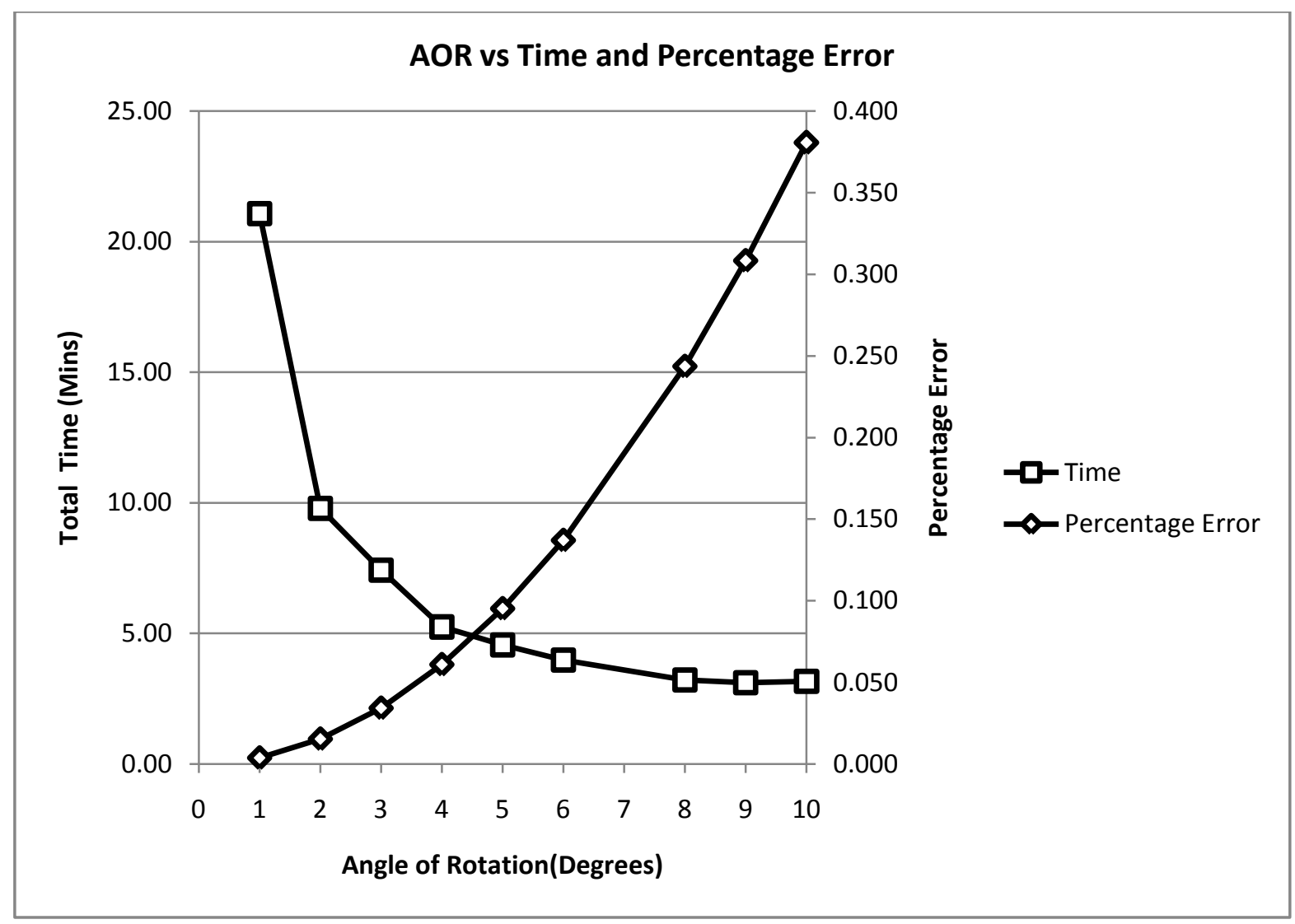

Figure 46: Angle of Rotation versus Total time and Percentage Error 


\section{Conclusions and Future Study}

\subsection{Conclusion from the Experimental Results}

Margin assessment procedures play an important role in determining the success rate of lumpectomy breast cancer surgeries. This has led to the development of a dual modality margin specification imager. The imager was tested with the PET and optical modalities to assess the margins.

Optical modality has been designed and developed for the DMI. It has been shown that the $3 \mathrm{D}$ reconstruction can be done for a squash ball with an accuracy of $\pm 1 \mathrm{~mm}$ to the original diameter in $\mathrm{X}, \mathrm{Y}$ and $\mathrm{Z}$ directions respectively. The $3 \mathrm{D}$ reconstruction of the surface of a digital phantom was obtained within the range of \pm 1 pixel to the original diameter in $\mathrm{X}$ and $\mathrm{Y}$ directions. The algorithm works for various phantoms with irregular surfaces and also works well for different sizes of phantoms. The basic idea that has been implemented to obtain 3D volumes from both the modalities and to evaluate the margins on the merged volumes was successful. The excised breast tissue was assumed to be convex in shape, based on discussions with the surgeon. The current algorithm implemented might not give the desired results in recontructing phantoms with sharp corners and concave shaped structures. It was not possible to test with real excised breast tissue due to various concerns.

The surgeon places stitches on the excised specimen which indicate the superior and lateral directions and help in orientation to relate back to the patient. Identification of the stitches is beyond the scope of current work. An alternative method was suggested to keep the orientation intact by using a specimen holder which has 12 o'clock, 3 o'clock, 6 o' clock and 9 o' clock markings. The excised breast tissue should be placed with care on the holder such that superior lies at 12 o' clock and lateral at 9 o' clock positions respectively. Also the superior, lateral, medial and inferior directions of the excised breast tissue can be colored with different dyes alternatively.

The successful design and development of the prototype of the dual modality PET/optical imager proves that this concept is potential enough for clinical trials with a robust system. 


\subsection{Future Study}

3D surface reconstruction of the excised breast tissue has the potential for further research. Structured light can be utilized to perform 3D reconstruction of the excised breast tissue and it is potential enough to provide high end resolution and improve the accuracy levels. User-friendly interface can be developed for easy use by the surgeon. The speed and accuracy of the algorithm can be further improved.

If the tests can be performed with real breast tissue the real time challenges of the optical modality can be identified and handled which would be helpful in obtaining the desired results during future clinical trials. 


\section{Bibliography}

1. Long-term results of a randomized trial comparing breast conserving therapy with astectomy: European Organization for Research and Treatment of Cancer 10801 trial. van Dongen . J, Voogd A.C., et al. 14, July 2000, Journal of the National Cancer Institute, Vol. 92, pp. 1143-1150.

2. Second Malignancies after treatment of early-stage breast cancer: lumpectomy and radiation therapy versus mastectomy. Obedian E, Fischer DB, HafftyBG. 12, June 2000, Journal of Clinical Oncology, Vol. 18, pp. 2406-2412.

3. Breast Cancer. Sharma M, Abraham J. 2001, The Bethesoa Handbook of Clinical Oncology, pp. 151-173.

4. Positive margins: the challenge continues for breast surgeons. L, Jacobs. 5, May 2008, Annals of Surgical Oncology, Vol. 15 , pp. 1271-1272.

5. PET Imaging in Breast Cancer. Bombardierie. E, Crippa. F. 3, 2001, The Quarterly Journal of Nuclear Medicine, Vol. 45, pp. 245-256.

6. Minimizing local recurrence after breast conserving therapy using intraoperative shaved margins to determine pathologic tumor clearance. Camp ER, McAuliffe PF, Gilroy JS, et al. 6, December 2005, Journal of the American College of Surgeons, Vol. 201 .

7. Influence of breast cancer margin assessment method on the rates of positive margins and residual carcinoma. Mendez JE, Lamorte WW, et al. 4, October 2006, The American Journal of Surgery, Vol. 192, pp. 538-540.

8. Individual and Combined Effects of Age, Breast Density, and Hormone Replacement Therapy Use on the Accuracy of Screening Mammography. Carney PA, Miglioretti DL, Yankaskas BC et al. 3, February 2003, Annals of Internal Medicine, Vol. 138 , pp. $168-175$. 
9. Breast Density as a Predictor of Mammographic Detection: Comparison of Interval- and Screen-Detected Cancers. Mendelson MT, Oestreicher N, Porter PL et al. 13, July 2000, Journal of the National Cancer Institute, Vol. 92, pp. 1081-1087.

10. Ultrasound for breast cancer screening and staging. Gordon, Paula B. 3, May 2002, Radiologic Clinics of North America, Vol. 40, pp. 431-441.

11. New Diagnostic Techniques for Breast Cancer Detection. Singh V, Saunders C, Wylie L and Bourke A. 4, 2008, Future Oncology, Vol. 4, pp. 501-503.

12. American Cancer Society Guidelines for Breast Cancer Screening with MRI as an Adjunct to Mammography. Saslow D, Boets C, Burke W et al. 2, March-April 2007, A Cancer Journal for Clinicians, Vol. 57, pp. 75-89.

13. Beyond Standard Mammographic Screening: Mammography at Age Extremes, Ultrasound, and MR Imaging. Berg, Wendie A. 5, 2007, Radiologic Clinics of North America, Vol. 45, pp. 895-906.

14. Positron Emission Tomography (PET): an update on applications in breast cancer. Wahl, Richard L. 3-4, August 1998, Breast Disease, Vol. 10, pp. 165-175.

15. Diagnostic Value of Positron Emission Tomogrpahy for Detecting Breast Cancer. Noh D-Y, Yun I-J et al. 1998, World Journal of Surgery, Vol. 22, pp. 223-228.

16. Role of Positron-Emission Tomography Scan in the Diagnosis and Management of Breast Cancer. Almubarak M, Osman S, Marano G, Abraham J. 3, March 2009, Oncology, Vol. 23, pp. 255-261.

17. Breast Imaging With Positron Emission Tomography and Fluorine-18 Fluorodeoxyglucose: Use and Limitations. Avril .N, Rose . C. A et al. 20, October 2000, Journal of Clinical Oncology, Vol. 18, pp. 3495-3502.

18. The Chocie of the Correct Imaging Modality in Breast Cancer Management. Bombardieri . E, Gianni . L. Supplement 1, June 2004, European Journal of Nuclear Medicine and Molecular Imaging, Vol. 31, pp. S179-S186. 
19. Positron Emission Tomography and Breast Masses: Comparision with Clinical, Mammographic, and Pathological Findings. Crowe . P. J, Adler. P. L, Shenk .R. Robert and Sunshine . J. 2, March 1994, Annals of Surgical Oncology, Vol. 1, pp. 132-140.

20. Prospective multicenter study of axillary nodal staging by positron emission tomography in breast cancer: a report of the staging of breast cancer with PET study group. Wahl, R. and Siegel, B. A., et al. 2, June 2004, Women's Oncology Review, Vol. 4, pp. p161-162.

21. A comparative study on the value of FDG-PET and sentinel node biopsy to identify occult axillary metastases. Veronesi U, De Cicco C, Galimberti VE et al. 3, March 2007, Annals of Oncology, Vol. 18, pp. 473-478.

22. Determinants of Diagnostic Performance Of [F-18]Fluorodeoxyglucose Positron Emission Tomography for Axillary Staging in Breast Cancer. van der Hoeven JJ, Hoekstra OS, Comans EF, et al. No 5, 2002, Annals of Surgery, Vol. 236, pp. 619-624.

23. Assessment of Axillary Lymph Node Involvement in Breast Cancer Patients With Positron Emission Tomography Using Radiolabeled 2-(Fluorine-18)- fluoro-2-deoxy-Dglucose. Avril n, Dose J, Janicke F, et al. No 17, 1996, Journal of the National Cancer Institute, Vol. 88, pp. 1204-1209.

24. Preoperative FDG-PET for Axillary Metastases in Patients With Breast Cancer. Chung A, Liou D, Karlan S, et al. No . 8, August 2006, Archives of Surgery, Vol. 141, pp. 783-789.

25. Staging of the Axilla in Breast Cancer Accurate In Vivo Assessment Using Positron Emission Tomography With 2-(Fluorine-1 8)-Fluoro-2-Deoxy-D-Glucose. Smith IC, Ogston KN, Whitford P, et al. No 2, 1998, Annals of Surgery, Vol. 228, pp. 220-227.

26. Positron emission tomography scanning in gynecologic and breast cancers. Zimmy M, Siggelkow W. 1, Feb 2003, Current opinion in obstetrics and gynecology, Vol. 15, pp. 69-75. 
27. F-18 Flourodeoxy glucose positron emission tomography in the diagnosis of tumor recurrence and metastases in the follow-up of patients with breast carcinoma: A comparision to conventional imaging. Gallowitsch HJ, Kresnik E, et al. 5, May 2003, Investigative Radiology, Vol. 38, pp. 250-256.

28. Impact of FDG-PET on defining the extent of disease and on the treatment of patients with recurrent or metastatic breast cancer. Eubank WB, Mankoff D, Battacharya M et al. 2, 2004, Vol. 183, pp. 479-486.

29. Performance of 2-deoxy-2-[F-18]flouro-D-glucose positron emission tomography and integrated PET/CT in restaged breast cancer patients, Molecular Imaging and Biology,. Feuger BJ, Weber WA, Quon A, et al. 5, Sep-Oct 2005, Vol. 7, pp. 369-376.

30. The value of FDG positron emission tomography in the management of patients with breast cancer. Weir L, Worsley D, Bernstein V. 3, 2005, The Breast Journal, Vol. 11, pp. 204-209.

31. Value of flourodeoxyglucose positron emission tomography in women with breast cancer. Landheer ML, Steffens M. G, et al. 2005, British Journal of Surgery, Vol. 92, pp. 1363-1367.

32. An intraoperative $3 D$ ultrasound system for tumor margin determination in breast cancer surgery. DeJean P, Brackstone M, Fenster A. 2, Feb 2010, Medical Physics, Vol. 37, pp. 564-570.

33. Attaining negative margins in breast-conservation operations: is there a consensus among breast surgeons? Blair SL, Thompson K, et al. 5, November 2009, Journal of the American College of Surgeons, Vol. 209, pp. 608-613.

34. A multi-site validation trial of radioactive seed localization as an alternative to wire localization. Hughes JH, Mason MC, et al. 2, Mar-Apr 2008, The Breast Journal, Vol. 14, pp. 153-157. 
35. Radioguided occult lesion localisation in breast cancer using an intraoperative portable gamma camera: first results. Paredes .P, et al. Number 2, February 2008, European Journal of Nuclear Medicine and Molecular Imaging, Vol. Volume 35.

36. Dual modality planar PET/optical scanner for imaging of surgical margins in extracted tissue samples. A. Stolin, C. Freeman, B. Kross, J. McKisson, J. Proffitt and S. Majewski. 2008. IEEE Nuclear Science Symposium Conference.

37. Niku, Saeed B. Introduction to Robotics Analysis, Systems, Applications.

38. Is Breast MRI EVer Useful in a Mammographic Screening Programme? Warren RM, Crawley A. 2002, Clinical Radiology, Vol. 57, pp. 1090-1097. 


\section{Appendix A}

\section{Source Codes}

\subsection{Pixel to millimeter calibration}

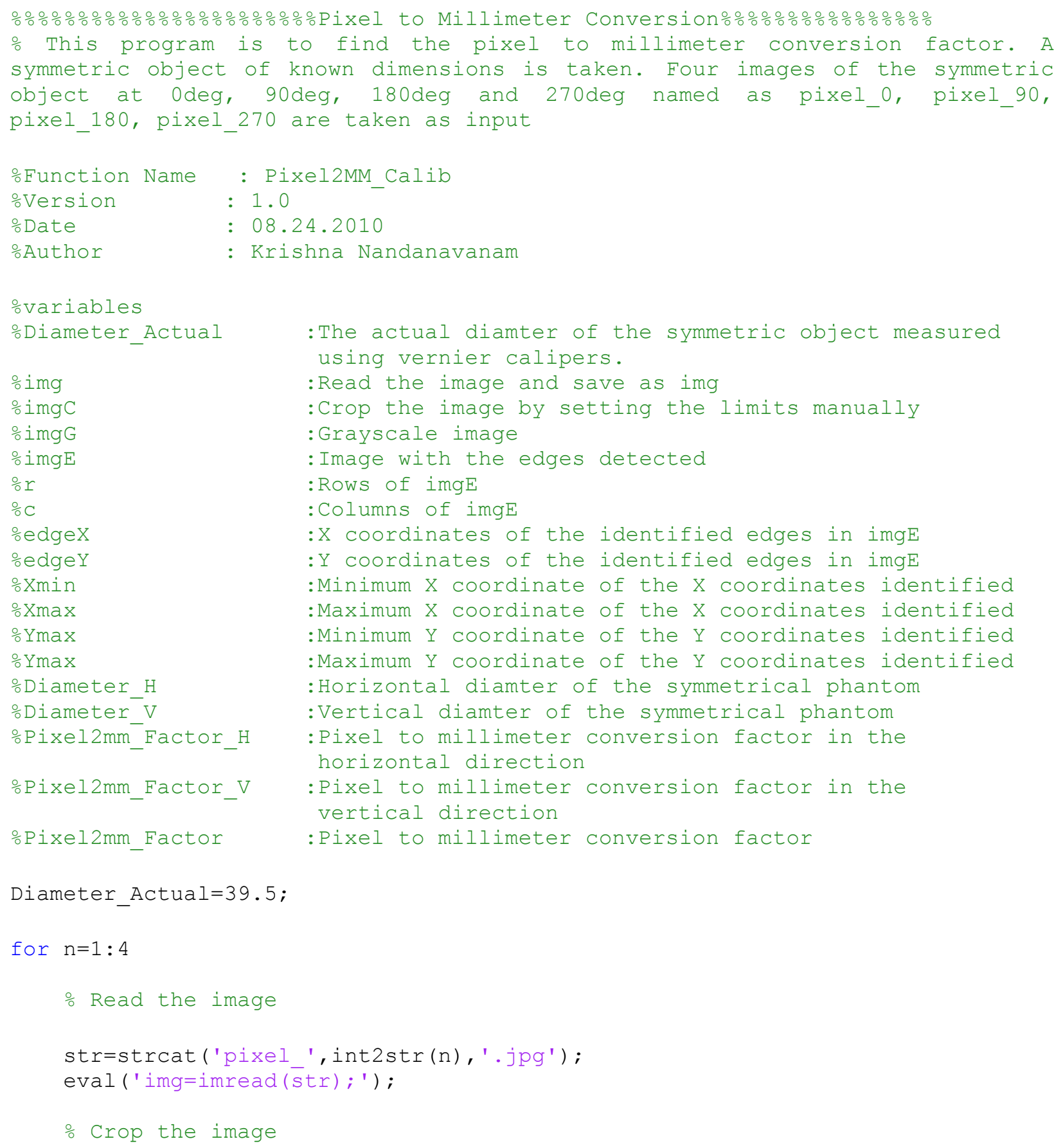




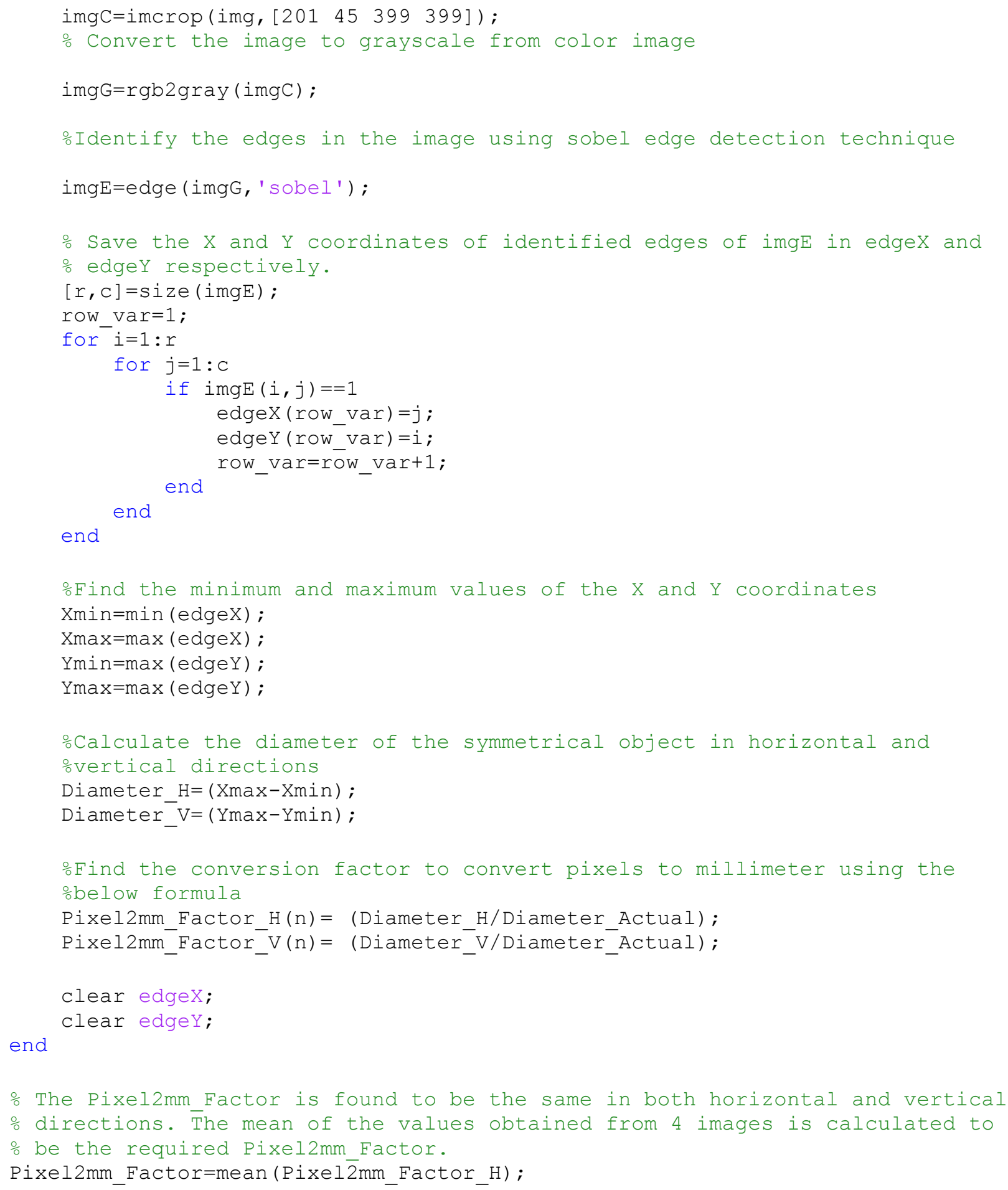




\subsection{AOR calibration}

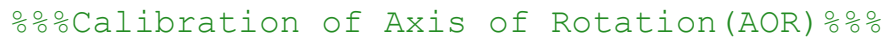

This program is to find $X 0$ and $Y O$, AOR in the image plane. 180 images of the capillary tube are taken into consideration where each image is taken at an angle of 2 degrees.

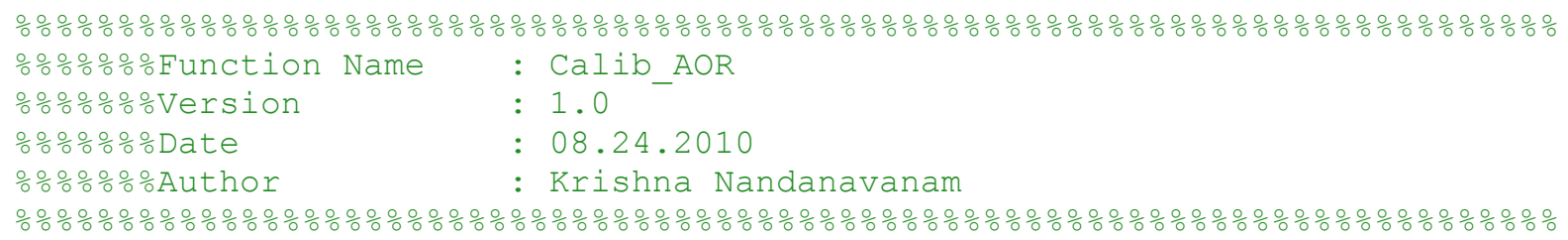

\% Read the image 


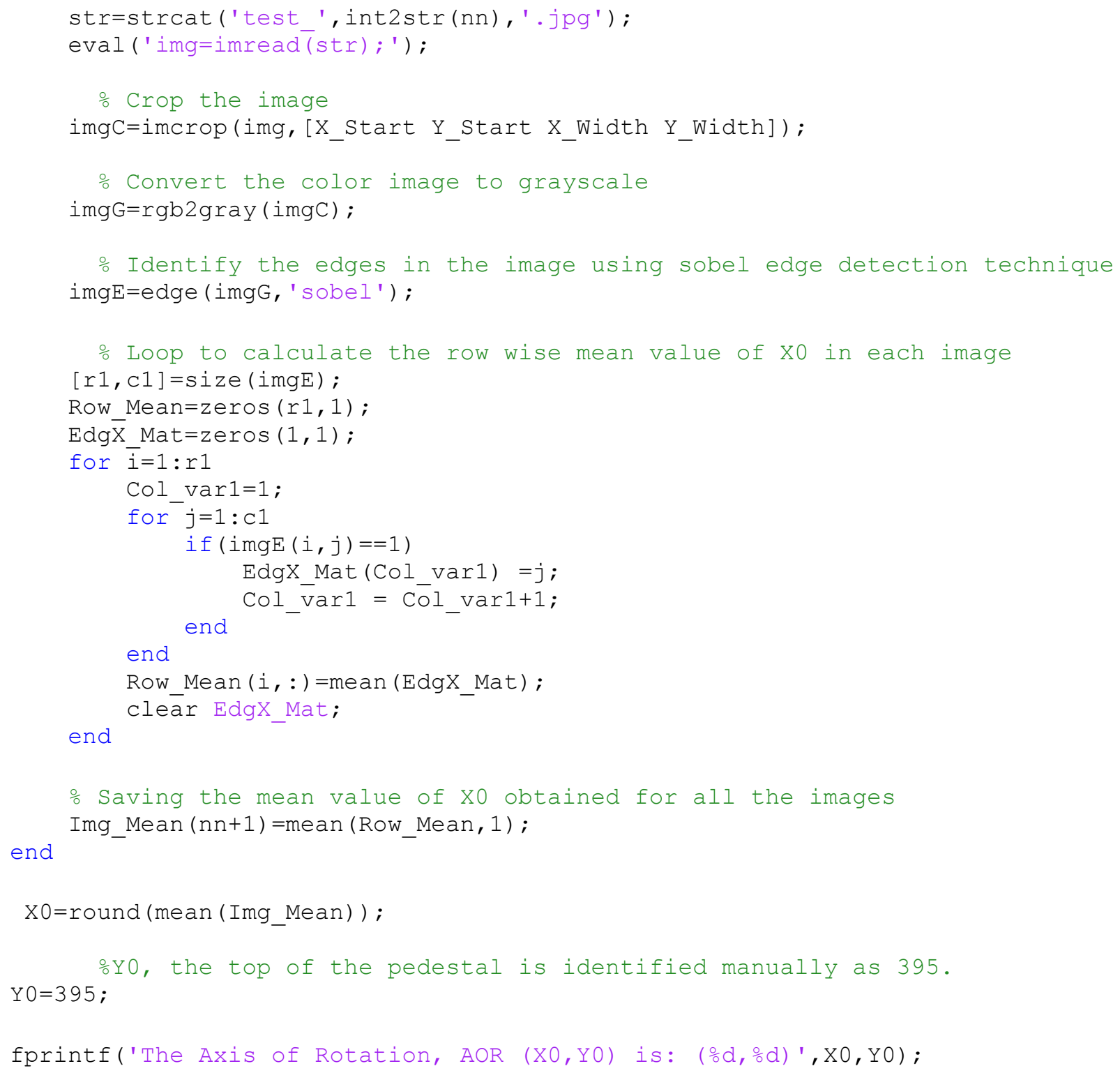




\subsection{Image Fusion Calibration}

\subsubsection{Identification of the centroids in optical modality}

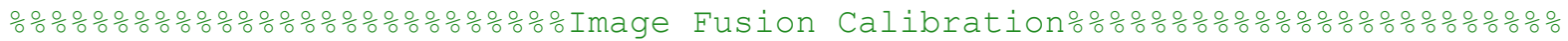
oThis program is to calculate the coordinates of the centroids of the four spheres in the calibration phantom in the optical system.

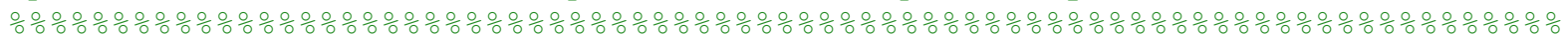

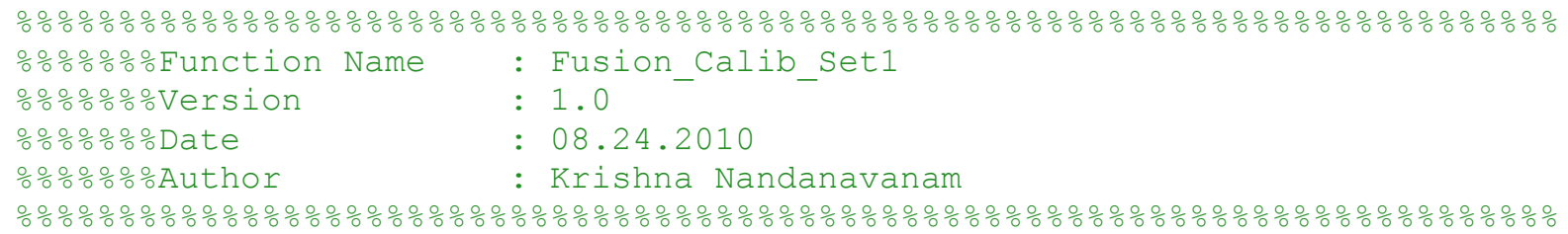




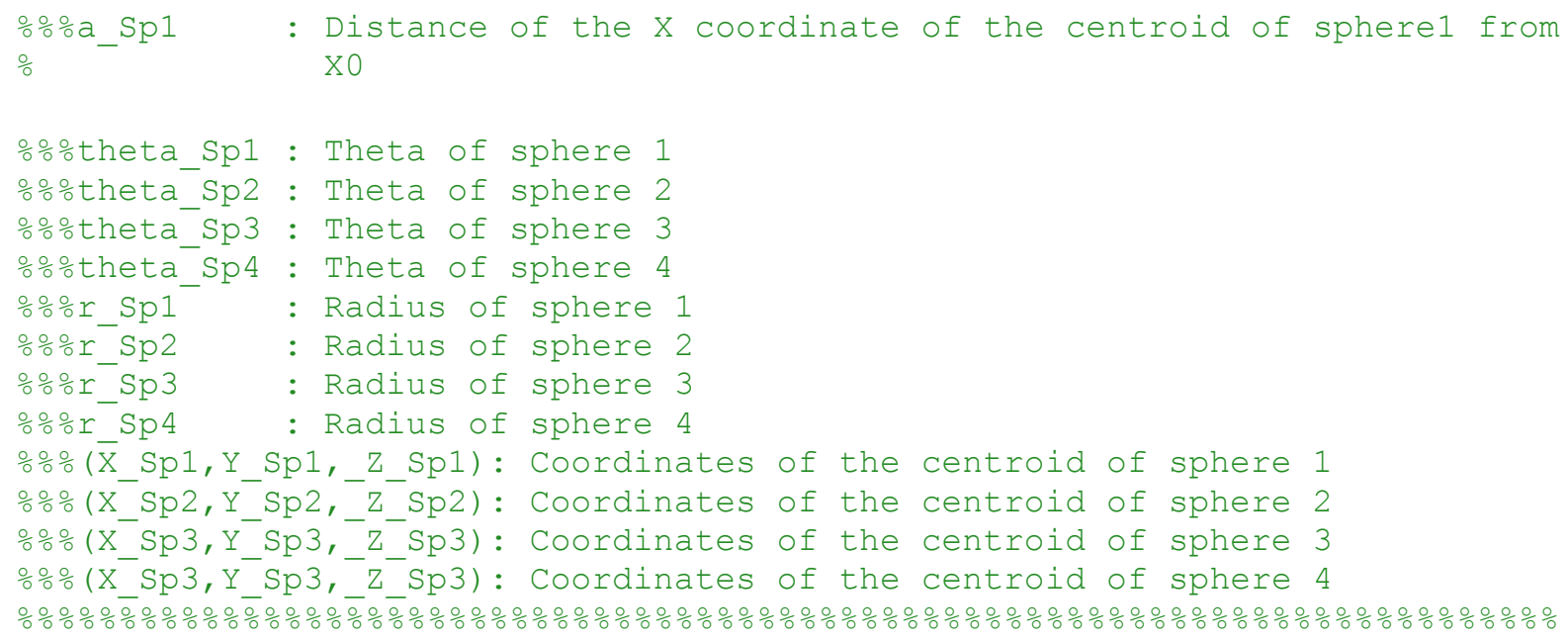




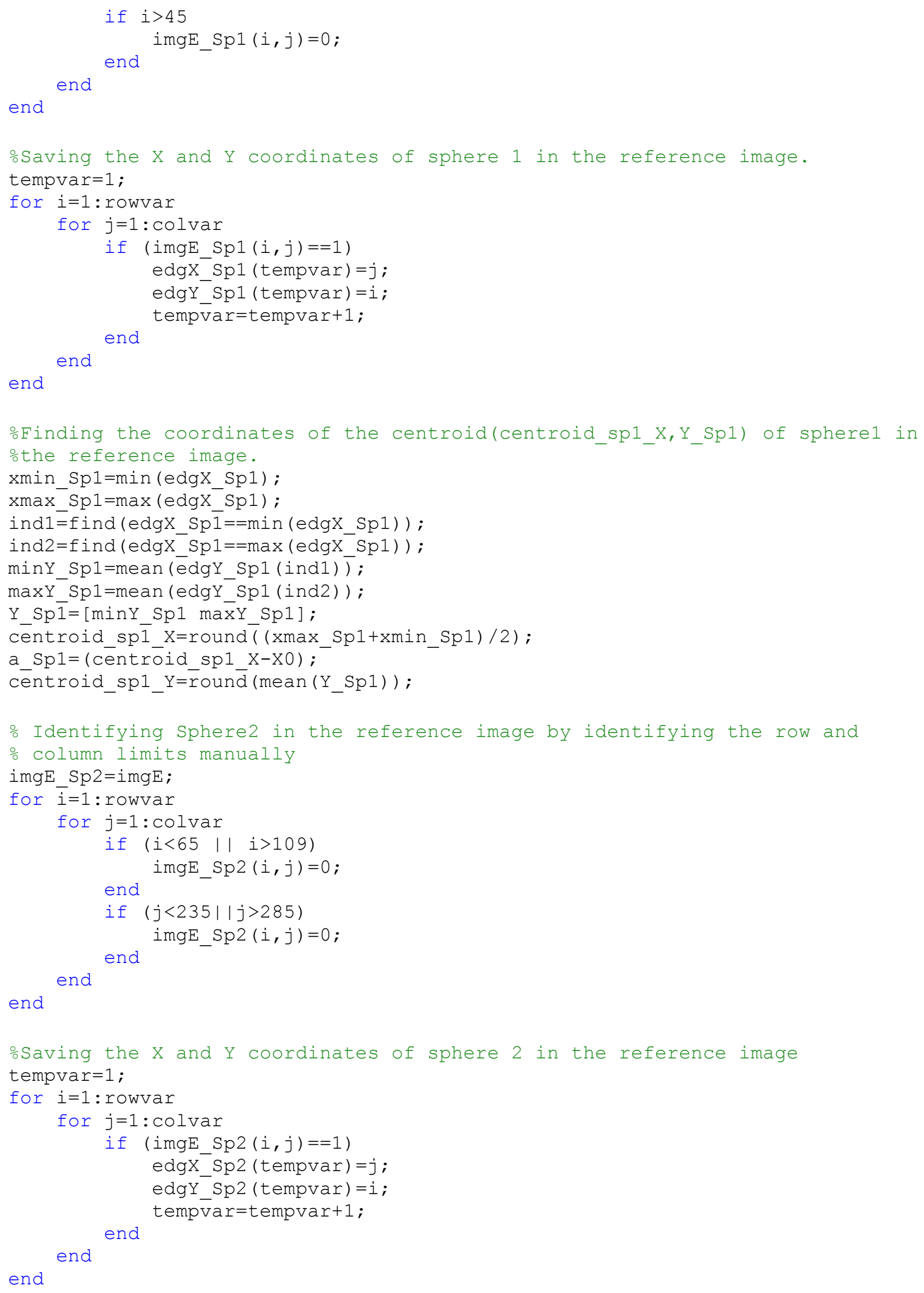




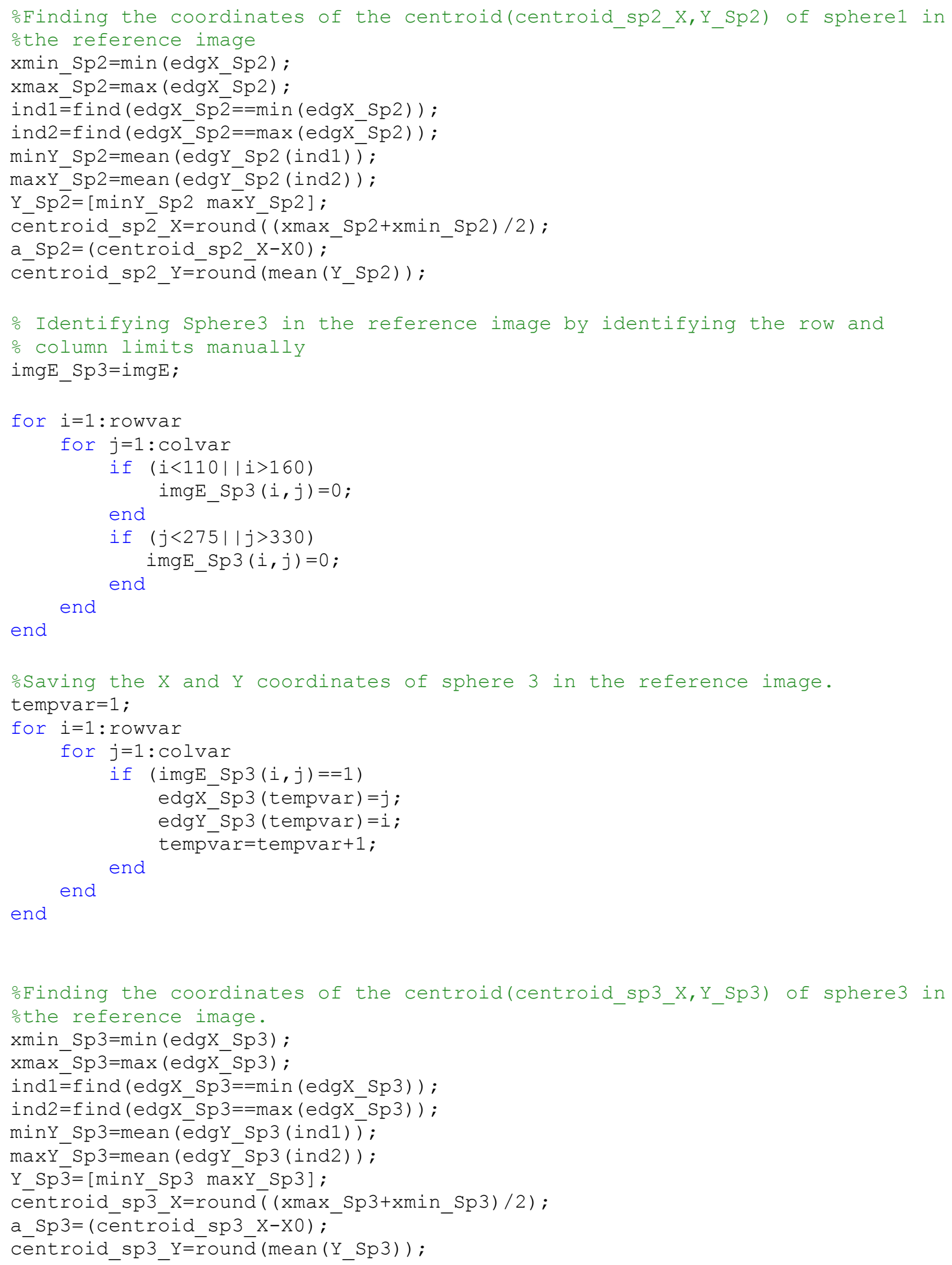









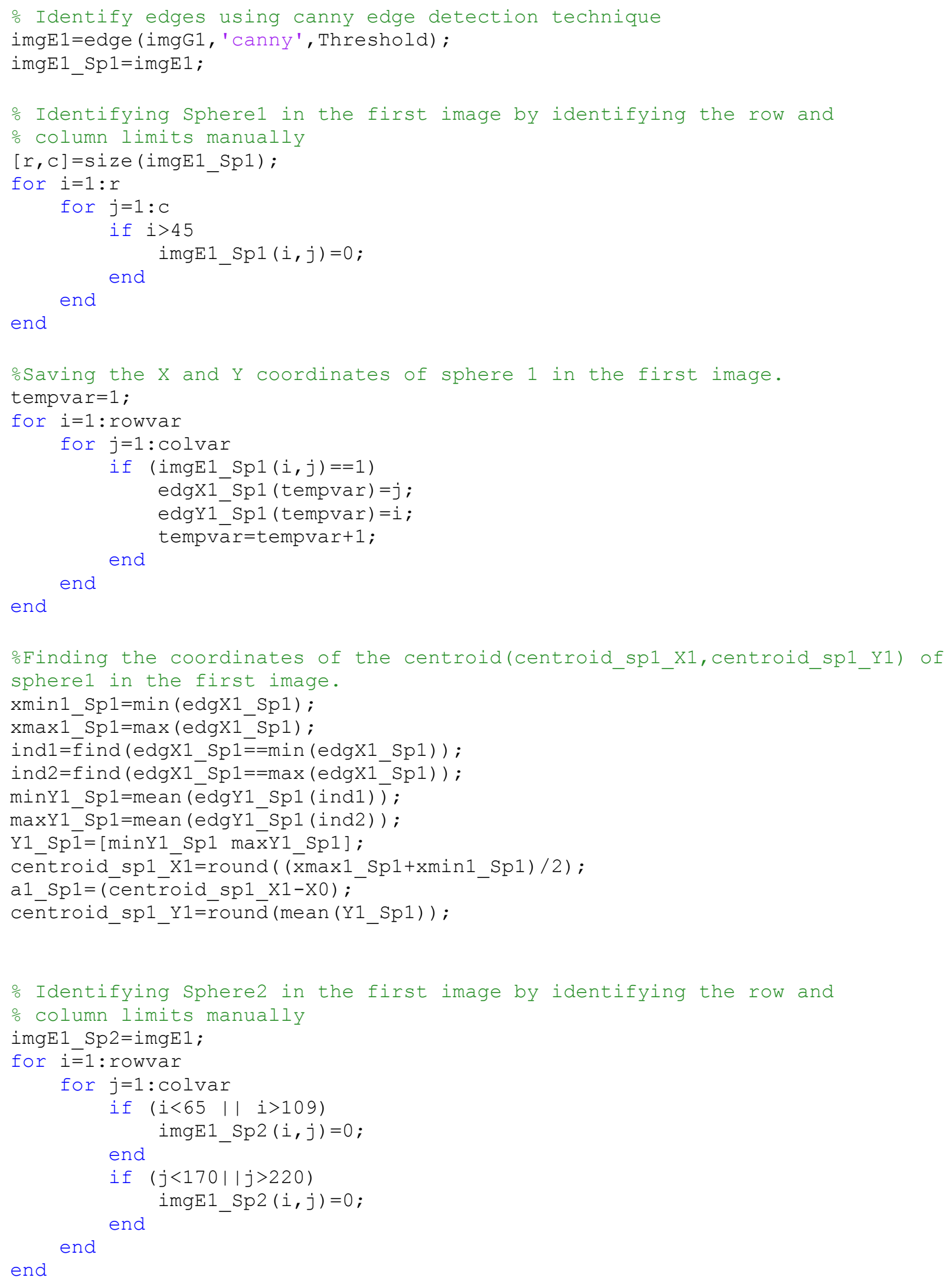




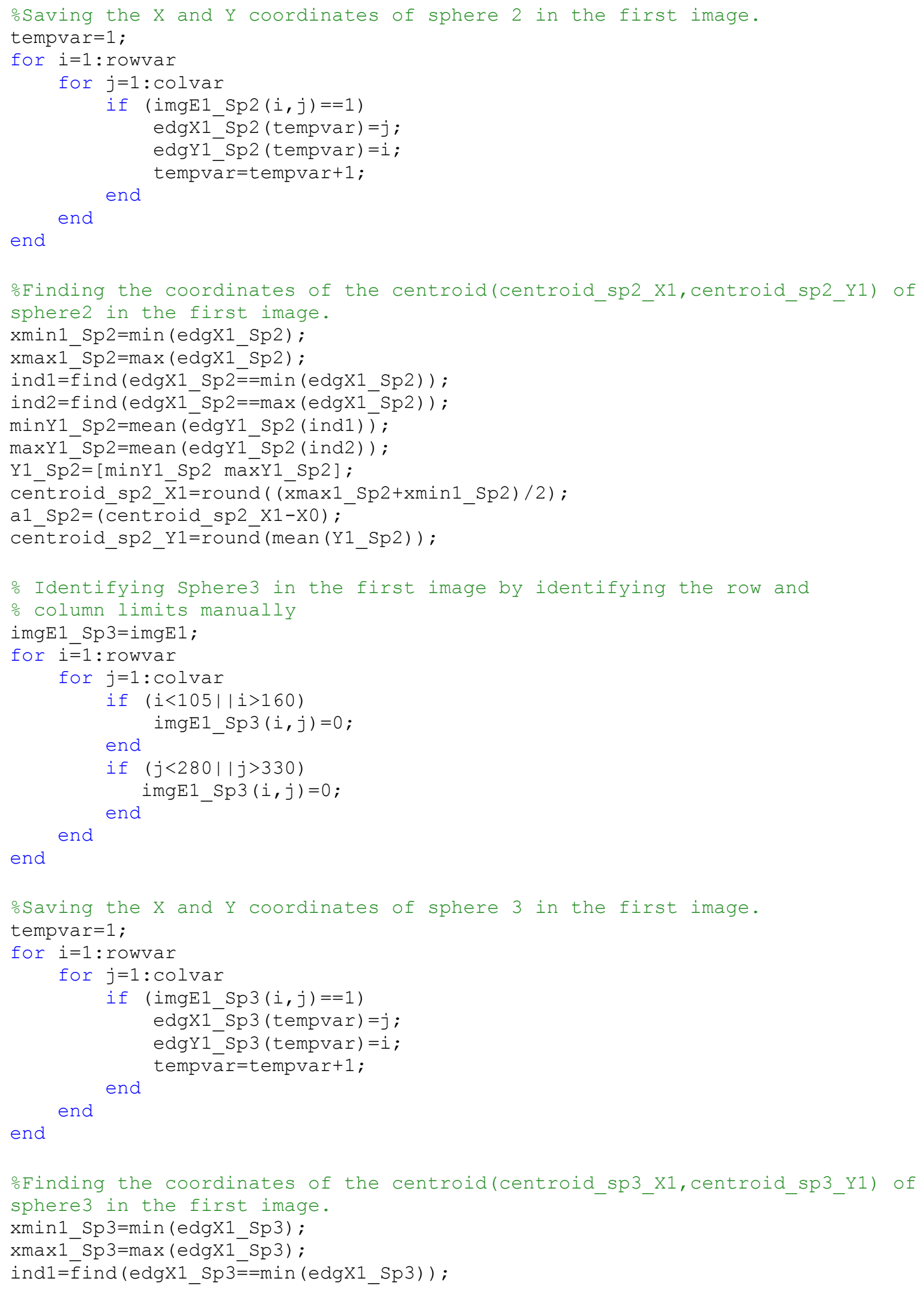




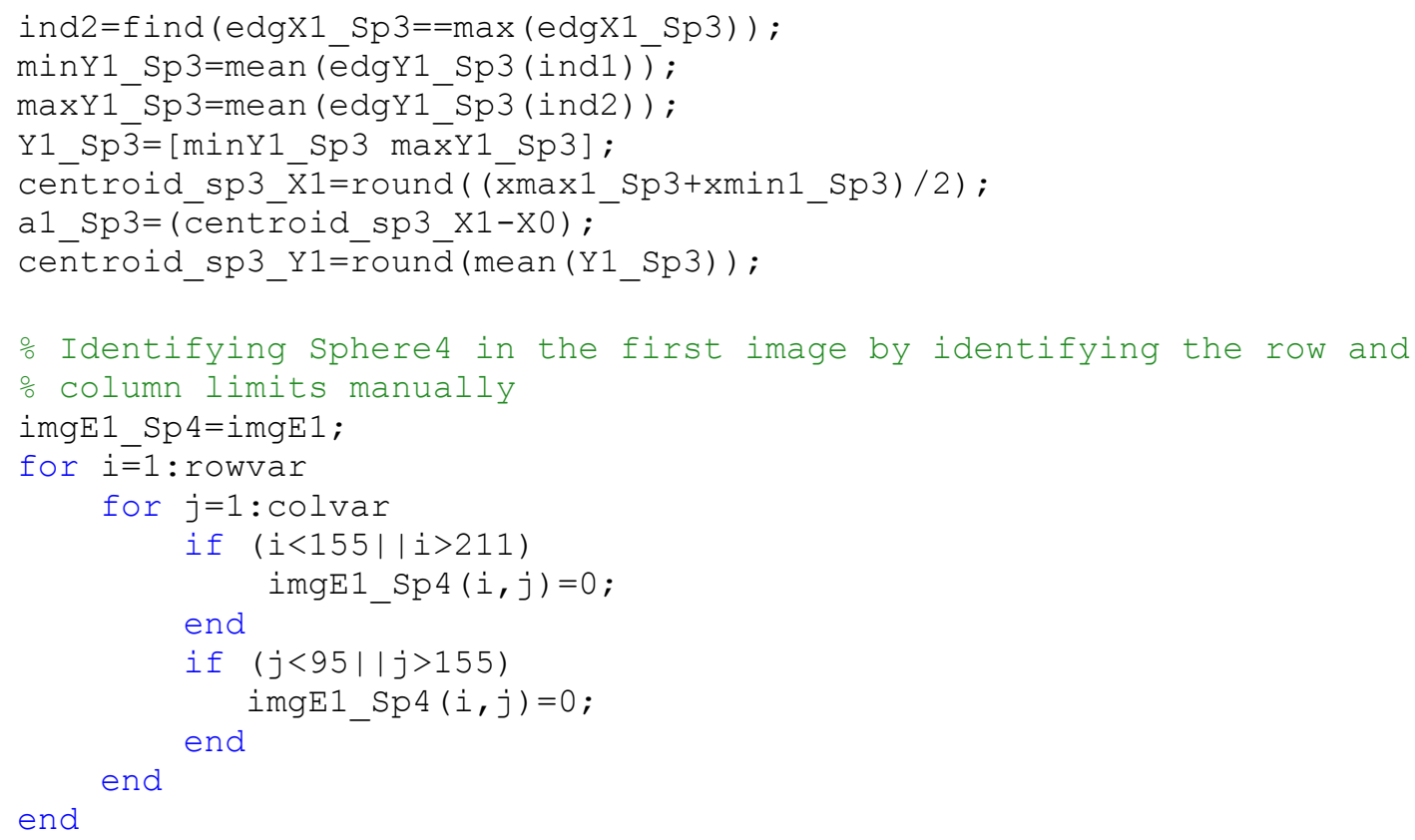




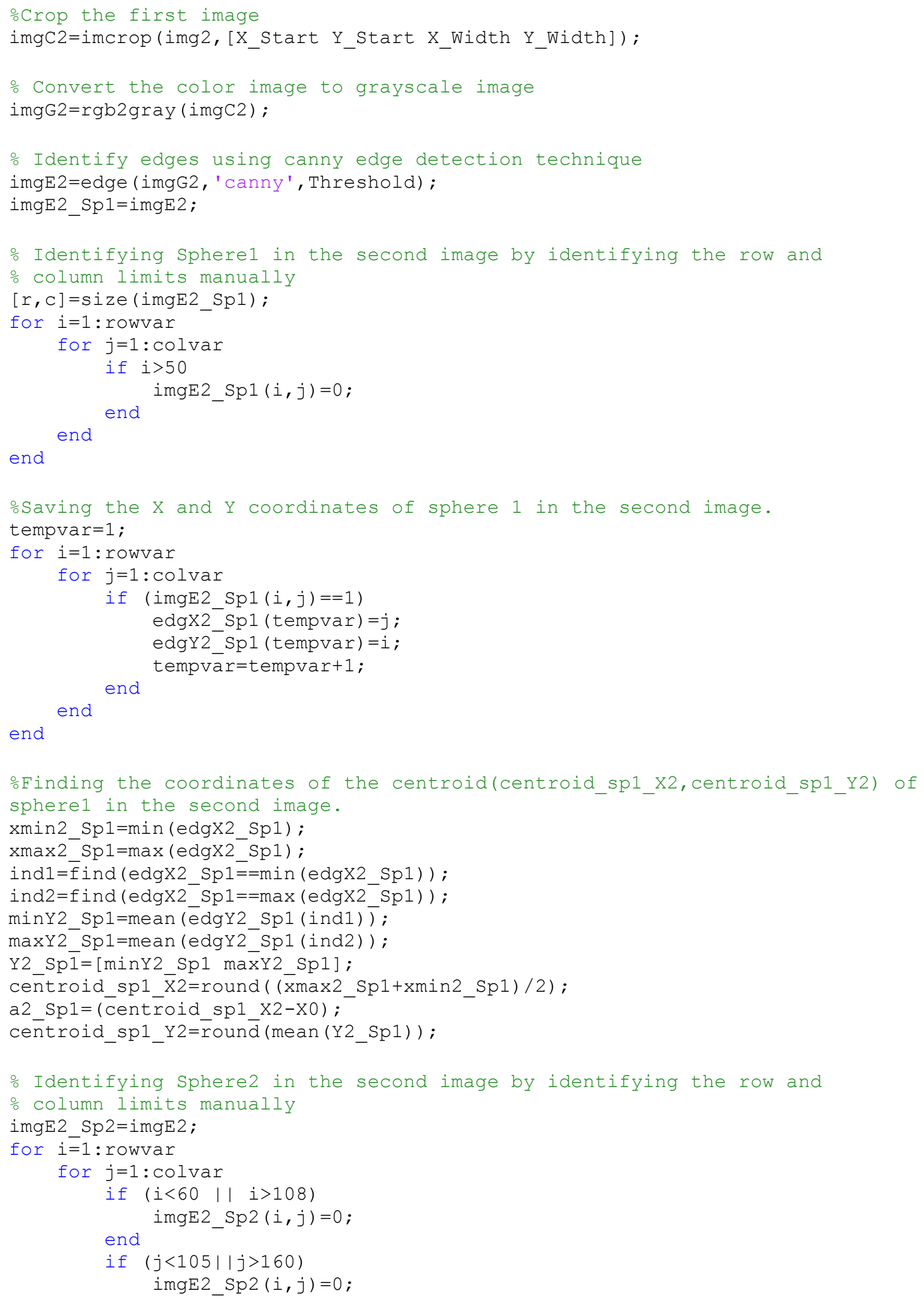




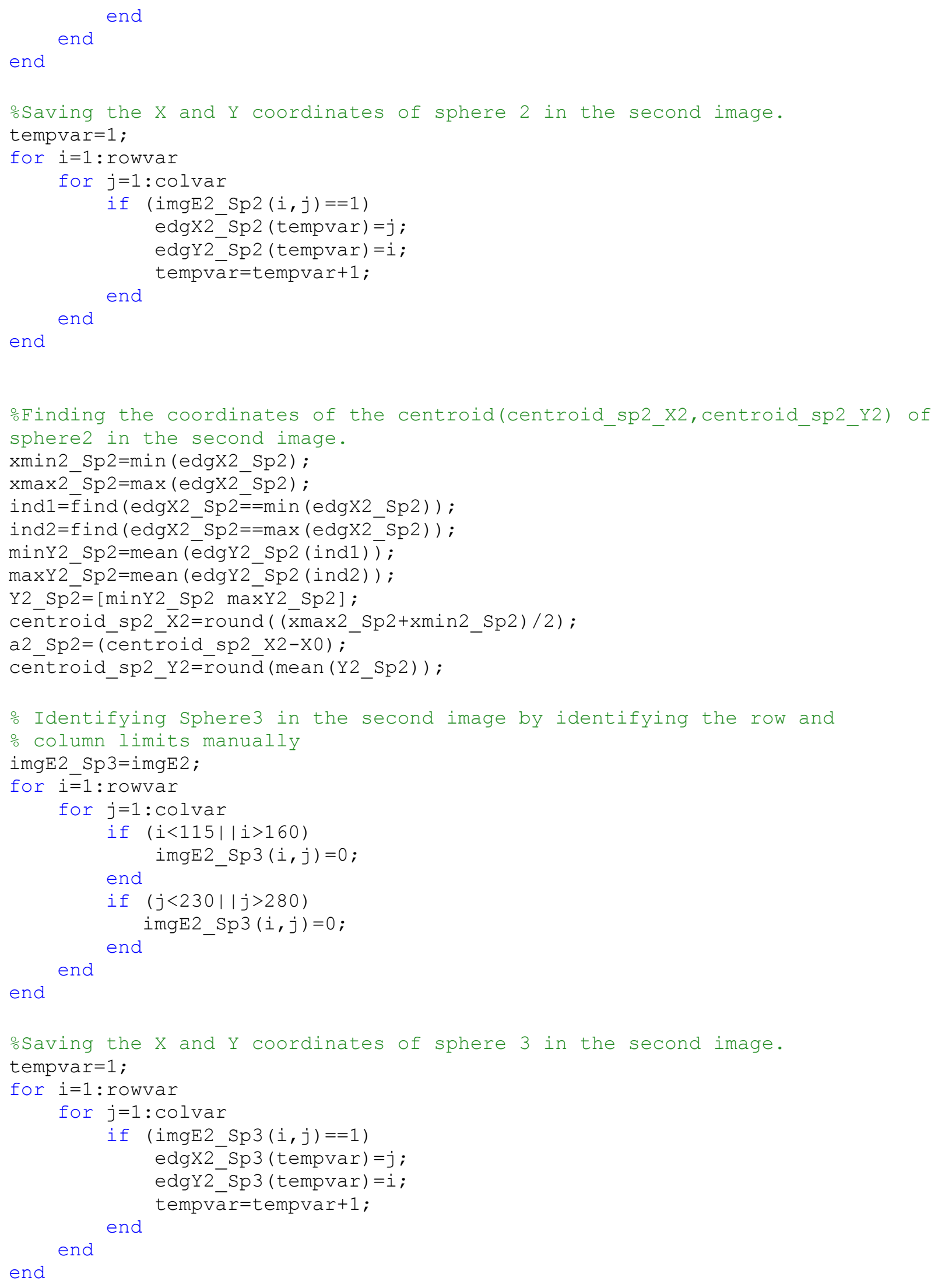




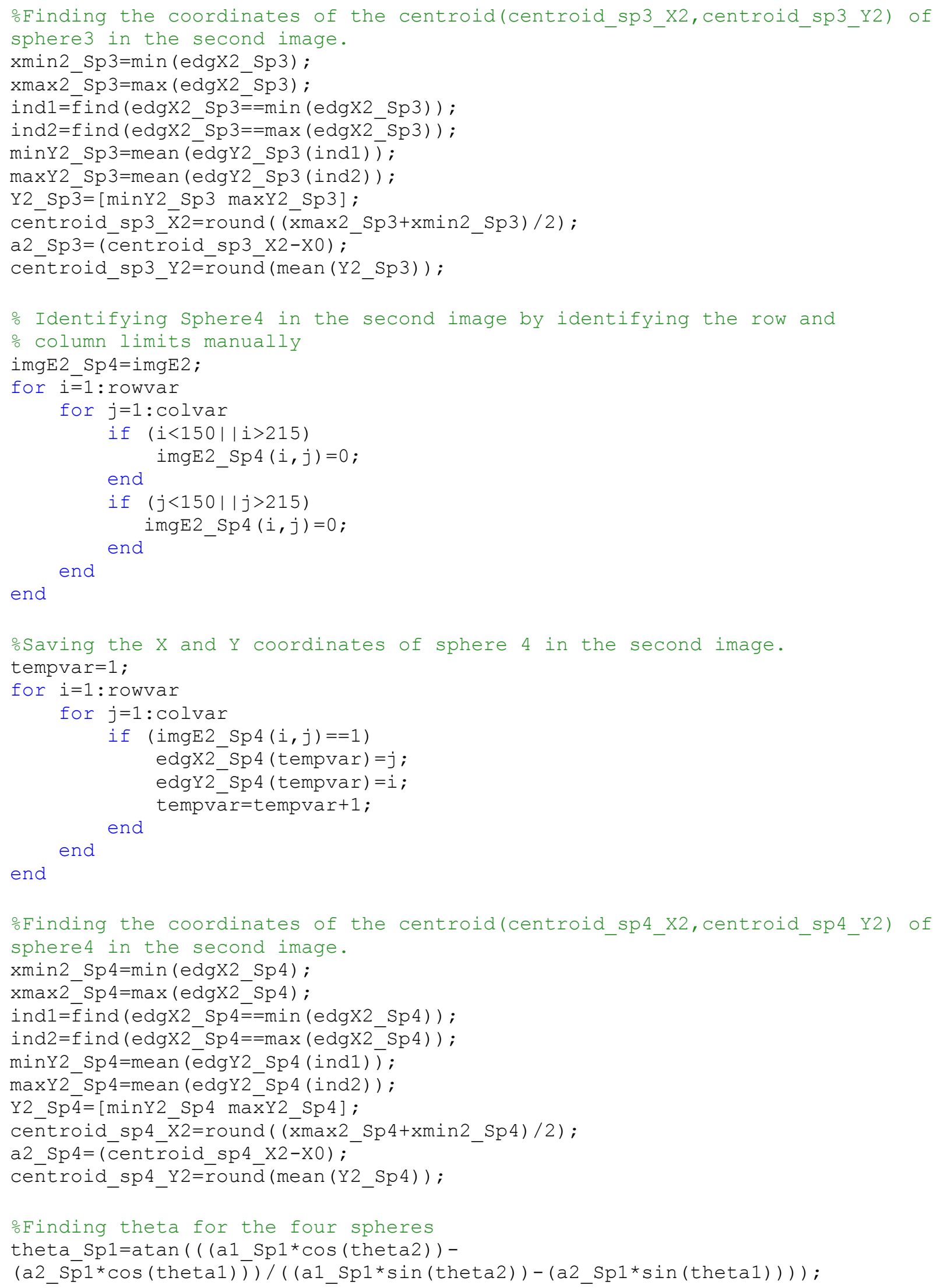




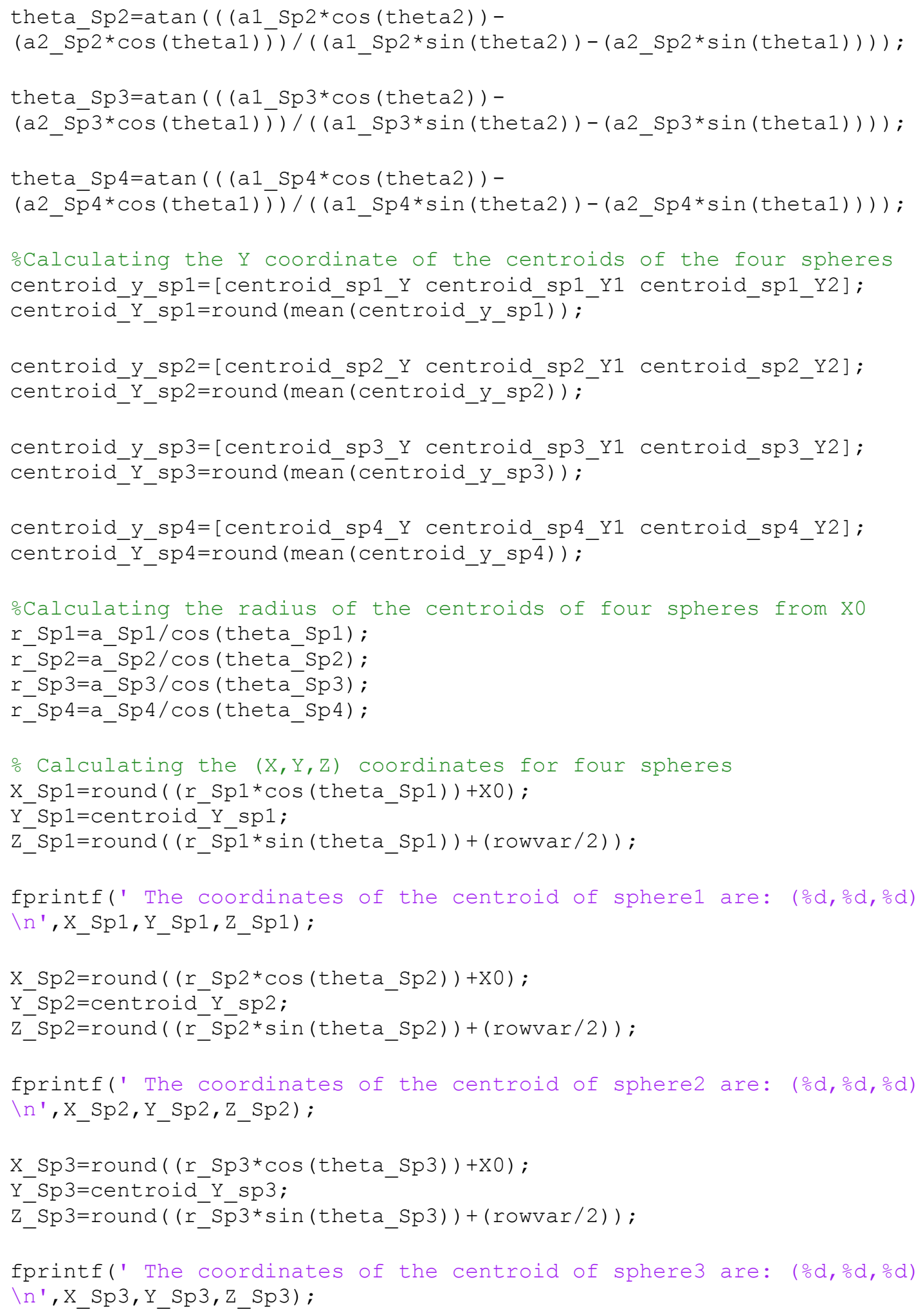


$X \_S p 4=\operatorname{round}\left(\left(r \_S p 4 * \operatorname{Cos}(\right.\right.$ theta_Sp 4$\left.\left.)\right)+X 0\right)$;

$\mathrm{Y}^{-} \mathrm{Sp} 4=$ centroid $\mathrm{Y}$ sp4;

z_sp4=round $\left(\left(r_{-}^{-} \operatorname{sp} 4 * \sin (\right.\right.$ theta_Sp4 $\left.\left.)\right)+(\operatorname{rowvar} / 2)\right)$;

fprintf(' The coordinates of the centroid of sphere4 are: ( $\left.\frac{\circ}{d}, \circ d, \circ d\right)$

$\left.\backslash \mathrm{n}^{\prime}, \mathrm{X}_{-} \mathrm{Sp} 4, \mathrm{Y}_{-} \mathrm{Sp} 4, \mathrm{Z}_{-} \mathrm{Sp} 4\right)$;

\subsubsection{Identification of the centroids in PET modality}

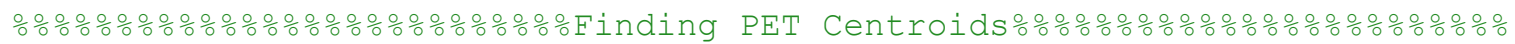
oThis program is to calculate the coordinates of the centroids of the four spheres in the calibration phantom in the PET system from the images obtained from the binary file.

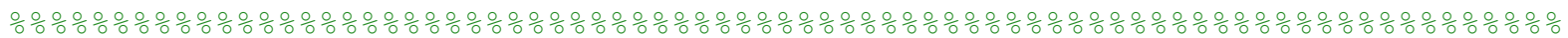

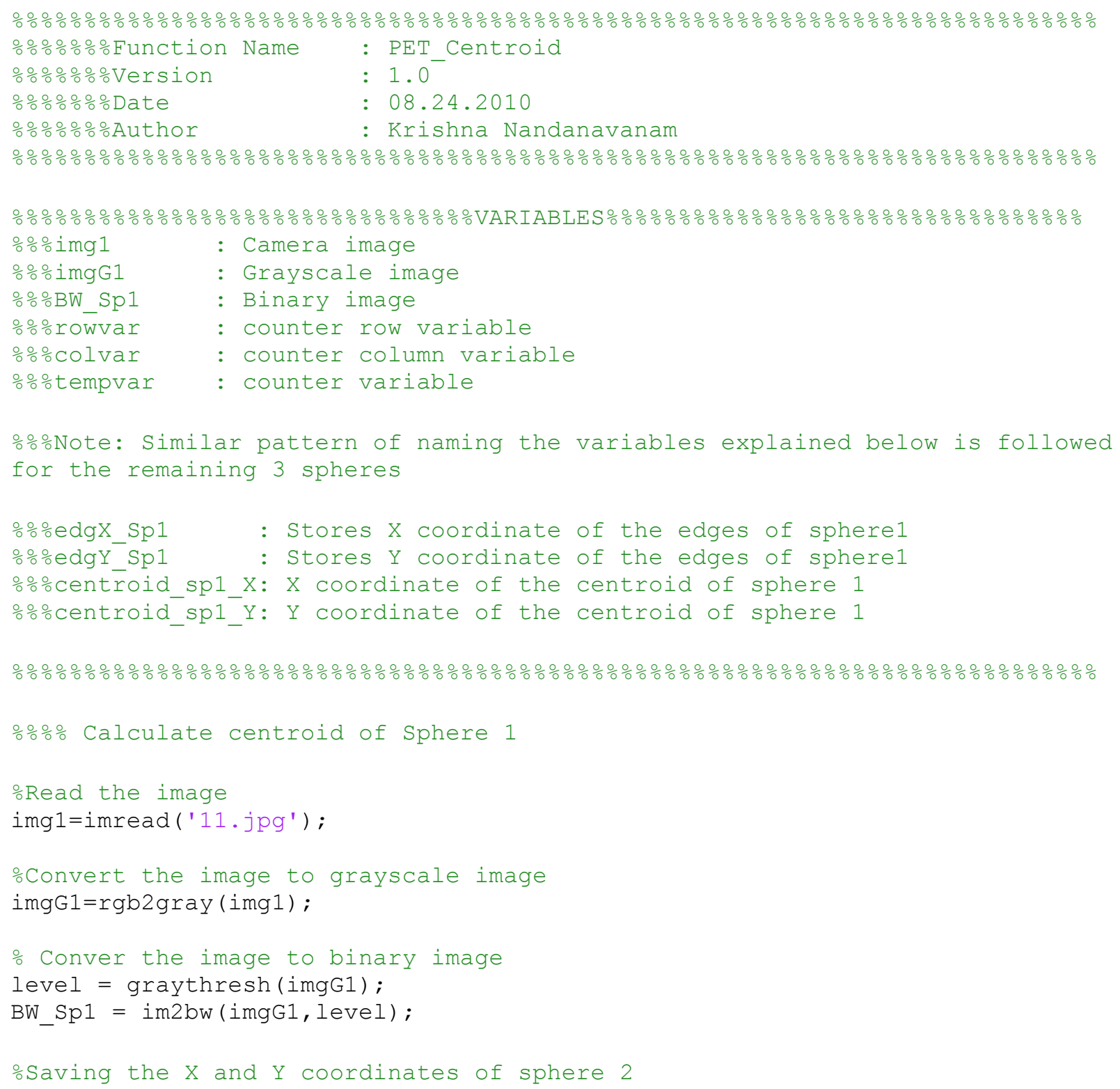




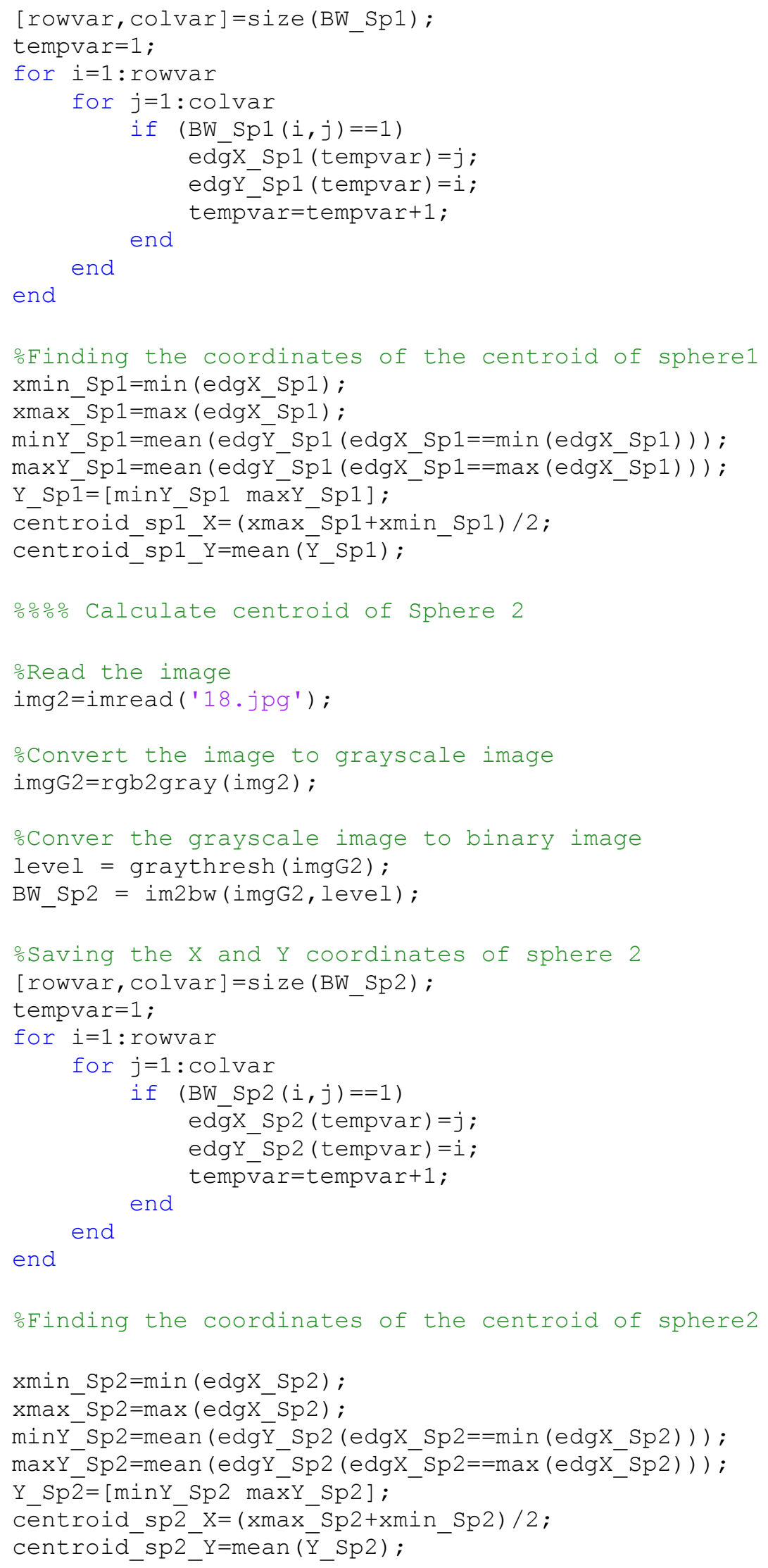




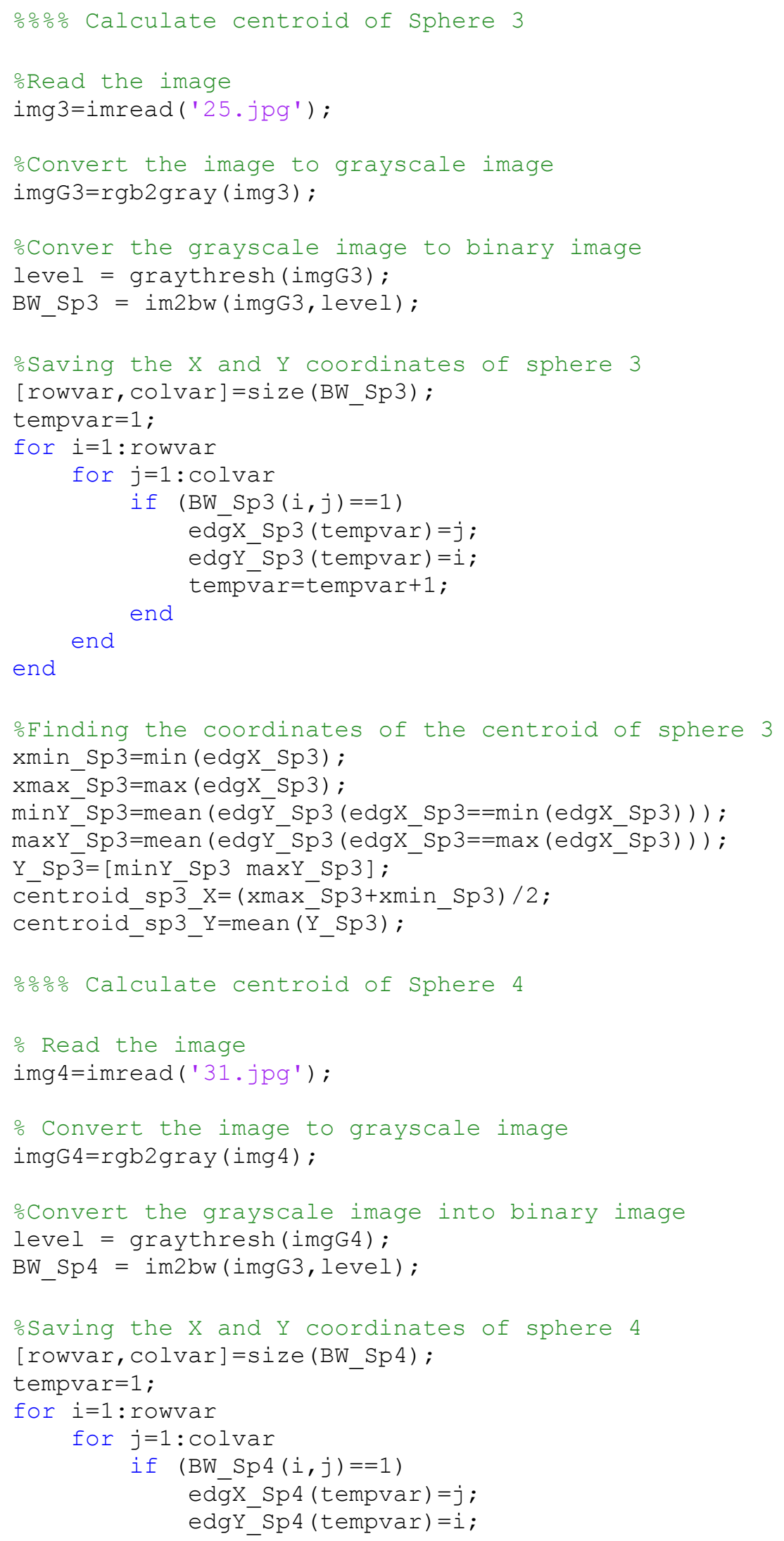




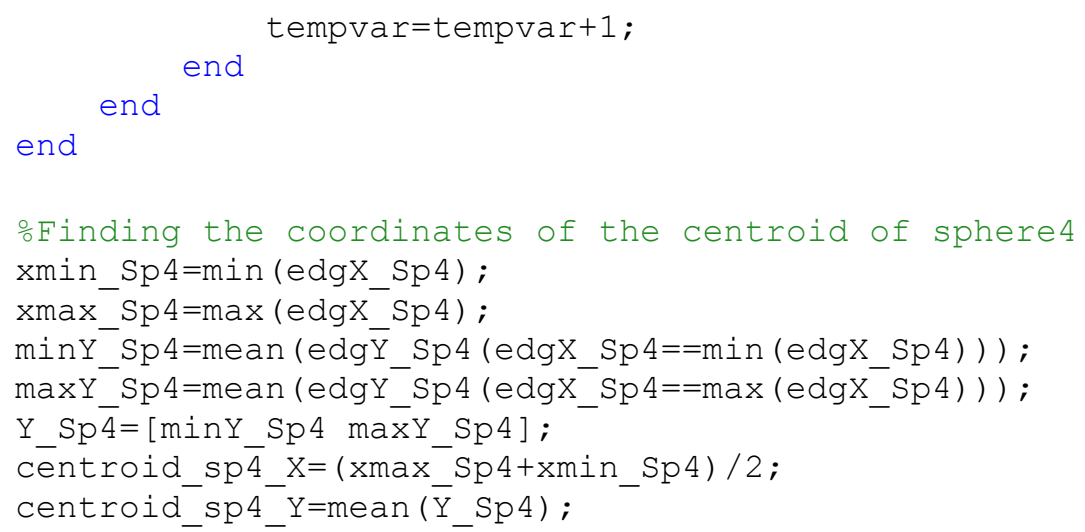

\subsubsection{Homogeneous transformation Matrix}

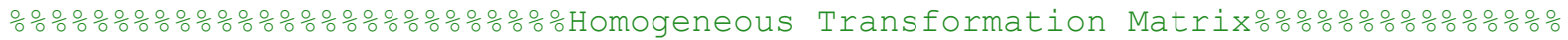
응응 This program is to calculate the homogeneous transformation matrix

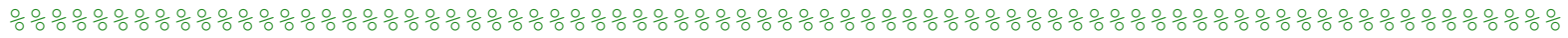

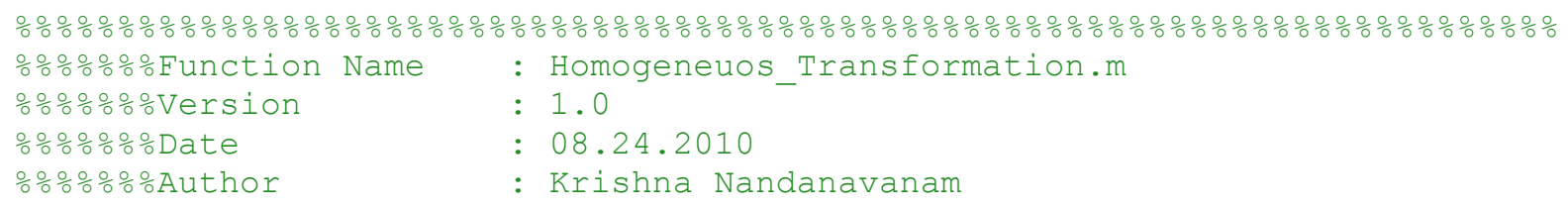


ocalculation of the transformation matrix using Gaussian elimination method $\mathrm{A} 1=\mathrm{PET} \backslash$ Optical $\mathrm{X}$;

A2 $=$ PET $\backslash$ Optical $Y$;

A3 $=$ PET $\backslash$ Optical_Z;

A4=PET\Optical_scaling;

Homogeneous_Matrix=[A1'; A2'; A3'; A4'] ; 


\subsection{Optical Slice Generation, Merging and Margin Evaluation}

\subsubsection{Function Main.m}

\% Program to read the optical camera images and generate the optical slices. Read the PET slices and merge the transformed PET slices with the respective Optical slices. Margins are evaluated for the merged slices and the images are written into a binary file MergedVolume.bin. MergedVolumefileinfo.txt has the information about the binary file.

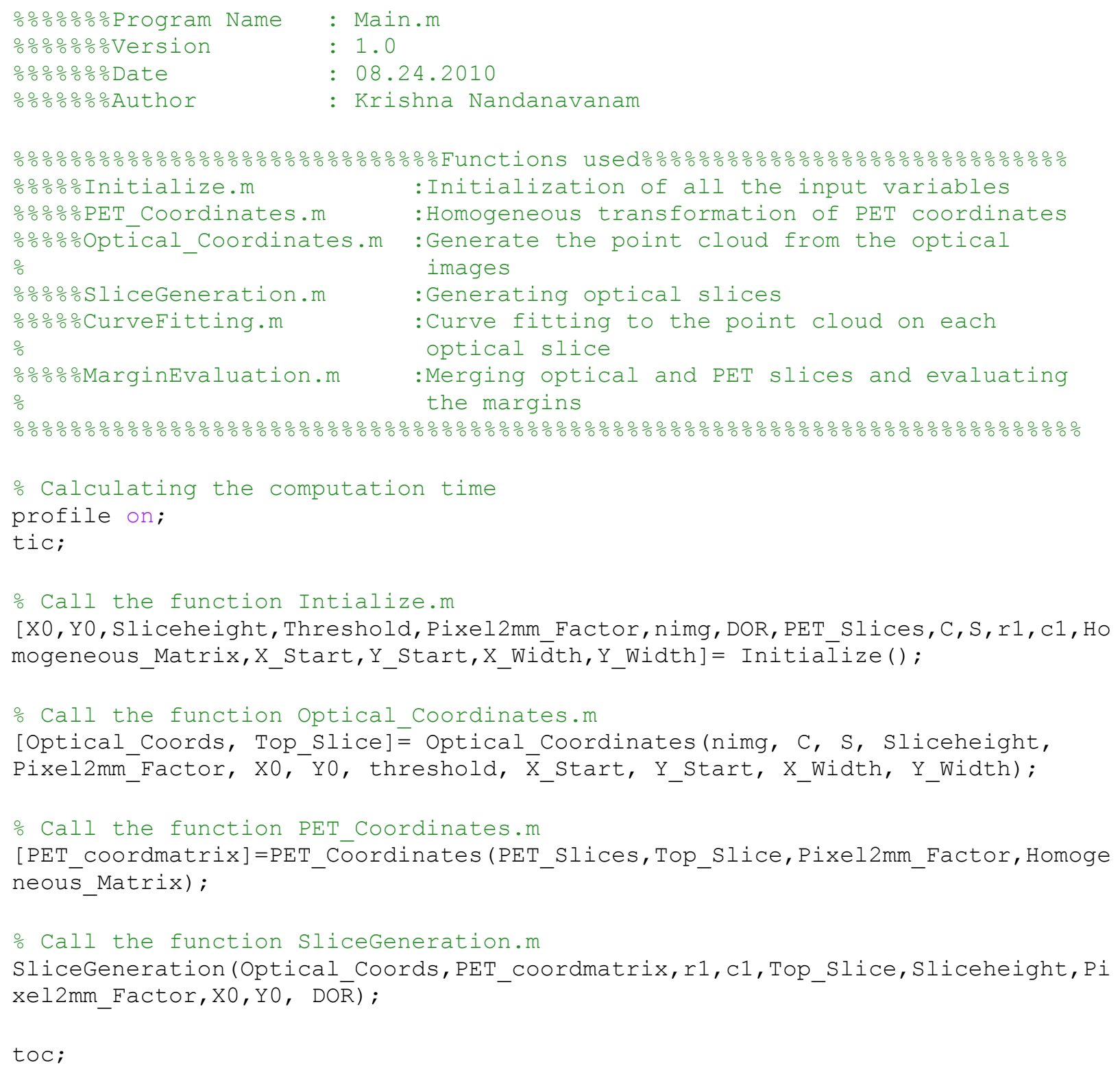




\subsubsection{Function Initialize.m}

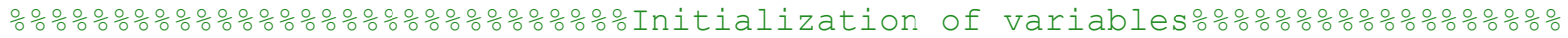
oThis function is to initialize the variables and the inputs are to be given by the end user.

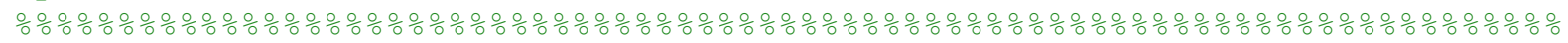

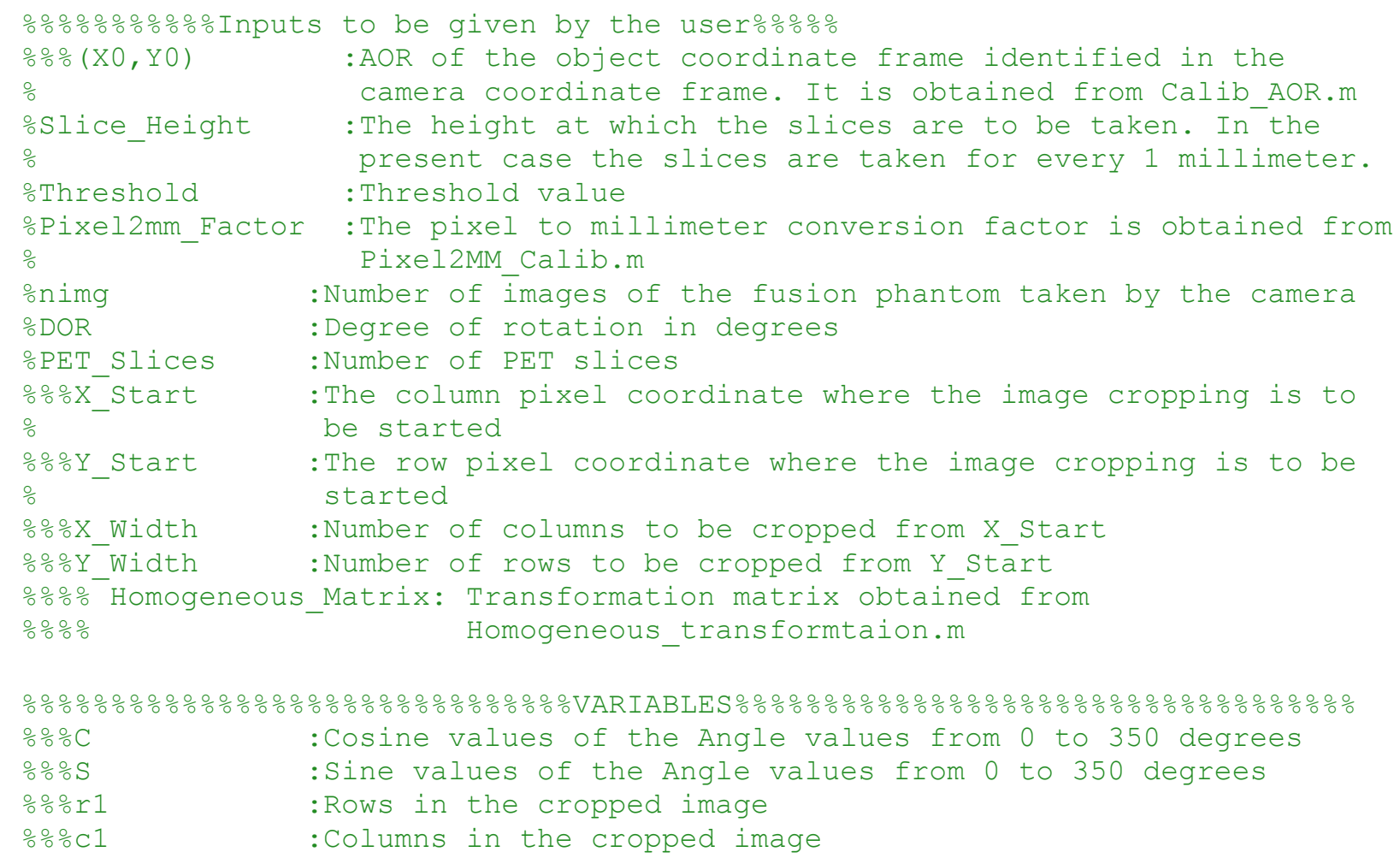




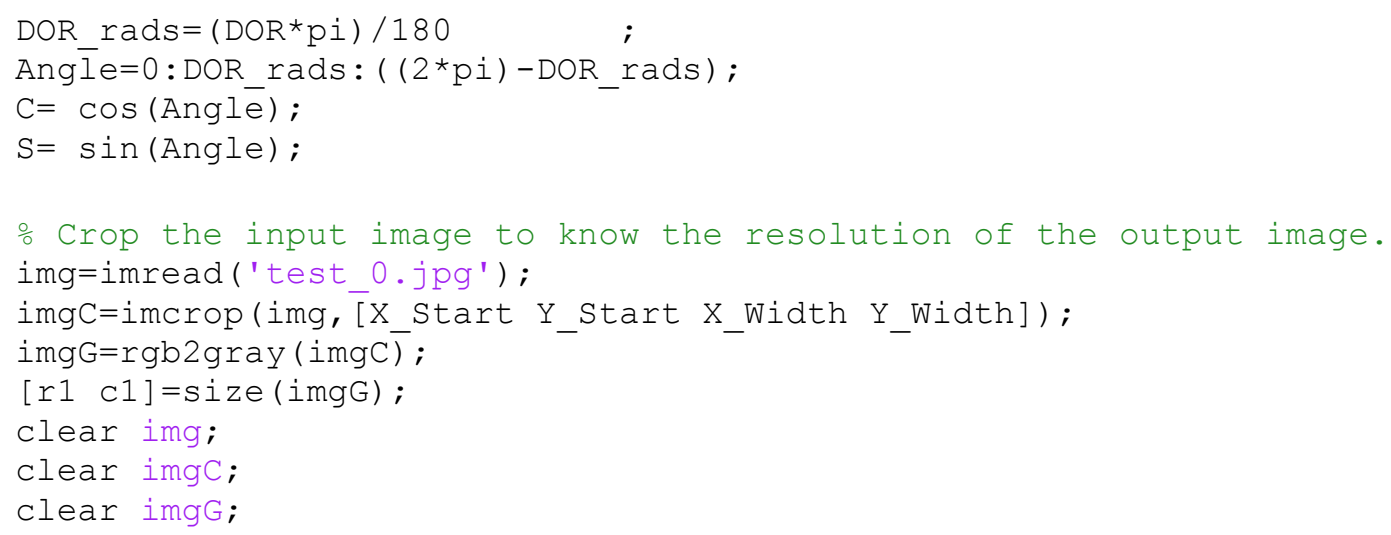




\subsubsection{Function Optical_Coordinates.m}

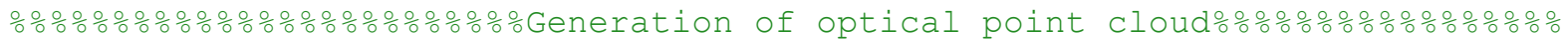

\% This function is to generate the point cloud of the optical system using

\% the camera images as input.

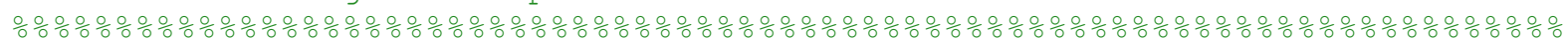

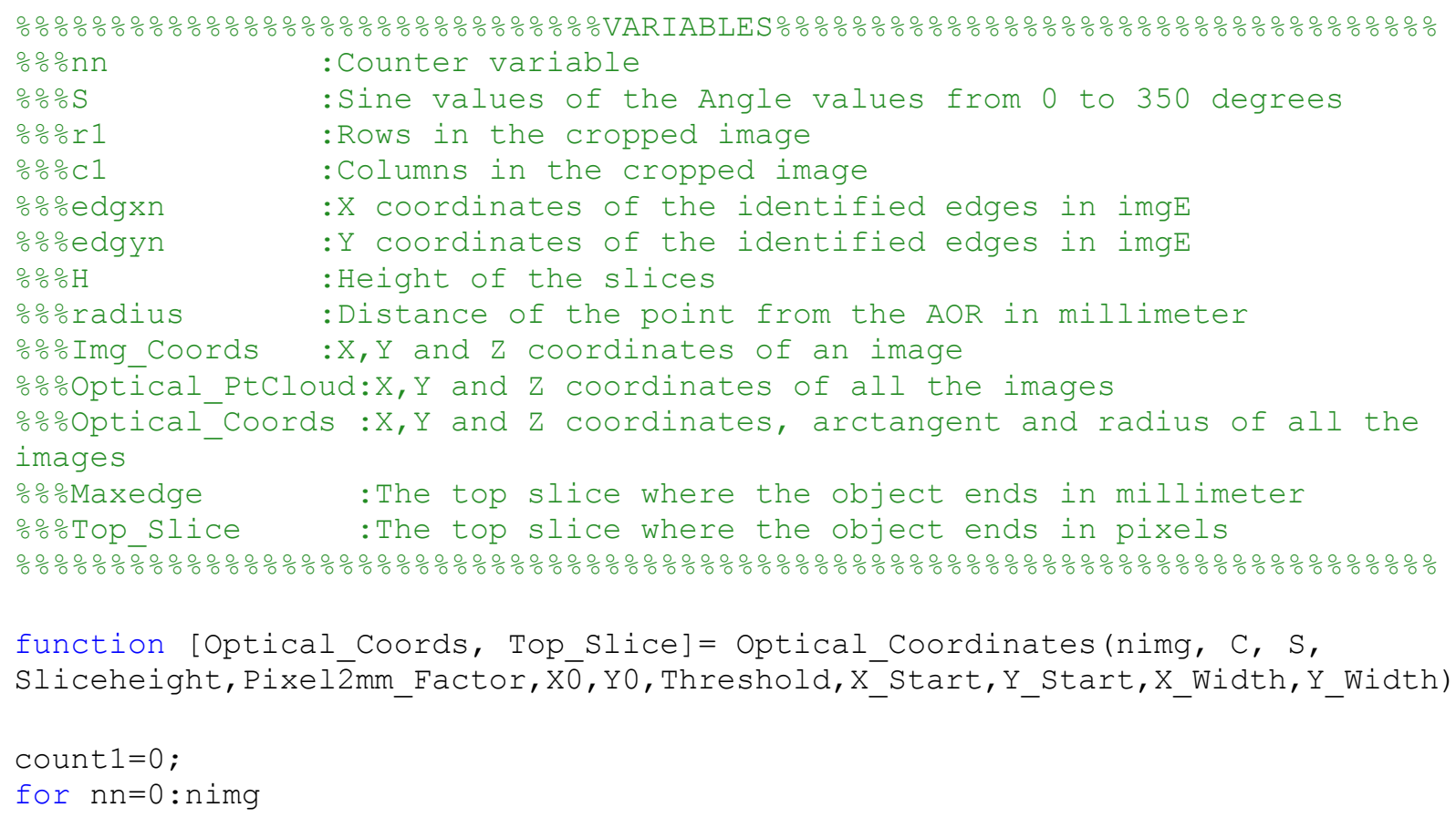




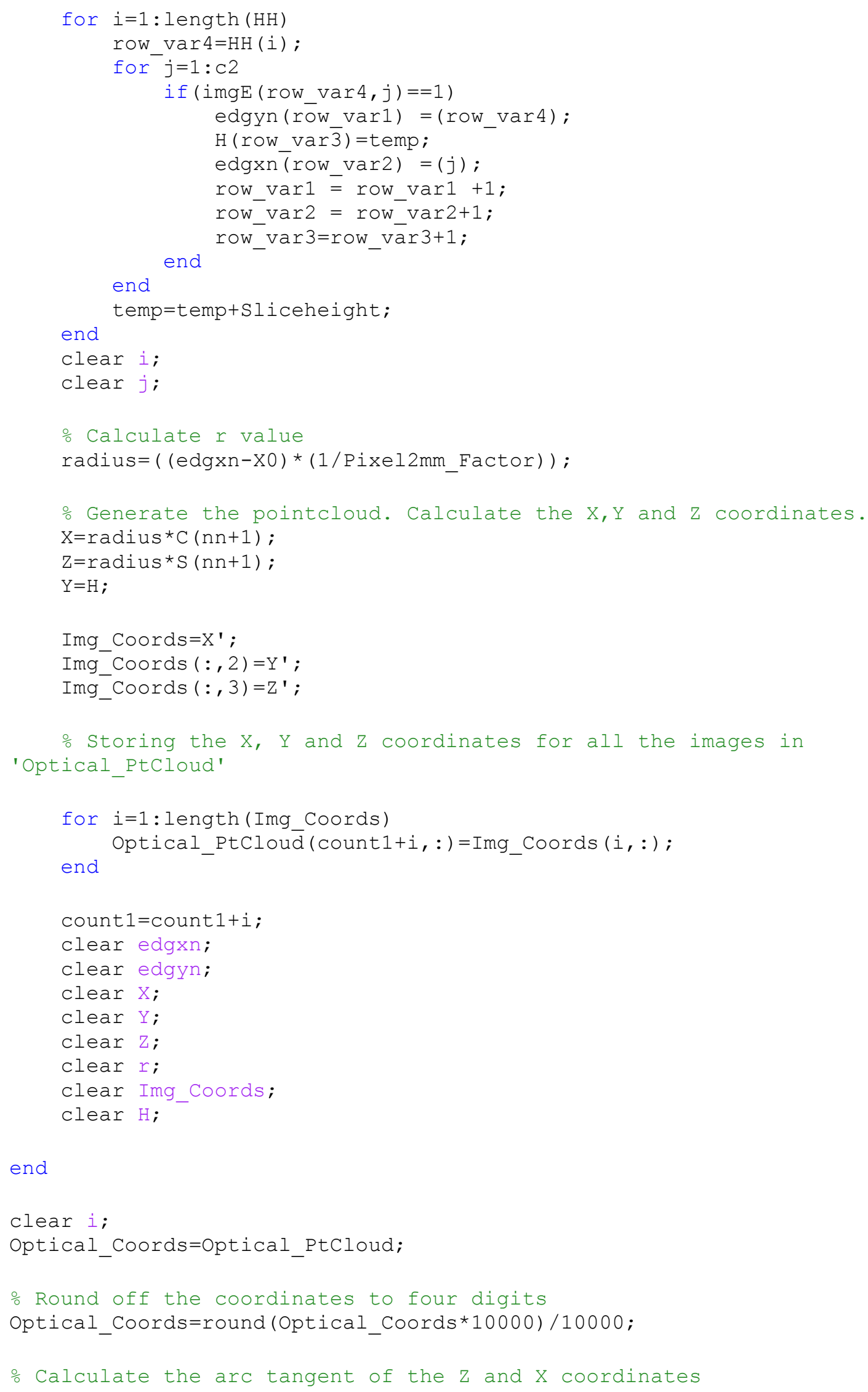




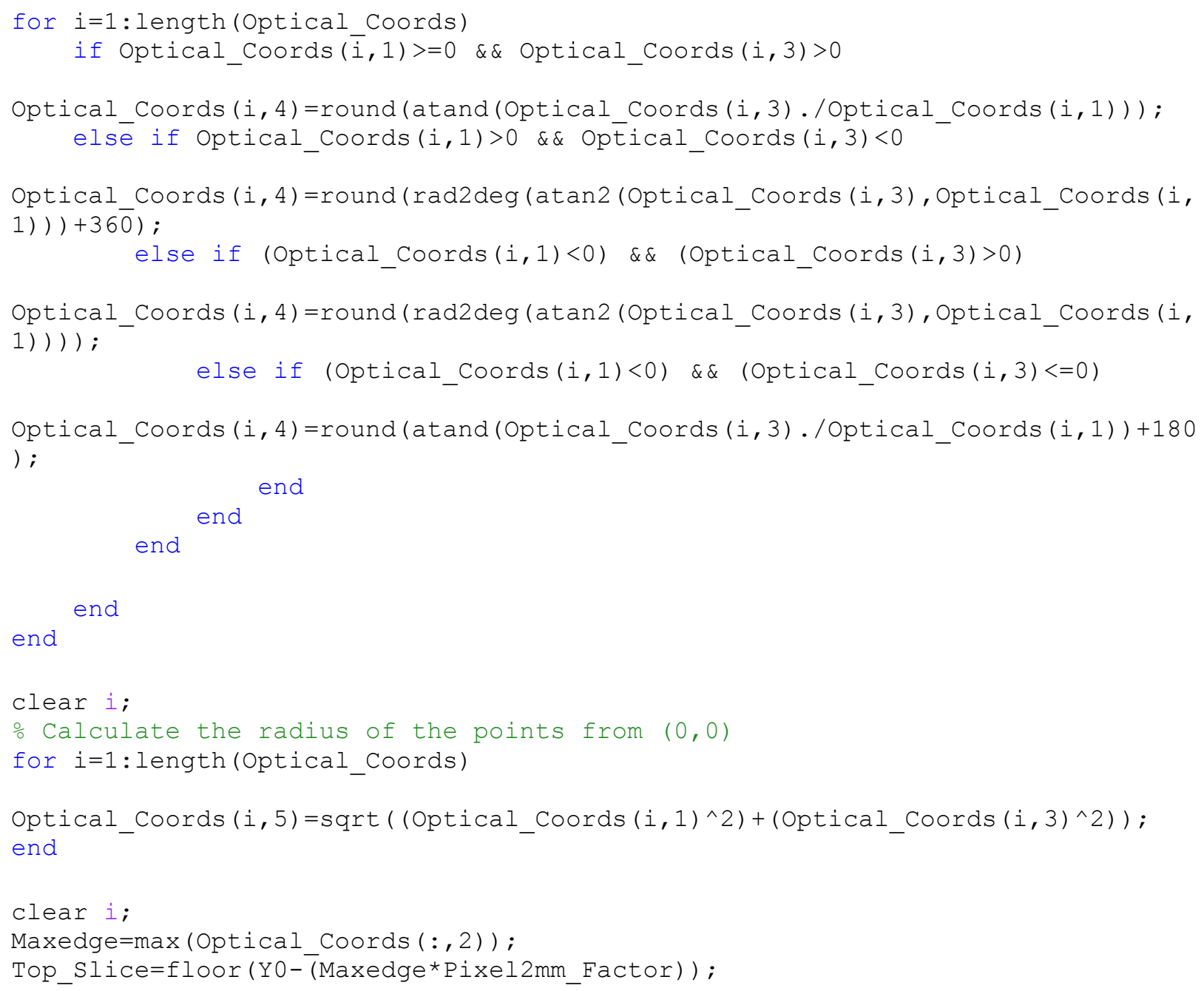




\subsubsection{Function Pet_Coordinates.m}

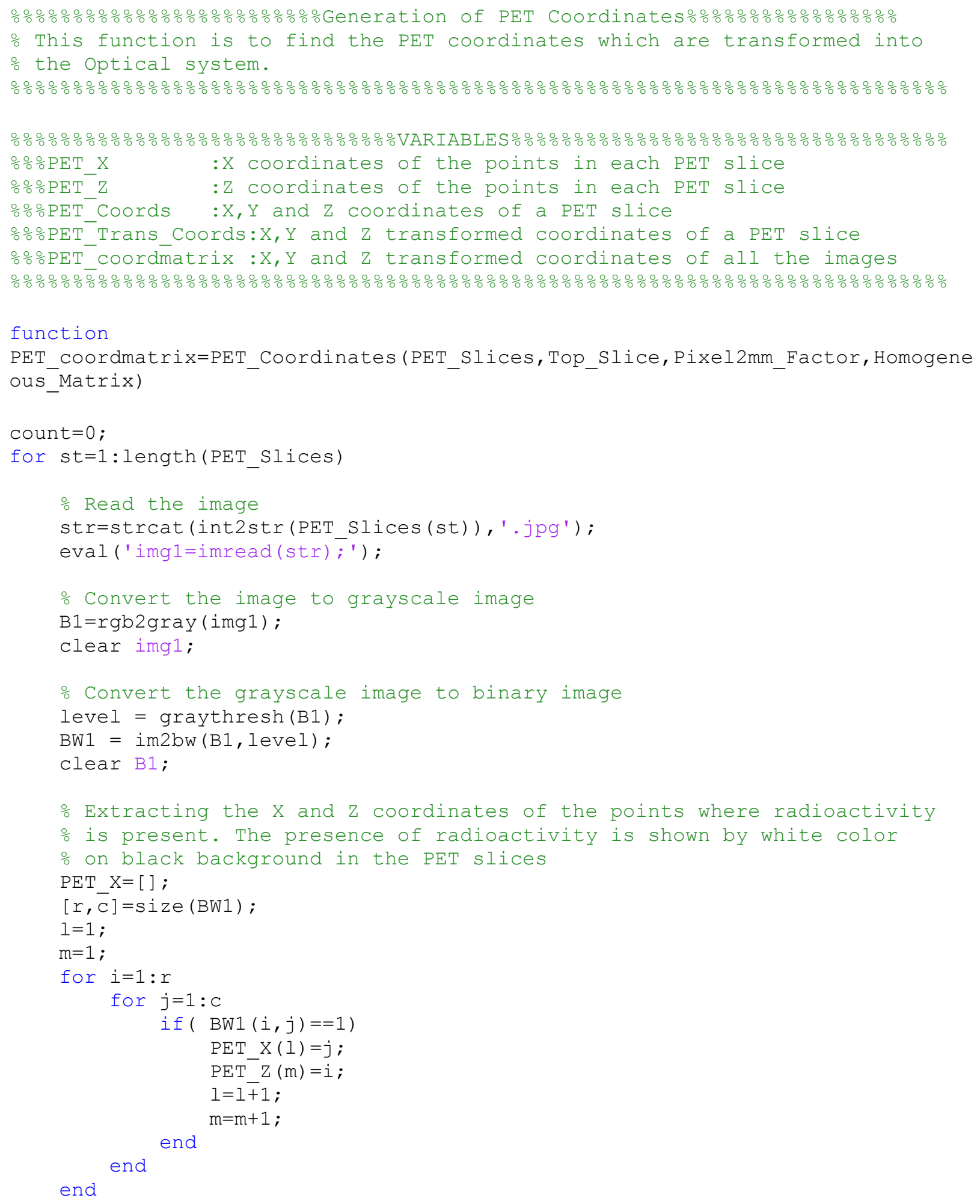




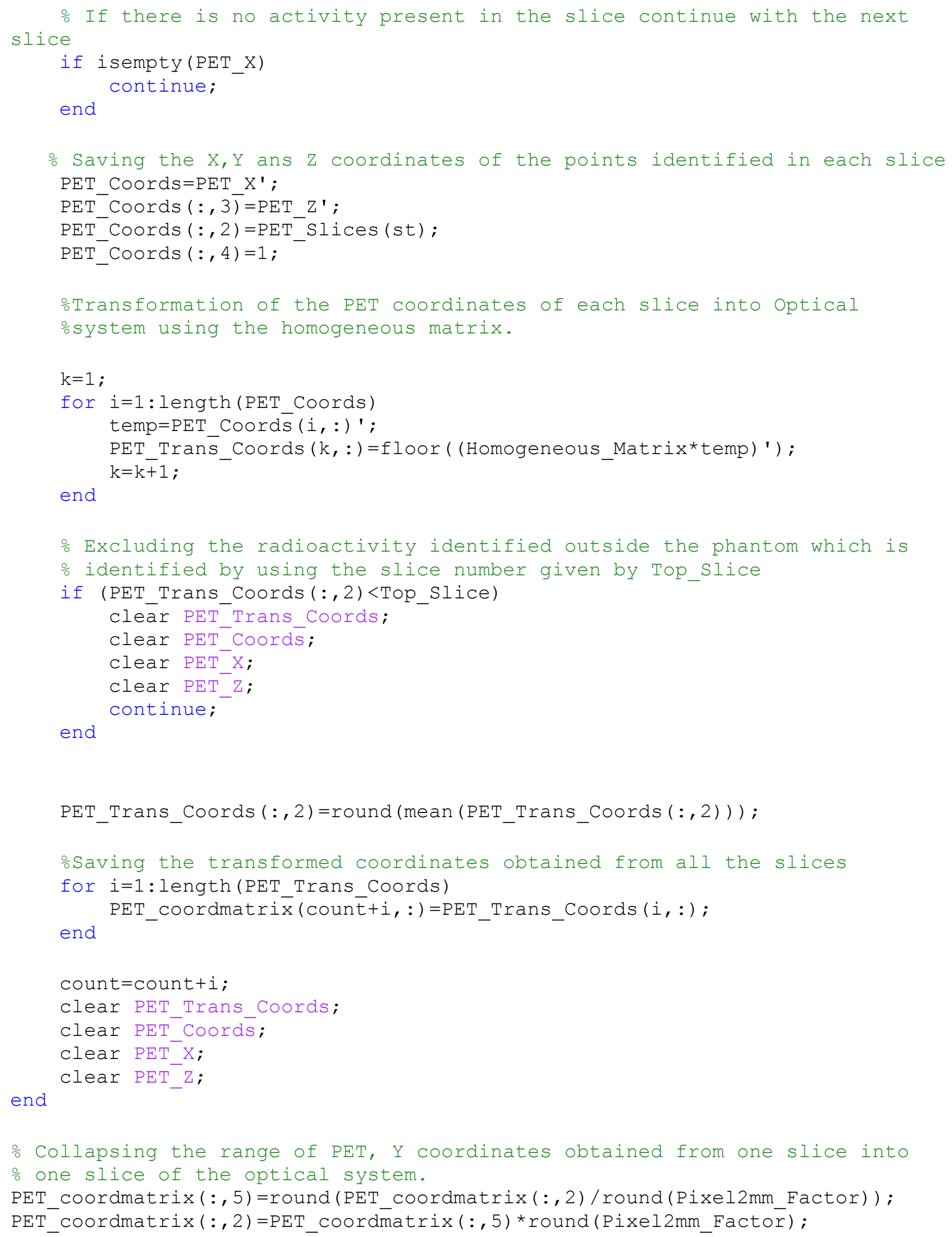




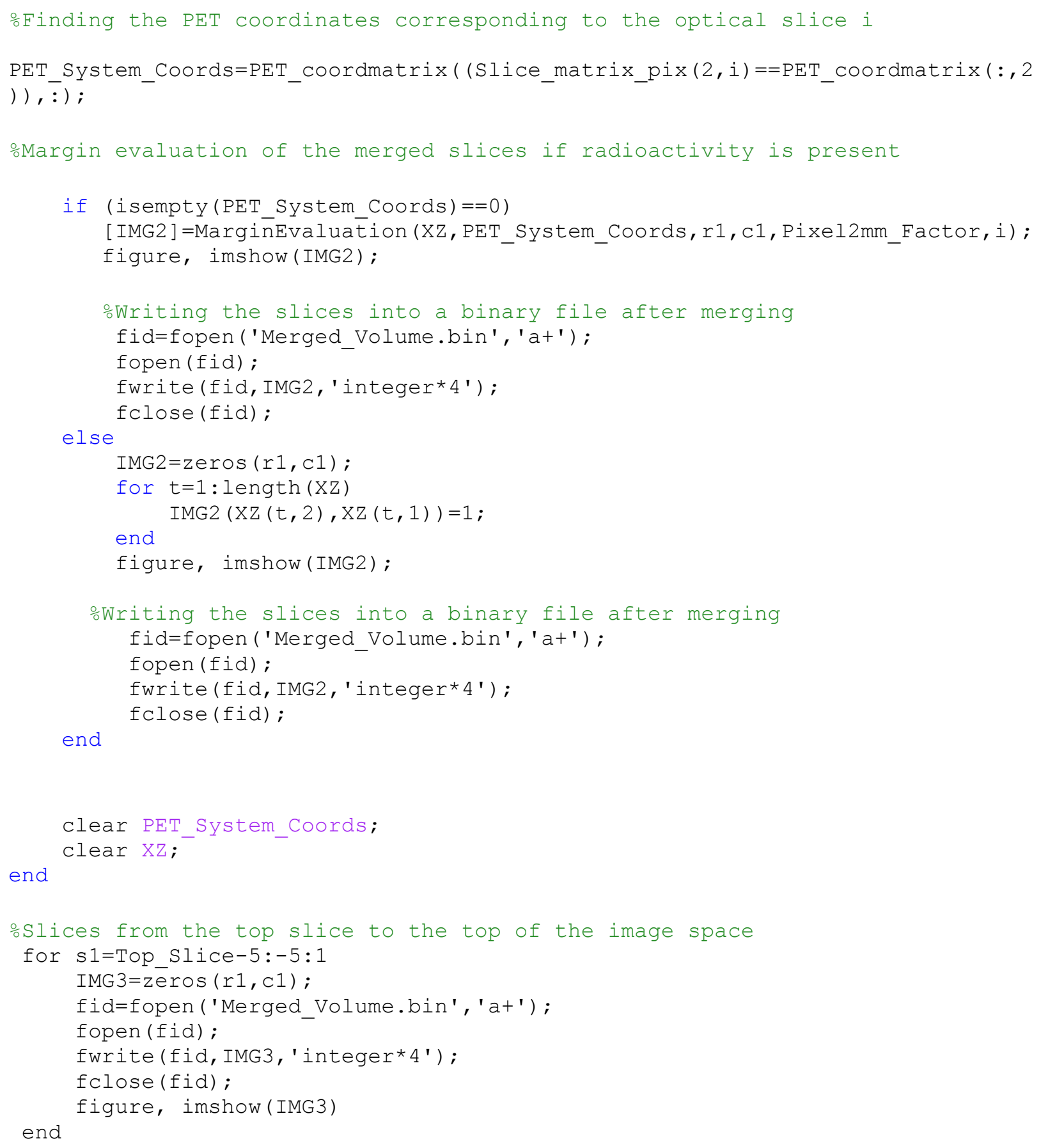




\subsubsection{Function CurveFitting.m}

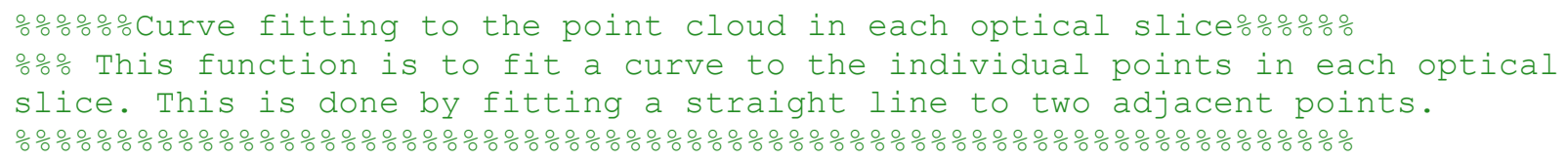




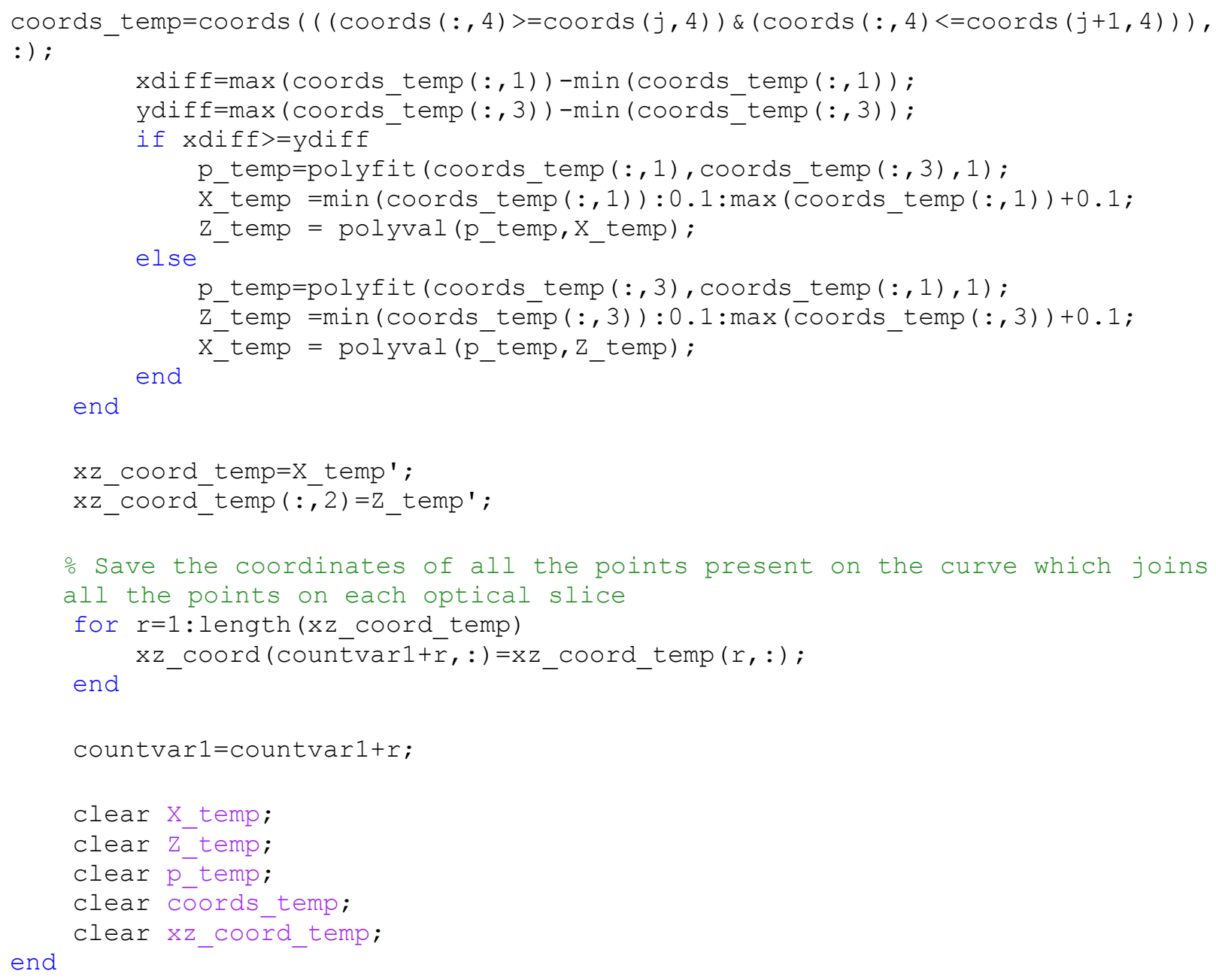




\subsubsection{Function MarginEvaluation.m}

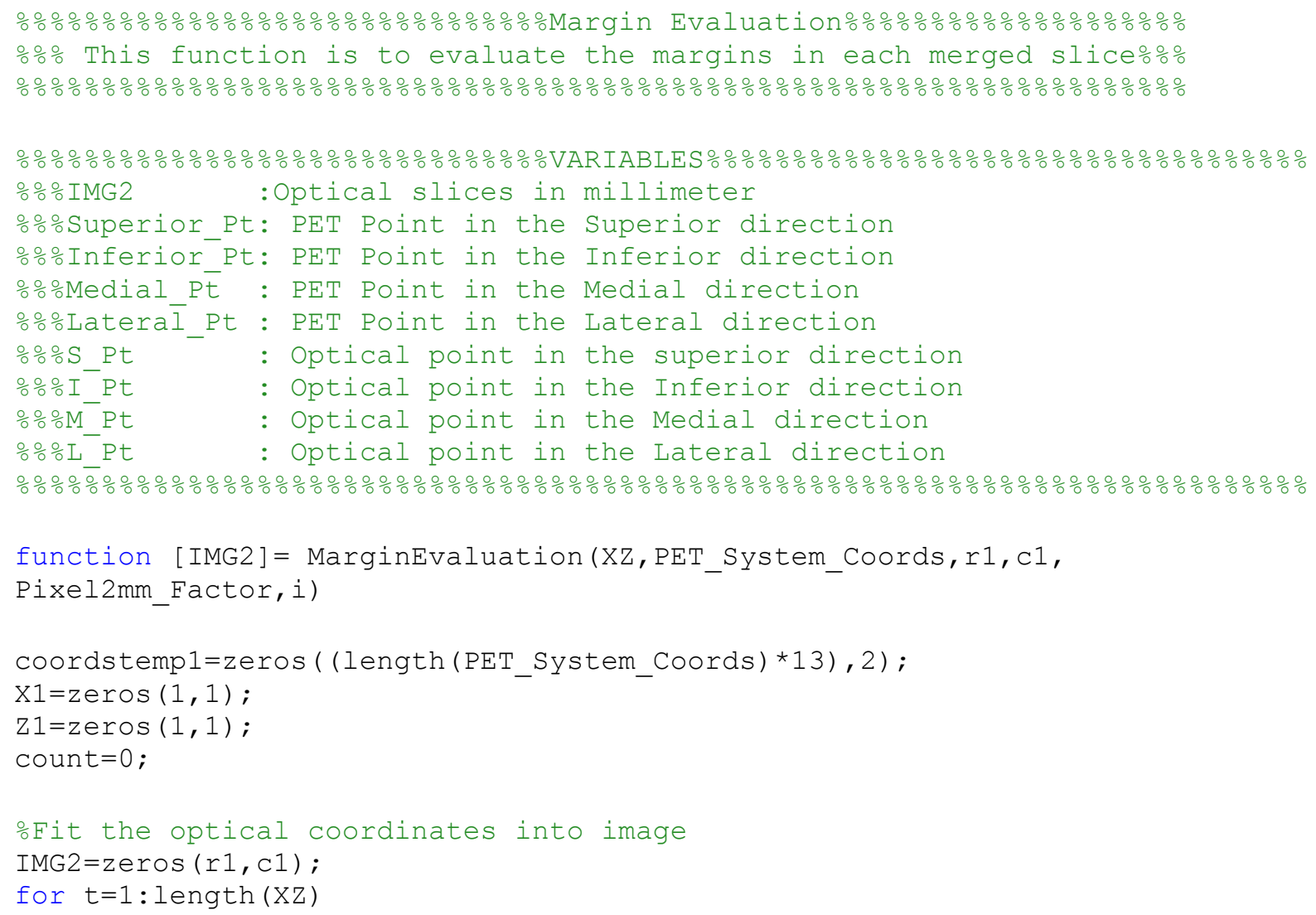




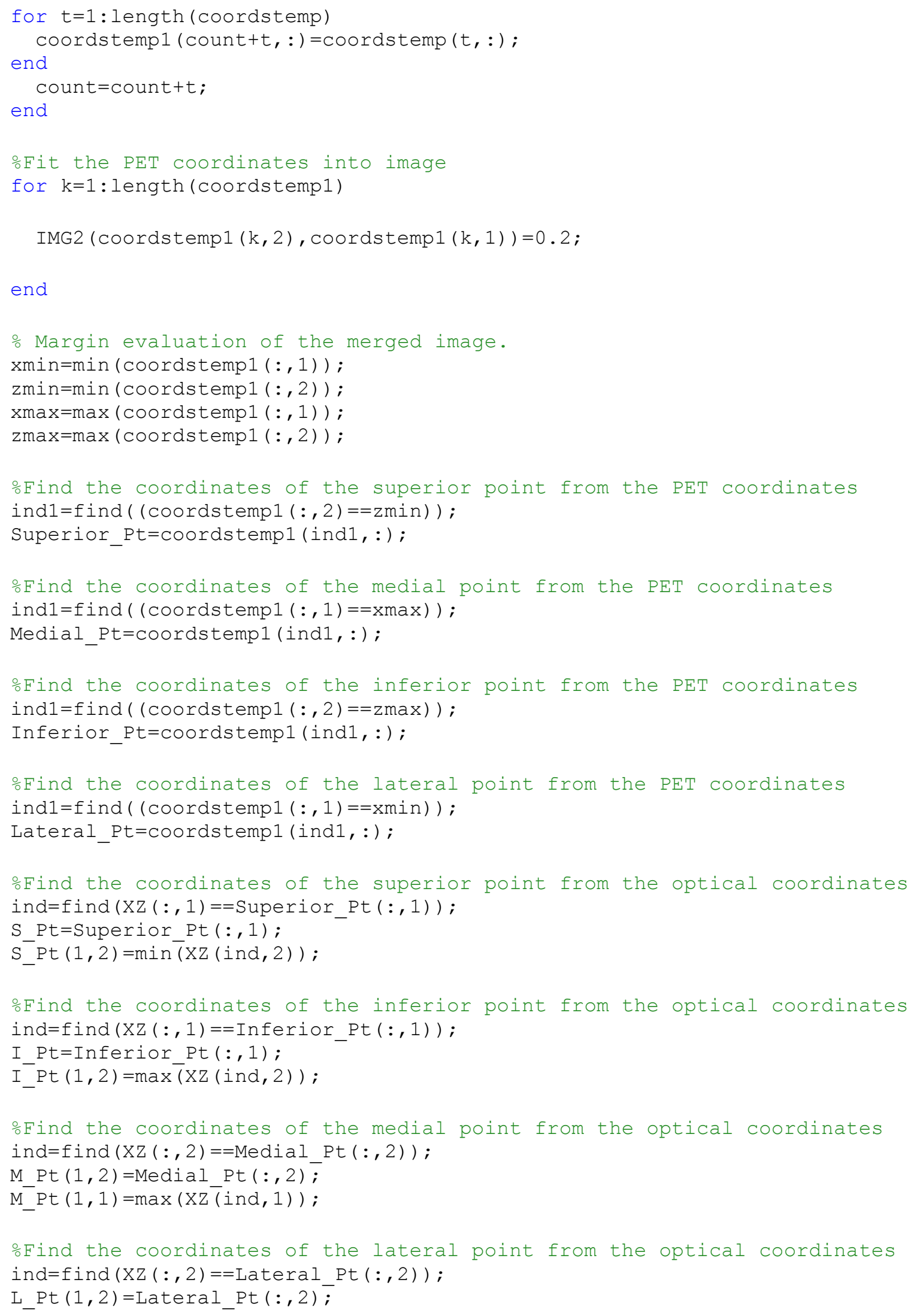




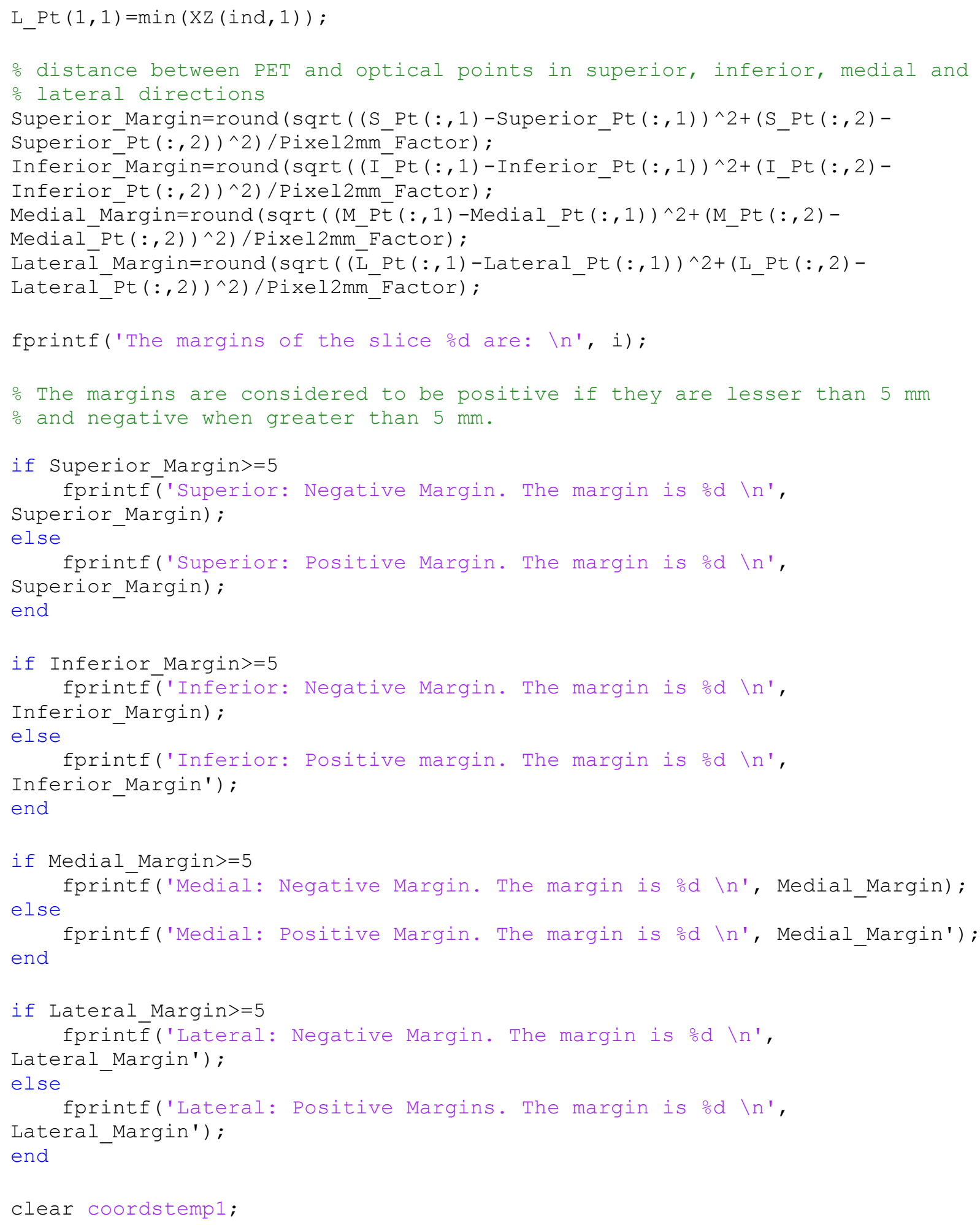

